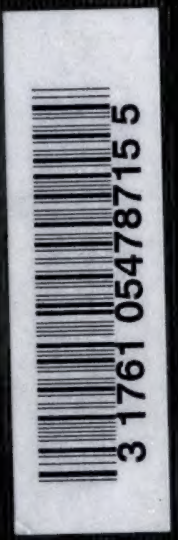


MAR \& 1914

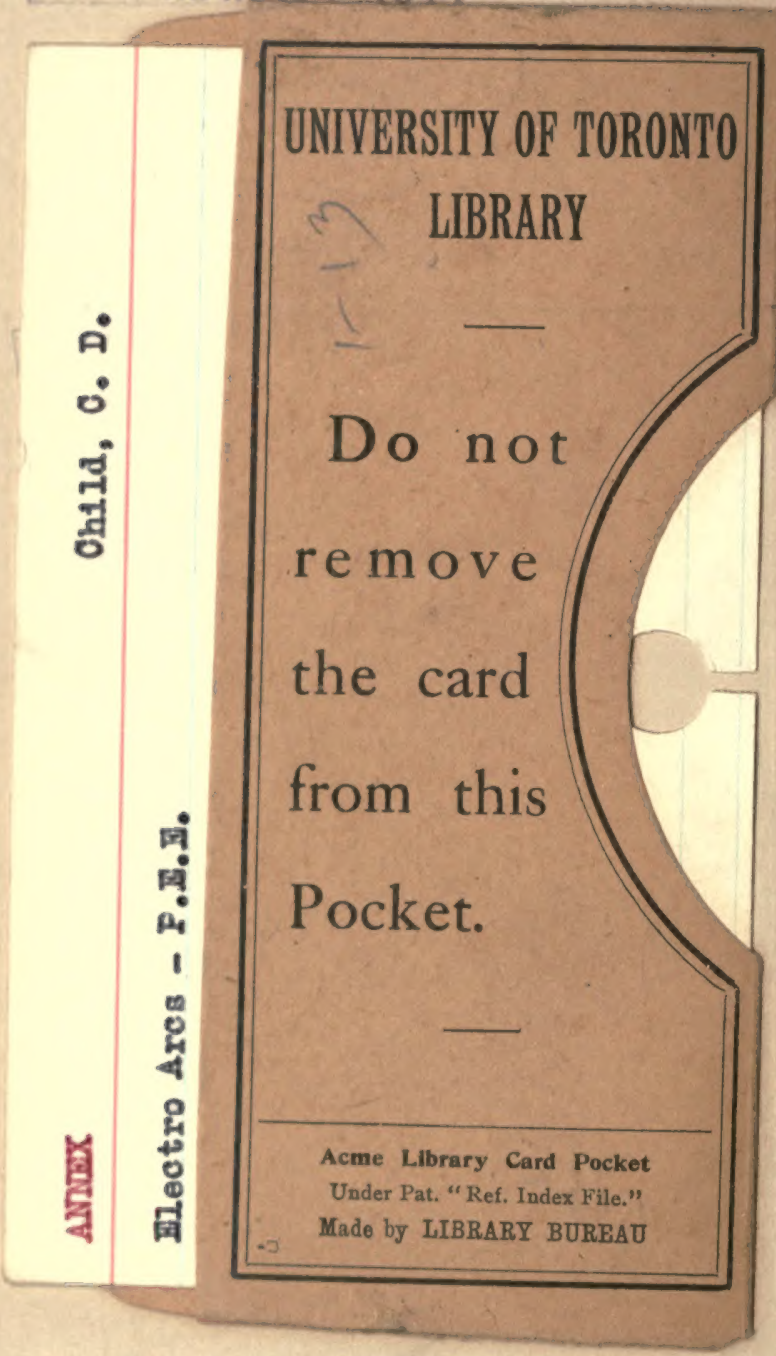


The D: Van Nostrand Company intend this book to be sold to the Public at the advertised price, and supply it to the Trade on terms which will not allow of reduction. 

Digitized by the Internet Archive in 2007 with funding from Microsoft Corporation 


\title{
ELECTRIC ARCS
}

EXPERIMENTS UPON ARCS BETWEEN DIFFERENT ELECTRODES IN VARIOUS ENVIRONMENTS AND THEIR EXPLANATION

\author{
BY \\ CLEMENT D. CHILD, Pн. D.
}

Professor of Physics at Colgate University

58 ILLUSTRATIONS

\author{
NEW YORK \\ D. VAN NOSTRAND COMPANY \\ 25 PARK PLACE \\ I9I 3
}


Copyright, I9I3,

BY

D. VAN NOSTRAND COMPANY

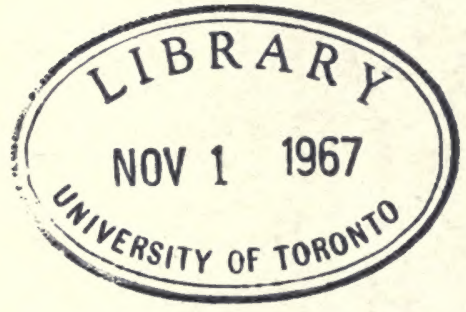

Stanbope Ipress

F. H. GILSON COMPAN $Y$ BOSTON, U.S.A. 


\section{PREFACE.}

While the electric arc is one of the most common things of modern life, an understanding of it is not common. This is largely due to the difficulty of becoming familiar with the investigations which have been made on this subject. There have indeed been many articles published concerning it, but they are scattered through many publications, and the data given by different experimenters are not consistent with each other, and none of the explanations are entirely satisfactory.

"The Electric Arc," by Mrs. Hertha Ayrton, gave an account of the experiments which were performed prior to I898, but very little investigation had been made at that time of any arc except the open carbon arc, and none of the explanations based on the ionic theory had been given, so that by far the greater part of what is now known concerning the arc could not then be described. An excellent work in German, "Der elektrische Lichtbogen" by Berthold Monash, has more recently appeared, but this deals but briefly with the theoretical side and does not meet the requirements of those who would read English rather than German.

It, therefore, seemed desirable to offer another discussion of the electric arc, giving especial attention to the explanation of the phenomena and to those investigations which have been made since the publication of Mrs. 
Ayrton's book. A rather full account has, accordingly, been given to such experiments as those on the mercury arc, and to a discussion of the more recent theories.

The use of the arc in commercial ways has already been ably discussed in such books as those on Electric Lighting, Photometry and Wireless Telephony. Accordingly, these topics have received less attention here.

A few pages, however, are given to photometry and to the whistling arc, since these are of interest from a scientific as well as from an industrial standpoint. No account has been given concerning the use of the arc in chemical and metallurgical processes, since its function there appears to be merely to produce a high temperature and a study of these phenomena would give us no knowledge concerning the arc itself.

I have endeavored to keep in mind the needs of those who may wish to make investigations in the future. An effort has, therefore, been made to give references to all the important articles on this subject, excepting those which relate only to the commercial side or to those concerning investigations in which the arc was merely a means for studying some other phenomenon, as when used to produce the spectrum of a metal.

It would often have made much simpler and more satisfactory reading; if I could have given a brief and definite statement of the laws governing the action of the arc, instead of producing so extended a review of what different experimenters have thought about these laws, but in the majority of cases it is not yet known what the laws are and the only available method is to discuss the results of those who have endeavored to find them. Not only is there this uncertainty concerning the laws, but the expla- 
nations often raise more questions than they settle. This, however, is not an unusual condition and the explanations may, at least, serve in helping others to make complete that which is lacking.

HAMTLTON, N.Y.

Clement D. Child.

Oct., I9ז2. 



\section{TABLE OF CONTENTS.}

CHAPTER PAGE

I. Introduction $\ldots \ldots \ldots \ldots \ldots \ldots \ldots \ldots \ldots \ldots \ldots$ I

Definition of Electric Arc................... I

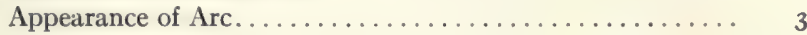

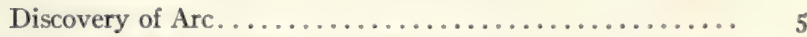

II. Arc in Air Between Carbon Electrodes ............ 8

Relation Between Potential Difference, Current and Length

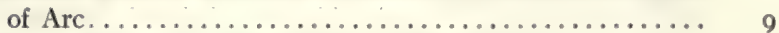

E.M.F. Required as Distinct from Voltage of Arc . . . . . . I6

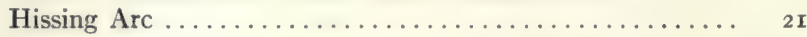

Fall of Potential in Different Parts of Arc.......... 22

Counter E.M.F. of Arc....................... 25

Meaning of "Resistance of Arc"................ ${ }_{2} 8$

"Negative Resistance".................... 3I

"Forward E.M.F." ....................... 33

Residual E.M.F ..................... 35

Temperature of Arc .................... 40

Variation in the Temperature of the Arc........... 44

Effect Produced by Cooling Electrodes............. 46

Size of Anode Crater...................... 48

Enclosed Arc............................. 49

Miscellaneous......................... 50

III. Arc in Air Between Other Substances than Carbon... 53

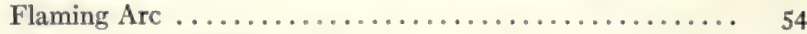

Arc Between Metals...................... 6r

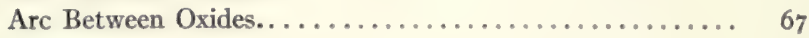

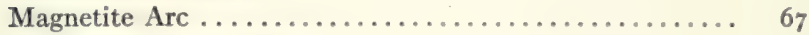

Arc Between Electrolytes................... 69

Arc Between Unlike Electrodes................. 69

IV. Arc with Pressures Greater or Less than Atmospheric

Pressure and in Other Gases than Atr......... 73

Arc with Pressures Greater than One Atmosphere....... 73

Arc with Pressures Less than One Atmosphere......... 74

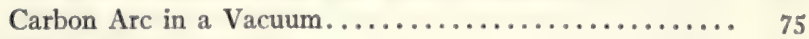

Metal Arc in a Vacuum .................... 8r

Arc in Other Gases than Air................... 83 
V. Mercury Arc............................... $8_{7}$

Temperature............................. gr

Characteristic Curves....................... 93

Cathode Drop............................. ${ }_{96}^{96}$

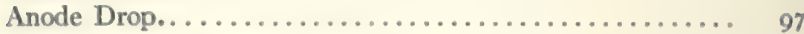

Electric Force Through Arc.................. 97

Arc in Quartz Tubes......................... 100

Velocity of Ions ........................ ror

Modification of Arc to Produce White Light.......... I02

VI. Alternating Current Arc.................... 105

Between Carbons......................... 105

Characteristic Curves....................... Iо

Current and Potential Difference at Different Phases..... Iо 107

"Dynamical" Characteristic Curves................. r ro

Fall of Potential in A.C. Arc.................. II2

Phase Difference in Arc..................... $\mathbf{I I 3}$

Arc Between Metals...................... II4

Arc Between Unlike Electrodes.................. II8

Arc in Other Gases than Air................. IIg

Mercury Arc Rectifier....................... I 20

VII. Рнотомetry of the Electric ARC.............. 126

Distribution of Light..................... I 28

Effect on Candle Power of Length of Arc, Current, Etc.... I30

Intrinsic Brightness of Crater ................ 132

Flaming Arcs........................... $\mathbf{r}_{32}$

Metal, Including Mercury, Arcs............... 134

Alternating-current Arcs.................... 135

Comparison of Different Kinds of Arcs............ I39

Spectrum of Arc ....................... 142

ViII. Use of ArC in Wireless Telephony............. I 46

Whispering Arc........................ 146

Whistling Arc............................ I48

Application of Whistling Arc to Wireless Telephony...... ${ }^{153}$

IX. Theory of the Electric ARc.................. ${ }_{15} 6$

Definition of Ions and Electrons................. ${ }_{15} 6$

Causes Producing Ions...................... ${ }_{15}^{8}$

Ionization by Impact...................... ${ }_{15} 5^{8}$

Ionization by Hot Solids . . . .................. 159

Fall of Potential Through Arc................. I60

Ionization at Surface of Cathode with Discharge at low

Pressure.................................. I6I

Ionization at Surface of Anode.................. 162 
IX. Ionization at Cathode of Arc................. I6

Assumption that Electrons come from Within the Cathode. ${ }^{16} 63$

Second Explanation of Ionization at Cathode......... 165

Cause which Determines the Amount of the Cathode Drop. I66

Ionization at Anode........................ I68

Discharge of Ions to Electrodes................ r. 70

Ionization of Gas Between Electrodes............... I7r

Velocity of the Ions..................... I76

Action of Arc not the Same as in an Electrolyte........ I 79

Variations in the Cathode Drop................. 180

Variations in the Anode Drop.................. $\mathrm{I}_{3}$

Variations in the Electric Force Through Arc......... 184

Effect Produced by Heating the Cathode............ 185 



\section{THE ELECTRIC ARC}

\section{CHAPTER I. INTRODUCTION.}

WE may well speak of our age as an Age of Artificial Light. Where formerly men were obliged to exist with only pine knots and candles to lighten their paths, we are now not only flooded with light but better and cheaper sources are being continually devised. The illuminating engineer counts that year lost which does not see some marked advance in the art of producing light. Among all the contestants for popular favor there is one source of light which stands as undisputed king. Other lights may answer the need of the worker at the desk, but when one wants light of the most brilliant sort, one turns to the electric arc.

Definition of Electric Arc. - An account of the electric arc might well begin with a statement concerning the time and place when man's eye was first dazzled by this phenomenon, but unfortunately we do not know when nor where this occurred. This is not because it took place so far back in the beginning of things, but because the earlier experimenters made no distinction between the arc and the spark.

They were, however, excusable in confusing these terms, for there is no logical and definite distinction 
between the momentary spark formed on opening a circuit and the continuous discharge which is called an arc. By gradually raising the E.M.F. and decreasing the resistance of a battery and shortening the distance between the terminals it is possible to pass continuously from a spark which lasts but a small fraction of a second to an arc which lasts indefinitely. Nor can we say that either the potential difference or the current of the momentary flash is necessarily either greater or less than that of the arc, and yet whatever might be the usage in a perfectly logical language, the actual usage is to make a distinction between these two phenomena. About all that can be said is that the discharge must last quite a while in order to be called an arc. "Quite a while" would appear to be sufficiently indefinite to meet the needs of this case.

It is much easier to distinguish between the arc and the continuous discharge in a vacuum tube where the current is small and the E.M.F. large, such as that shown in a Geissler or Crookes tube, which has also been called by some a spark. The current in this case is much smaller than with the arc, and the potential difference is very much higher. Moreover with the arc the potential difference between the cathode, as the negative terminal is called, and the layer of gas in its immediate neighborhood is small, while with the glow discharge it is large. With the former it may be as low as 5 or 6 volts, while with the latter it must be as high as 300 volts.

Difficulty is, however, experienced in making a distinction between the arc and the glow discharge in a vacuum when the latter has for the cathode a hot oxide of certain metals, as, for example, calcium oxide. In this case the drop in potential at the cathode may be as small as 
that with the arc and the current as large, ${ }^{1}$ and the only distinction between these two forms of discharge is that in the arc the cathode is heated by the current flowing through the gas, while with the hot calcium oxide the cathode must be heated by some external source.

Though it is not possible to make a perfect distinction between what is and what is not an arc, we shall not be far from the common usage, if we define an arc as consisting of a continuous current of several amperes or more, passing through a gas and having a cathode drop which is comparatively small. The most common form is that between carbons in air where the cathode drop is in the neighborhood of 9 volts and the current 5 amperes or more.

Appearance of Arc. - There are probably few people in civilized countries who have not seen an electric arc, and yet it would be nearly as truthful to say that there are few who have seen one. There are few who have not seen a frosted globe which was intensely luminous and was said to contain an electric arc, or who have not been blinded by looking too closely at an uncovered arc. On the other hand, there are few who have seen the difference between the luminous gas and the even more luminous terminals, who have seen the different shades of this gas as it varies from the violet of the brilliant center to the yellow of the faintly luminous edge, or have seen the fiery particles sent off from the white hot carbons, but without seeing these things one does not in reality see the electric arc.

As far as it is possible to reproduce such a view by means of an uncolored photograph, it is done in the accompanying illustration, which shows an arc between carbon terminals. Unfortunately this does not give

1 Phys. Rev., 29, 360; Ig09. 


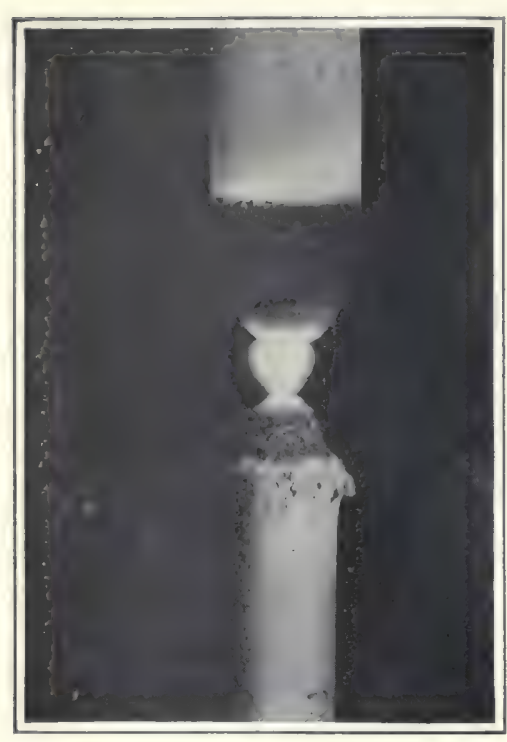

Fig. I.

distinctly the boundary between the carbon and the luminous vapor. An outline drawing as given in Fig. 2 may be helpful for this purpose. The carbon with the depression at the end is the positive carbon, or anode, as it is called, while the pointed carbon is the negative end, or cathode. The concave part of the anode, marked $c$, is intensely luminous and is called the crater. With this form of arc by far the greater part of the light comes from this crater. Since it is usually desirable to have the light thrown on the ground and not into the sky, the anode is placed above and the cathode below.

With homogeneous carbons this crater is apt to form at one side, with the result that much of the light is thrown to that side, while the region on the other side is left in comparative darkness. It was found that this could be largely remedied by making the carbons hollow and filling the hollow space with a softer quality of carbon. Such carbons

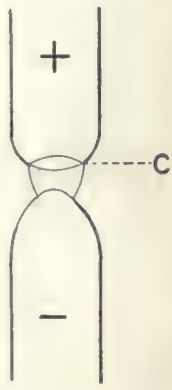

FIG. 2. are called cored carbons and are commonly used for the anode. Those which are not cored are called solid carbons. 
There is a point on the cathode which is also intensely luminous, but this is much smaller than the crater, so that much less light is received from it than from the anode.

The gas between the carbons is luminous and seen by itself would be considered brilliantly so, but compared with the crater it gives but little light. This at least is true of the form of the arc shown in the accompanying illustration. In the flaming arc, which is now coming into use, the vapor gives out more light than the crater. In that form the distance between the electrodes is longer and the vapor is more luminous than in the older form, and the crater less so.

The light from the vapor is not due to incandescent particles, but to the luminosity of the gas itself. This is shown by the fact that its spectrum is a line spectrum. The different parts of the vapor have different colors, which will be considered more in detail in connection with the spectrum of the arc.

Discovery of the Arc. - As has been stated, it is impossible to give the date of the first arc, because no attempt was made to distinguish between the arc and the spark. No mention was made by the first experimenters of the length of time occupied by the wonderful flash they described, and no one measured the current. The confusion between the two forms of discharge was the more excusable on their part, since they were trying to show that the electricity from chemical action was the same as that produced by friction and gave the same kind of flash, but it makes it inconvenient for one who tries to write a history of this work. ${ }^{1}$

1 The following is a list of a few of the articles appearing in the early part of last century: 
There is, however, no question but that it was an arc which Davy showed in his Bakerian lecture, in 1809 , when he described the discharge from rooo cells as a brilliant flame of from one-fourth inch to one-half inch in length. The name, "arc," appears to have been given by him in I $82 I^{1}$ because of the bow-shape of the arc when occurring between two horizontal electrodes.

Several experiments were made by the early workers on the transport of material in the arc. ${ }^{2}$ Some believed this phenomenon to be akin to that of electrolysis; some believed that what was seen in the arc was electricity itself, and though we must reject this view, we do it with the same feeling of sadness as that which we experience when we outgrow the fairy stories of our early years. Others thought that the light was given off by the hot particles which are driven off from the carbons.

The work of these experimenters is of little importance

Gilbert, Gilbert's Ann., 7, I6I; I80I.

Pfaff, Gilbert's Ann., 7, 248 and 516; 180r. 8, 340; I802.

Ritter, Gilbert's Ann., 9, 341; I80I.

Davy, Journal of the Roy. Inst., I, I66 and 209; I802.

Pepys, The Monthly Mag., 15, 259; 1803.

Cuthbertson, Nicholson's Journal, 8, 97; 1804.

Daniel, Phil. Trans., 92, 1839.

Grove, Phil. Mag., I6, 478; I840.

De la Rive, C. R., I2, 910; I84r.

Van Breda, C. R., 23, 262; 1846.

Despretz, C. R., 28, 757; I849. 29, 48 and 709; 1849 .

Matteucci, C. R., 30, 20I; 1850 .

Herwig, Pogg. Ann., I49, 521; 1873.

Violle, C. R., I17, 33; I893. I19, 949; I894.

Hertzfeld, Wied. Ann., 62, 439; 1897.

1 Phil. Trans., I8, I82I.

2 Hare, Sill. Journ., 3, 105; I821.

Silliman, Sill. Journ., 5, 108; 1822. 6, 342; I823. 10, 123; 1825. 
now, since we know that electricity can not be seen, that the phenomena of the arc are not the same as those of electrolysis and that the current is not carried by particles. That the arc is not an electrolytic phenomenon was shown most conclusively by Weedon ${ }^{1}$ and will be referred to at a later time. We may, therefore, leave the earlier work and give our attention to experiments of a somewhat later date.

1 Paper presented at the Electro-chemical Soc, at Washington, D. C., April, I904. 


\section{CHAPTER II.}

Arc in Air Between Carbon Electrodes.

The arc shown in Fig. I was one between carbon terminals. It is, however, possible to use any solid which is an electrical conductor or any metal in the liquid state for the electrodes. Electrolytes may even be used, if the E.M.F. is sufficiently high. Any other gas or a vacuum may take the place of air. However, only a few of the different forms of arc have been found to be of practical use, and of these the arc in air between carbon electrodes has been the most common. For the present we may give our attention to this form of arc.

The earliest carbon electrodes were pieces of charcoal which had been heated and plunged into mercury to make them better conductors, but in 1843 Foucault found that better results were secured by employing pencils cut from the hard graphitic carbon which is deposited on the interior of gas retorts. The carbon electrodes which are used to-day are usually made from retort or petroleum coke which has but a small percentage of ash. Lampblack has also been used for this purpose. Whichever is used, it is heated to a high temperature for several hours, to drive off any moisture or oil which may adhere to it. This heating increases the conductivity of the mass. It is then thoroughly mixed with some such binder as tar, and formed into rods of the desired size while under a pres- 
sure of 1500 to I 800 atmospheres. These rods are baked until all volatile substances are driven off and are then cut into the desired length.

If the rods thus formed are homogeneous they are called solid carbons. Cored carbons consist of an outer cylinder which is made by the same process as that just described and a core which is filled in after the outer cylinder is finished. According to Mahlke ${ }^{1}$ the core consists of two parts of baked lampblack and one part of potassium silicate. The core usually has a diameter about one-fifth that of the whole carbon.

\section{Relation Between Potential Difference, Current and} Length of Arc. - One of the first questions for us to consider is that concerning the amount of E.M.F. necessary in order to maintain the arc. The first accurate measurements taken for the purpose of answering this question were made by Edlund in $1867 .^{2}$ He found that $r=m+n l$, where $r$ is the "apparent resistance," that is the ratio between the voltage at the terminals of the arc and the current; $l$ its length; and $m$ and $n$ quantities which are constants as long as the current is constant. He further stated that the voltage of the arc is independent of the current, and that the "true" resistance, represented by the term $n l$, is proportional to the length and increases as the current decreases.

There were at that time no definite units for measuring electrical quantities and no dynamo for producing the current, so that the accuracy of Edlund's results seems to have been the result of good fortune as well as of careful

1 Elec. World., 57, 672; I9II.

2 Pogg. Ann., 131, 586; 1867. 133, 353; 1868. 134, 250 and 337; 1868. 139, 354; 1870. Wied. Ann., 26, 518; 1885 . 
work. In his explanations he was not so fortunate. His explanation was that this "apparent resistance" was partly due to a counter E.M.F., which was equal to the current times the $m$ in his formula, and partly to a "true" resistance corresponding to the term $n l$. The counter E.M.F. he found equivalent to 23 Bunsen cells, which is approximately 40 volts.

Though this explanation was not fortunate in the sense of being correct, it was very fortunate in that it caused much discussion on the part of other scientists and led to many new experiments. These have dealt on the one hand with the accuracy of his formula, and on the other with the truth of his explanation. It is scarcely of value to consider in detail the data given by the different experimenters. If we should do so, we would find that they differ greatly among themselves, and the real significance of their work is that there are rarely two carbons which give the same results. Even when two carbons are exactly alike the data observed will not be identical unless the conditions are the same. ${ }^{1}$

For example, it has been shown by Mrs. Ayrton ${ }^{2}$ that the voltage of an arc when first started is quite different from what it is after it has reached a steady condition. The time required to reach this state depends largely on

1 Ayrton and Perry, Proc. Phys. Soc., 5, 197; 1882.

Cross and Shepard, Proc. Amer. Acad. Sc., 22, 227; 1886.

Frölich, Elektrot. ZS., 4, 150; 1883 .

Nebel, Centralbl. f. Elektrot., 8, 517 and 619; 1886.

Uppenborn, Centralbl. f. Elektrot., 9, 633; I888.

Luggin, Centralbl.f. Elektrot., 10, 567; 1888.

Granqvist, Beib., 22, 243; 1898.

Thompson, Elec. Rev., 27, 262; 1895.

2 Mrs. Ayrton's "Electric Arc," p. 107. 
the shape which the ends of the carbons have when the arc is started, but even when their shape at the beginning is that which they finally take, it is many minutes before the arc reaches a constant condition.

The variation in voltage during an hour's run is shown in Fig. 3. The current during this time was kept at Io

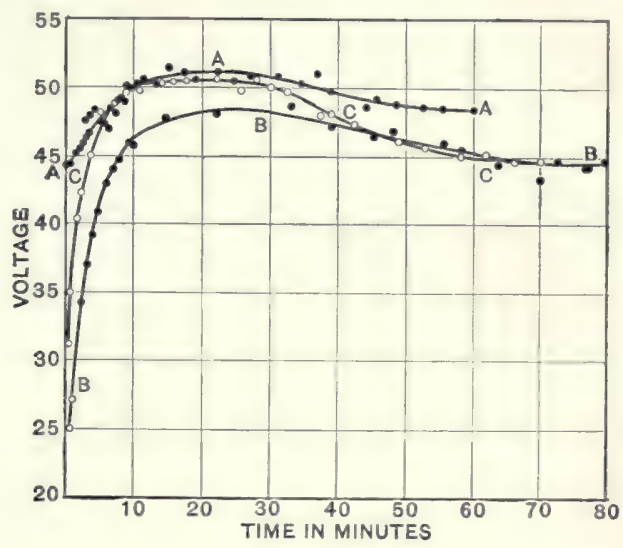

Fig. 3.

amperes, and the length of the arc $3 \mathrm{~mm}$. The negative carbon in each case was a solid carbon $\mathrm{I}_{5} \mathrm{~mm}$. in diameter. This had been shaped by being previously used for a long time with an arc which was also $3 \mathrm{~mm}$. long having a current of ro amperes. In curve $A$ the positive carbon was solid with the end filed flat before the arc was started. In $B$ the positive carbon was cored with the end filed flat. In $C$ the positive carbon was cored with a crater mechanically formed before the arc was started. With these carbons the arc had hardly reached a constant condition at the end of an hour. 
Even when the arc has reached a constant condition with a given current it requires several minutes for it to become steady again if the current is changed. This is shown in the curves given in Fig. 4. The positive carbon was I $3 \mathrm{~mm}$. in diameter and was cored, the negative I I $\mathrm{mm}$. and solid. The length of the arc in the upper curve was $4 \mathrm{~mm}$. and in the lower $3 \mathrm{~mm}$.

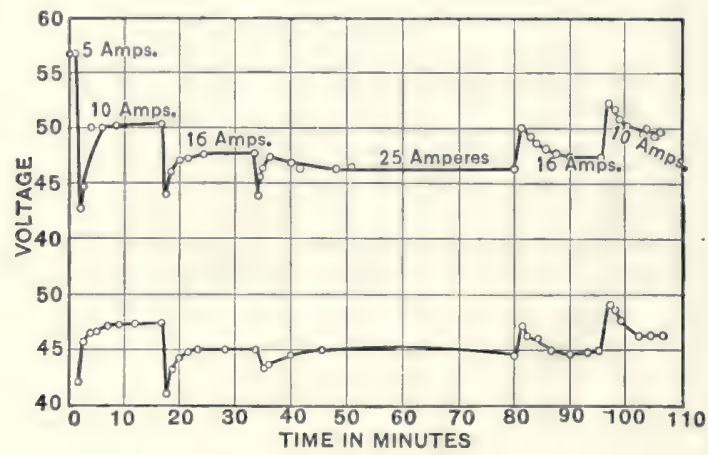

FIG. 4.

The most careful examination of the relation between current and voltage has been made by Mrs. Ayrton, and some of her results are shown in the accompanying figures. Fig. 5 shows a series of curves, called " characteristic" curves, giving this relation for different lengths of arcs with solid carbons. They show clearly the difference between the resistance of the arc and that of a metal. With a metal the potential difference increases as the current increases and is directly proportional to it. Here the voltage decreases when the current increases. It seems at first as if the greater the cause the less the effect, but it should be noticed that while the voltage decreases the 
amount of energy used in the arc increases. The greater amount of heat thus produced causes the conductivity of the gas between the electrodes to be greater, and the conductivity increases so rapidly that the voltage needed actually becomes less with the larger currents. Further

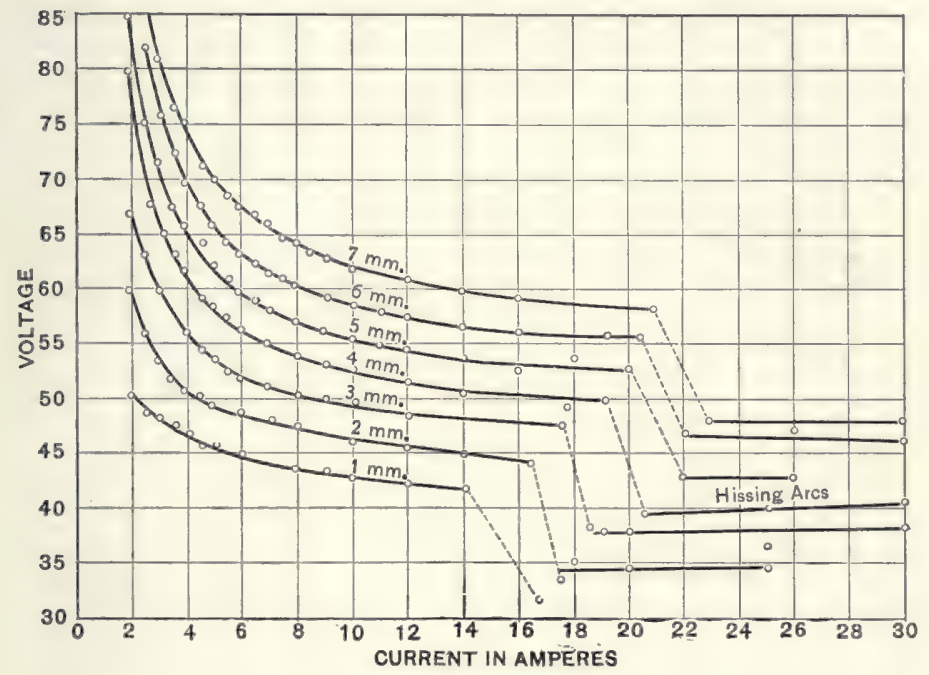

FIG. 5 .

consideration of this will be given in the chapter on the theory of the arc.

After decreasing to nearly a constant value the potential suddenly drops, as is indicated in the dotted lines in the curve. At this point the arc begins to emit a loud, hissing sound. Such an arc is called a hissing arc. This will be more fully described in a following paragraph.

A similar set of curves is shown in Fig. 6, where the positive carbon is cored and the negative one is solid. 
Mrs. Ayrton found that with the solid carbons which she used the relation between the potential difference, current and length of arc could be expressed by the equation

$$
E=38.88+2.07 l+\frac{11.66+10.54 l}{I},
$$

where $E$ is the potential difference between the terminals in volts, $I$ the current in amperes, and $l$ the length of the

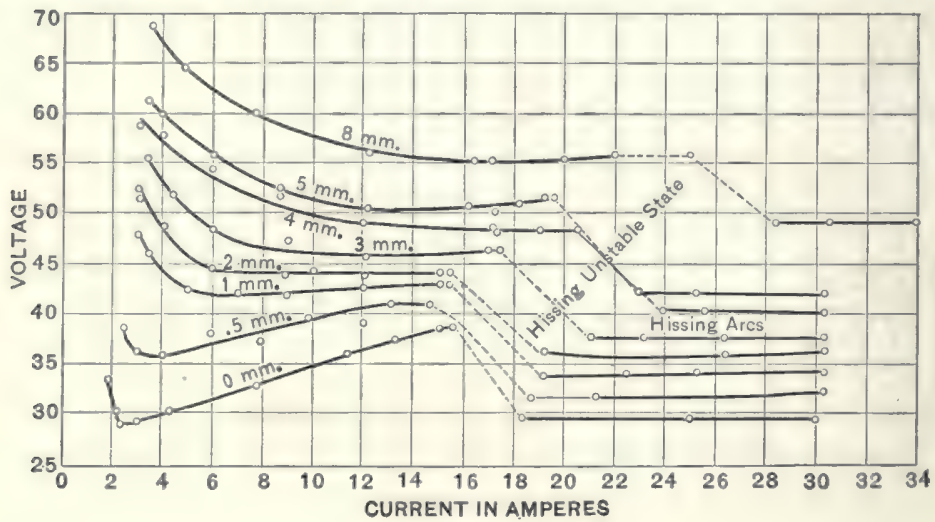

FIG. 6.

arc in millimeters. For cored carbons the relation could not be expressed by a simple equation. She believed that this was due to the change which occurs when the crater of the anode begins to cover more than the core of the carbon.

The power used in the arc is, of course, equal to the potential difference times the current, so that the equation for the power used in the arc with solid carbons according to Mrs. Ayrton's data is

$$
P=(38.88+2.07 l) I+\mathrm{II} .66+\mathrm{I0} .54 l,
$$


where $P$ is the power used. When this equation is plotted, using power for ordinates and current for abscissæ, we have a straight line.

When an arc is very long, the equations given by Mrs. Ayrton do not hold, as has been shown by Duddell. ${ }^{1}$ The curve given by her equation and that found experimentally by Duddell are plotted in Fig. 7. Duddell also found a similar difficulty when the currents were less than I.5 amperes, the voltage then being less than that calculated from Mrs. Ayrton's equations.

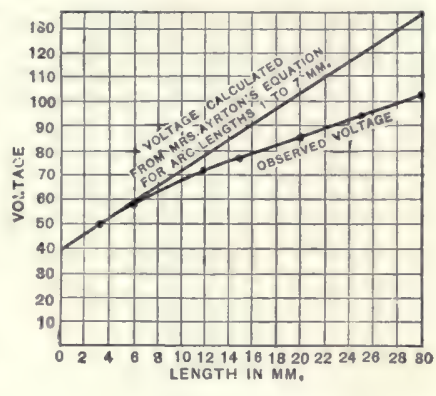

FrG. 7 .

The characteristic curves for arcs between solid carbons in air and with currents as small as 0.6 ampere were observed by Malcolm and Simon. ${ }^{2}$ They give the equation $E I=49+60 I$, when the length is $8 \mathrm{~mm}$., and $E I=3 \mathrm{I} .5+{ }_{5} \mathrm{I} .5 I$, when it is $4 \mathrm{~mm}$.

We have here been giving the relation between the potential difference and current for arcs that have been running sufficiently long to have reached a steady condition. This is what one usually wishes to know. An engineer, for example, wishes to know the power needed by a lamp under certain constant conditions. But there are also times when one wishes to know something about the relation between potential difference and current when they are changing rapidly. Such knowledge, for example, is desirable when using arcs in wireless telephony. Curves

\footnotetext{
1 Phil. Trans., 203, A, 338; 1904.

2 Phys. ZS., 8, 47I; I907.
} 
giving the relation for steady conditions have been called by Simon " "statical" characteristic curves; those giving the relation with changing conditions were called "dynamical" characteristic curves.

The dynamical curves are found by comparing the potential differences with the currents for different phases of an alternating-current arc, or when an alternating current is superimposed on a direct current which is maintaining an arc. The discussion of such curves will, therefore, be considered in connection with alternating-current arcs.

E.M.F. Required as Distinct from Voltage of Arc. - Arc lights are commonly maintained by constant current dynamos. Under such conditions there is no need of putting resistance in series with the arc in order to insure its stability. But occasionally an arc is connected to some source maintaining a constant E.M.F., as, for example, a constant potential dynamo. When this is done, it is necessary to put resistance in series with the arc, as was pointed out by Blondel. ${ }^{2}$ The curves which have been given show the potential difference between the electrodes for different currents, but they do not show the required E.M.F. of a source having a constant voltage. Such E.M.F. can be found as follows:

Let us assume that the potential difference is $E$ when the current is $I$ for any given length $l$. If now the dynamo gave exactly $E$ volts, and there was no resistance in the circuit, the system would be in a condition of unstable equilibrium. For if there were any fluctuations either in the dynamo or in the arc which gave a momentary

1 Phys. SZ., 6, 302; 1905.

${ }^{2}$ Lum. Elec., 42, 62I; I89I. 
increase in the current, the potential difference needed for the arc would be less than the E.M.F. of the dynamo, and under such conditions the current would increase still further. This would cause a further decrease in the voltage used by the arc and if the E.M.F. were maintained, this process would continue until the dynamo was burnt out.

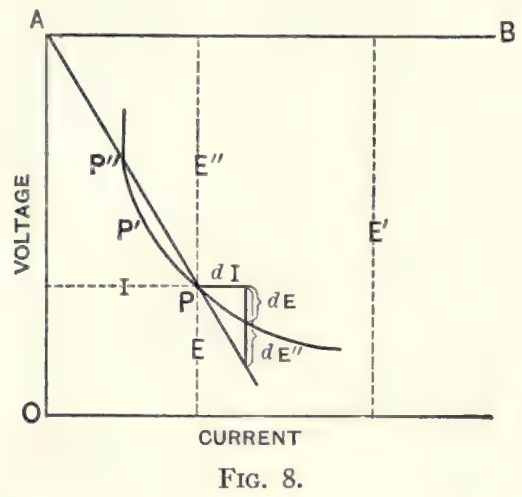

If the current momentarily decreased, a higher potential difference would be needed than the dynamo was giving, and the arc would be extinguished.

On the other hand if there is to be resistance in the circuit, then the E.M.F. of the dynamo must be greater than the voltage of the arc. In order to determine how much greater the E.M.F. must be, we need to remember that there must be enough resistance in series with the arc, so that any increase in the current will produce a greater increase in the drop in potential through the resistance than the decrease in voltage occurring in the arc. This can perhaps be made clearer by referring to Fig. 8. Let the curve $P P^{\prime} P^{\prime \prime}$ represent the relation between the cur- 
rent flowing through the arc and the potential difference at its terminal. The ordinates represent the voltage and the abscissæ the current. $E^{\prime}$ is the E.M.F of the dynamo. This is partly used in maintaining the potential difference at the terminals of the arc and partly in maintaining the drop through the resistance. This latter we will call $E^{\prime \prime}$. As long as this resistance is constant, $E^{\prime \prime}$ will be directly proportional to $I$, the current flowing through the arc. The vertical distance from the line $A B$ to any point on the straight line $A P$ represents the drop through this resistance, while the abscissa of the point represents the corresponding current.

It is conceivable that with any given resistance there should be two different currents corresponding to the points $P^{\prime \prime}$ and $P$, where the straight line cuts the curve $P P^{\prime} P^{\prime \prime}$. For at both of these points the sum of the drop in potential through the arc and that through the resistance equals the E.M.F. of the dynamo. With other currents their sum would either be too small or too great.

With the current corresponding to the point $P$ there is stable equilibrium. For if the current should be momentarily increased, $E$ will decrease by an amount $d E$, but at the same time $E^{\prime \prime}$ will increase by an amount $d E^{\prime \prime}$ and this is greater than $d E$, so that the two together would require an increase in the E.M.F. equal to $d E^{\prime \prime}-d E$. In other words this increase in current could not be maintained, if the E.M.F. of the dynamo remained constant.

Similar reasoning will show that any momentary decrease in the current could not be maintained without a decrease in the E.M.F. of the dynamo, so that $P$ represents a point of stable equilibrium.

In a similar way it can be shown that the point $P^{\prime \prime}$ 
represents a point of unstable equilibrium. If all the conditions were absolutely constant, a small current represented by the abscissa of $P^{\prime \prime}$ could exist, but any increase in the current would cause $E$ to decrease faster than $E^{\prime \prime}$ would increase, so that the sum of the two would be less than the E.M.F. supplied by the dynamo. This would cause a further increase in the current and it would continue to increase until it became equal to $I$, the amount corresponding to the point $P$.

In a similar way it can be shown that any decrease from the amount corresponding to the point $P^{\prime \prime}$ would cause the arc to be extinguished, so that $P^{\prime \prime}$ represents a point of unstable equilibrium.

As the resistance in series with the arc is varied the slope of the line $A P$ will change, but there will be a point where there is stable equilibrium as long as this line intersects the curve $P P^{\prime} P^{\prime \prime}$. The limiting case will occur when $A P$ is tangent to $P P^{\prime} P^{\prime \prime}$.

To state mathematically what has been given above, the condition for stable equilibrium is that

$$
-\frac{d E}{d I}<\frac{d E^{\prime \prime}}{d I}, \quad \text { but } \frac{d E^{\prime \prime}}{d I}=R ; \quad \therefore-\frac{d E}{d I}<R,
$$

where $R$ is the resistance in series with the arc.

For many of the arcs it is approximately correct to say that $E=a+\frac{b}{I}$, where $a$ and $b$ are constants for any given length. In such cases it would follow that

$$
-\frac{d E}{d I}=\frac{b}{I^{2}} ; \quad \therefore \frac{b}{I^{2}}<R \text {, or } \frac{b}{I}<R I .
$$

But $\quad E^{\prime}=E+R I ; \quad \therefore E^{\prime}>E+\frac{b}{I}$.

That is, the E.M.F. of the dynamo must be greater 
than the potential difference between the terminals of the arc plus the amount indicated by $b / I$. For example, if we take the values of $a$ and $b$ for the arc $5 \mathrm{~mm}$. in length, as given by Mrs. Ayrton, we have $E=50+64 / I$. Therefore, for to amperes the resistance in series with the arc must be more than $0.64 \mathrm{ohm}$ and the E.M.F. of the dynamo must be more than 62.8 volts, although the potential difference between the terminals of the arc is only 56.4 volts. For 5 amperes the resistance must be more than $2.48 \mathrm{ohms}$ and the E.M.F. more than 75.6 volts. This is quite in agreement with the well-known fact that the smaller the current the greater the resistance that must be in series with it.

The energy used in the resistance is $E^{\prime \prime} I$. If we again assume that $E=a+b / I$, we have $E^{\prime}>E+b / I$. From this and the equation $E^{\prime \prime}=E^{\prime}-E$ it can be shown that $E^{\prime \prime} I$ must be greater than $b$. The energy used in the arc is $a I+b$, so that the ratio of that lost in the resistance to that used in the arc is greater than $\frac{b}{a I+b}$. This becomes smaller as $I$ becomes larger. That is, the percentage of energy necessarily wasted in the resistance, when a constant voltage dynamo is used, is less the greater the current. This, of course, does not state what is the best current to use in practice, for there is still the question as to the relation between the light efficiency of the arc and the current, a question that will be considered in the chapter on photometry.

It should be noted that to have stable equilibrium it is necessary that the stability be not destroyed either because of the sudden change in potential difference between the electrodes when the current changes, or by the slower 
change considered above which continues to occur for several seconds. However, since the sudden change is commonly less than the slower one the arc will be in equilibrium for the sudden change, if it is for the slower one.

Hissing Arc. - With the currents which are ordinarily used the arc makes little or no noise. Such an arc is known as a "silent arc." With larger currents the arc gives a hissing sound which becomes very loud and disagreeable under certain conditions. Such an arc is known as a "hissing arc." The voltage required is here much smaller, as is shown in the lower parts of the curves in Figs. 5 and 6.

The correct explanation of this appears to have been given by Mrs. Ayrton. ${ }^{1}$ She found that when the crater fills somewhat symmetrically the end of the anode, the arc is silent; when it extends up the side, the arc hisses. Moreover when the arc is enclosed so that no oxygen can get to it, or is placed in nitrogen, there is no hissing, while there is hissing when it is in oxygen. She believed that the hissing is due to oxygen coming in contact with the crater, and combining with the hot carbon. This chemical action is supposed to cause a sudden decrease in the voltage and a corresponding increase in current. The increase in current drives the air away from the crater, and this again causes a higher voltage with decrease in current. Thus, the arc fluctuates from one condition to the other with the accompanying production of sound waves. ${ }^{2}$ That oscillations in

1 Journ. Inst. Elec. Eng., 28, 400; r899. Mrs. Ayrton's “Electric Arc," p. 299.

2 Further experiments were performed by the following:

Niaudet, C. R., 92, 7II; I88I.

Gime, Lum. Elec., 18, 556; 1885.

Craveth, Elec. World, I9, I95, and 20, 227; 1892. 
the current accompany the hissing, has been shown by Firth and Rogers, ${ }^{1}$ and by Duddell and Marchant. ${ }^{2}$

Fall of Potential in Different Parts of the Arc. - The relation between the potential difference and the current through the arc is so different from that given by Ohm's law that it is a matter of interest to examine the potential in different parts of the arc and to find whether all parts show the same peculiarity. To do this some point in the arc must be connected to a measuring instrument by a conducting substance, such as a metallic wire. But any wire placed in the arc is immediately vaporized. Even a carbon filament will not answer, since it burns away rapidly. The only conducting substance which can be used is a carbon pencil. But a carbon pencil distorts the form of the arc and changes the conditions which existed before its introduction. ${ }^{3}$

However, approximate results can be found by this method, and these always show a considerable difference in potential between the anode and a pencil placed as close to it as possible. This is called the anode drop. There is a similar difference in potential in the neighborhood of the cathode, which is called the cathode drop. Between these there is a gradual change in potential as in a wire. This is illustrated by Fig. 9 taken from some of Mrs. Ayrton's data, which will be given in the following paragraph.

Measurements of these quantities have usually been made with either a voltmeter or a galvanometer. This involves a passage of electricity from the gas to the exploring electrode. As is well known, there is usually a large

1 Phil. Mag., (5), 42, 407; 1896.

2 Journ. Inst. Elec. Eng., 28, 78; I899.

3rs. Ayrton's " Electric Arc," p. 210. 
drop in potential at the surface between a gas and a solid when a current passes from one to the other. Such a drop here might introduce a large error. But this objection disappears when an electrometer is used instead of a voltmeter and it has been shown ${ }^{1}$ that the two instruments give the same readings when used to measure the potential in the arc. It, therefore, seems safe to assume that in this

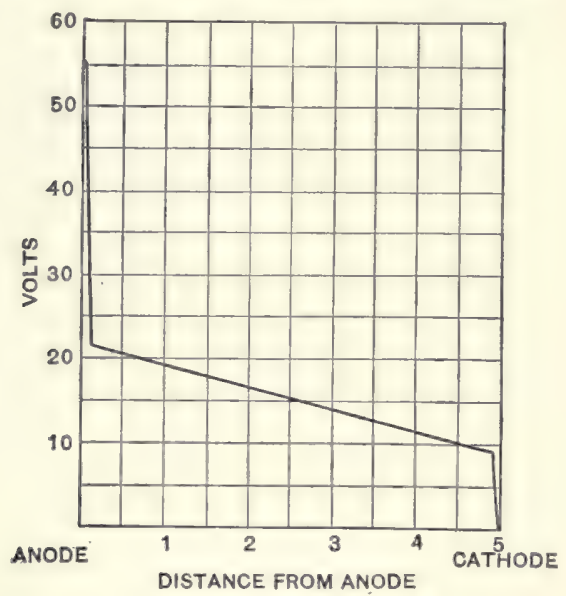

Fig. 9.

case the exploring carbon becomes so hot and the vapor of the arc is such a good conductor that there is no appreciable drop in potential between the pencil and the gas about it.

This fact is not in agreement with an experiment of Fleming's. ${ }^{2}$ He was not able to find any current flowing through a circuit from an exploring pencil to the cathode. If this were correct, it would not be possible to measure

1 Phys. Rev., 19, I19; I904.

2 Proc. Roy. Soc., 47, I23; I8go. 
the cathode drop with a voltmeter or galvanometer, a measurement which has been made many times. Probably in Fleming's experiment the pencil was not inserted far enough into the arc to become thoroughly hot. If the pencil was comparatively cold, it would be quite possible to make the observations as they were made by him.

Measurements of the anode and cathode drops have been made by Lecher, ${ }^{1}$ Uppenborn, ${ }^{2}$ Luggin,${ }^{3}$ and Fleming. ${ }^{4}$ But the most accurate work is that given by Mrs. Ayrton, who gives

$$
E_{a}=31.28+\frac{9+3 . \mathrm{I} l}{I}
$$

as the equation for the anode drop when both carbons are solid, where $E_{a}$ is the anode drop, $I$ the current, and $l$ the length of the arc in millimeters. She also gives

$$
E_{c}=7.6+\frac{\mathrm{I} 3.6}{I},
$$

where $E_{c}$ is the cathode drop. When cored carbons are used she found that the potential difference is in general two or three volts lower than when both carbons are solid, and that the cathode drop is about I volt lower.

The direct measurement of the fall of potential through the gaseous part of the arc cannot be satisfactorily accomplished. It requires the introduction into the arc of two carbon pencils, and this distorts the arc so much as to make nearly valueless any measurements which may be

1 Centralbl. f. Elektrot., I0, 48; I888. Wied. Ann., 33, 609; 1888.

2 Centralbl. f. Elektrot., 10, I02; I888.

3 Centralbl. f. Elektrot., 10, 567; 1888.

"Proc. Roy. Soc., 27, I18; 1894.

"Mrs. Ayrton's "Electric Arc," p. 222. 
made. A few measurements were made by myself ${ }^{1}$ in this manner, and the electric force was found to be 2.34 volts per millimeter for a current of ro amperes, but no great. accuracy can be claimed for these measurements.

It has generally been assumed that the electric force could be found by observing the increase in voltage when the length of the arc was increased and that it is equal to the ratio of the increase in voltage to the increase of length, the current remaining constant. This would be correct, if changing the length of the arc did not change either the anode or cathode drop, but Mrs. Ayrton found that such a change did exist in the anode drop. However, we can arrive at a fair approximation by following this method and making a correction for this change. If, therefore, we assume the equation for $E$, given on page I4, and that for the anode drop given on page 24, we find the value for the electric force to be $2.07+7 \cdot 44 / I$. This will give a value of $2.8 \mathrm{I}$ volts per millimeter when the current is Io amperes, which is not far from that found experimentally by myself.

We thus see that the peculiar relation which exists in the arc between voltage and current must be ascribed to all of the three parts. The voltage of each part decreases when the current increases. The decrease in the anode drop is greater than that in the cathode drop, while the decrease in the drop of potential through the gaseous part of the arc depends, of course, on the length of the arc. However, with arcs more than $6 \mathrm{~mm}$. long more than onehalf of the total decrease occurs in the gaseous part.

Counter E.M.F. of the Arc. - As has been stated Edlund found that the "apparent resistance" of the arc equals 1 Phys. Rev., I9, I22; I904. 
$m+n l$, where $m$ and $n$ are constants and $l$ is the length of the arc. He believed that the first term of this expression was due to a counter E.M.F. Others did not agree with him, and the discussion then started has continued to the present time. This discussion has been carried on partly because there have been differences of opinion as to facts, but more often because there has been no agreement as to the meaning of the terms used. In the beginning there was too little known to admit of any clear definition of the terms, "counter E.M.F." and "resistance," and though there have since been clear definitions of these terms, there has been no general agreement as to their use, and often no good understanding of how others were using the terms.

It would appear that the term "counter E.M.F." should mean the same when applied to the arc as when applied to a motor or a storage cell. In those cases we find: first, that the potential difference between the terminals of a cell or motor does not obey Ohm's law; secondly, that there is an E.M.F. remaining after the impressed E.M.F. has been removed and that this is of sufficient magnitude to account for the deviation from Ohm's law; and thirdly, that in the majority of cases the electrical energy is changed into something besides heat energy.

In the arc only the first of these properties is found to exist. In fact the arc deviates from Ohm's law much as other forms of discharge through gases deviate, where the expression "counter E.M.F." has never, I believe, been applied.

In the beginning of the investigation on the arc it was not known that this was the only point of similarity between the arc and a cell, and writers using the word no doubt had in mind a counter E.M.F. identical with that of 
the cell. Later writers have used the term merely to mean that the potential difference of the arc deviates from Ohm's law in a definite manner, and have so defined it. Yet no one seems to have pointed out that the meaning of the term as defined by Duddell, for example, is not the same as the meaning which Edlund had in mind, nor the meaning generally given to the term in physics. This has led to more or less confusion in what has been written on the subject during the past few years. For example, one occasionally sees the statement that it has at last been definitely shown that there is a counter E.M.F. in the arc. The fact is that on the one hand it has neither recently nor at any other time been shown that the arc has more than the first of the three peculiarities mentioned above, and on the other it has never been doubted but that there was this one point of similarity between the arc and the cell.

The methods which have been used to test the existence of a counter E.M.F. may be divided into two general classes: First, methods which attempt to find a counter E.M.F. by measuring the resistance of the arc; and secondly, those attempting to find it by measuring the E.M.F. remaining between the carbons after the impressed E.M.F. has been removed, or what we may call the residual E.M.F. of the arc.

Those who attacked the problem by measuring the resistance of the arc have assumed that the counter E.M.F. was the difference between the potential difference at the terminals of the arc and that part of it which appeared to obey Ohm's law. In other words that $E^{\prime}=E-R I$, where $E^{\prime}$ is the counter E.M.F., $E$ the potential difference at the terminals, $R$ the resistance of the arc, and $I$ the current. 
Meaning of "Resistance of the Arc." - We find, however, that "resistance of the arc" has meant four different things with different experimenters, and with these different meanings there are corresponding differences in what is meant by counter E.M.F.

First of all it has been used to mean the ratio between the potential difference at the terminals and the current. This, however, has usually been called the "apparent resistance."

Secondly, it has been used to mean the term $n l$ in the expression $r=m+n l$, which was used by Edlund, namely, the part of the apparent resistance which was proportional to the length of the arc. This Edlund called the "true resistance."

At a later time it was used to mean the ratio between a small increase in $E$ and the corresponding increase in $I$, or what we may call $d E / d I$. An approximation to this was made by those who used some modification of the Wheatstone bridge.

Finally, it was pointed out by Luggin ${ }^{1}$ that any change in the current through the arc produced a corresponding change in both the vapor of the arc and the shape of the carbon, so that one does not have the same arc after the current has been increased as before. The methods which are modifications of the Wheatstone bridge do not correctly measure either the resistance of the arc in its first condition, nor its resistance after the current has been increased. This difficulty has been clearly stated by Mrs. Ayrton, ${ }^{2}$ Duddell ${ }^{3}$ and others. They have, therefore, de-

1 Centralbl. f. Elektrot., 10, 567; 1888.

2 Mrs. Ayrton's "Electric Arc," p. 400.

s Phil. Trans., 203, A, 306, 1904. 
fined the true resistance of the arc as the ratio between a small change in potential difference and the corresponding change in the current, when the change is made so small, or for so short a time, that the condition of the arc itself does not vary. In other words the resistance as thus defined is the partial derivative of the potential difference with respect to the current which we shall indicate by $\partial E / \partial I$.

This would undoubtedly be the correct way of defining the resistance, if it were not for the doubt which must exist in one's mind as to whether it is possible to have a change in current without having a change in the condition of the arc. If the current in the arc is carried by ions, as we have reason to believe, it is not possible to change the current without changing either the number of the ions or their velocity. In either case there would be a change in the condition of the gas. However, this definition may properly be considered as giving a limiting condition toward which a close approximation may be made, although it would be better to avoid the use of the word "resistance" entirely when speaking of the arc. It is commonly avoided when speaking of the discharge of electricity through vacuum tubes, and there is the same reason for avoiding it here.

If we pass to other methods of attacking the problem we find that Swendler ${ }^{1}$ gave the value of the counter E.M.F. as 2 volts, but since he did not divulge his method of arriving at this conclusion, we are unable to judge of the importance of his work.

As far as we know the first experimenter to use a method different from that of Edlund's was von Lang ${ }^{2}$ who en-

1 Lond. Elec., 2, 107 and I17; 1879.

2 Centralbl. f. Elektrot., 7, 443; I885. Wied. Ann., 26, г45; 1885. 
deavored to measure the resistance of the arc by a modification of the Wheatstone bridge, shown in Fig. Io. Two $\operatorname{arcs} L_{1}$ and $L_{2}$ are operated by a battery $B_{1} B_{2}$. This circuit is connected to the Wheatstone bridge at $B$ and $A$. If all the resistances are known except those in the arcs, their resistances may be computed. It is evident that there is great difficulty in balancing a bridge with this arrangement. It is in fact surprising that von Lang was

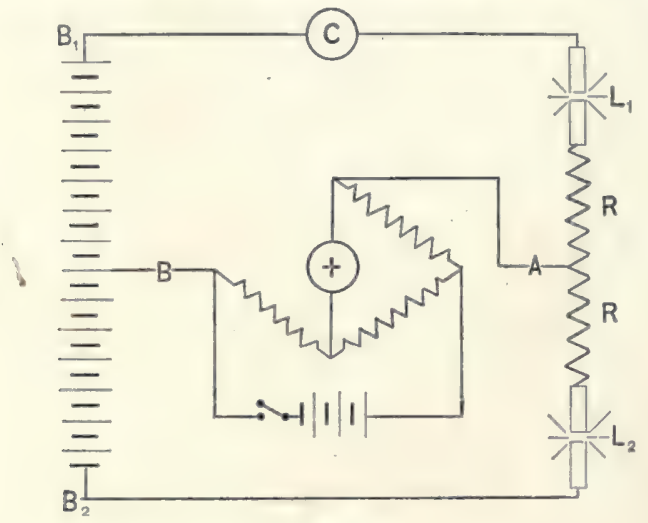

FIG, IO.

able to give any definite value whatever to the resistance as thus found. He found the resistance to be $\mathrm{I} .82 \mathrm{ohms}$ and concluded that the counter E.M.F. was 38.9 volts. This method gave approximately the value of the resistance as defined the third way, namely, $d E / d I$.

Attempts were then made to find the resistance when the change in the current was made much smaller, that is, ${ }^{1}$ to get the value indicated by the expression $\partial E / \partial I$. Arons attempted to do this by using a different modification of the Wheatstone bridge. The arc with the battery produc-

1 Wied. Ann., 30, 95; I887. 
ing it was used as one branch of the Wheatstone bridge. Instead of the battery ordinarily used with the bridge a transformer was used and instead of a galvanometer a dynamometer. The three arms of the bridge were adjusted until no current was shown by the dynamometer. The resistance of the arc could then be determined. The resistance was found to be approximately $2 \mathrm{ohms}$ and the counter E.M.F. 40 volts. Luggin ${ }^{1}$ used a different modification of the bridge and Firth ${ }^{2}$ still another, both getting results similar to those of Arons.

"Negative Resistance." - In I895 Ayrton ${ }^{3}$ concluded from the result of a few simple experiments that the resistance of the arc was negative. What he measured was $d E / d I$. It had been previously pointed out by Luggin that this quantity was negative, and it has since been shown even more clearly by the curves given by Mrs. Ayrton. ${ }^{4}$ If one is to define resistance as meaning $d E / d I$, there is no question but that the resistance of the arc is a negative quantity, but of all the uses of the word this is perhaps the least justifiable, and to speak of a negative resistance is, to say the least, misleading.

The word, resistance, means primarily something which hinders the movement of some object. An electrical resistance means something which hinders the flow of an electric current, and the most natural meaning of the expression "negative resistance" would be something which helps the flow of the current. It is needless to say that the resistance of the arc does not help the flow of current.

${ }^{1}$ Centralbl. f. Elektrot., 10, 567; 1888.

2 Mem. and Proc. Manchester Lit. and Phil. Soc., (4), 9, I39; 1895.

3 Mrs. Ayrton's "Electric Arc," p. 75.

4 Mrs. Ayrton's "Electric Arc," p. Ir3. 
Firth and Rogers ${ }^{1}$ superimposed an alternating current on the direct current through the arc, and the resistance of the arc to this alternating current was taken as its resistance. Frequencies between 7 and 250 complete alternations per second gave the same resistance. They, therefore, believed that the changes in the current were so rapid that there were no corresponding changes in the arc. The value of the resistance which they found varied much with different carbons and with different currents. Values ranging from 2 to $-2 \mathrm{ohms}$ are given by them. The corresponding values of the counter E.M.F. were in the neighborhood of 40 volts.

Following the investigation of Firth and Rogers was a careful examination of the counter E.M.F. and resistance by Duddell. ${ }^{2}$ His principal advance over the work of others consisted first in devising a method by which one could test whether the arc remained constant during the change in current or not, and secondly, in using much higher frequencies than those which had previously been used.

To decide whether the arc changed any or not he imposed a small alternating current on the direct current which was passing through the arc, and determined whether this alternating current was in phase with its E.M.F. or not. Ordinarily, when an alternating current is thus used, it lags behind its E.M.F. Duddell assumed that if the two were in phase the condition of the arc had not changed during the change in current. He found that for low frequencies with solid carbons the oscillations were r8o degrees out of phase, and that to have the two

1 Phil. Mag., (5), 42, 407; 1896.

2 Phil. Trans., 203, A, 305; I904. 
in phase with such carbons it was necessary to have frequencies of 100,000 or more per second. For cored carbons 26,000 alternations per second were needed.

When the alternating current was in phase with its E.M.F. the ratio between the E.M.F. and the current was taken as the "true resistance" of the arc, and the counter E.M.F. was found from the formula $E^{\prime}=E-R I$. The value of $E^{\prime}$ as found by him varied from II.2 to I8.5 volts with different currents and different carbons.

"Forward E.M.F." - He also endeavored to find the part of the arc where this counter E.M.F was situated. For this purpose he inserted an exploring pencil in the arc and found the potential difference between this and the anode, both for the direct and for the superimposed alternating current. From this data he computed the counter E.M.F. near the anode, using the same method as that given above. This he found to be approximately I7 volts. A similar set of readings gave a "forward E.M.F." near the cathode of 6 volts, the two accounting for the II volts which were found in this particular case for the whole arc.

This again is a use of words which in one sense is entirely correct, and yet is entirely misleading. Duddell undoubtedly found the value of $E-R I$ near the cathode to be a negative quantity, and since he had called this a " counter E.M.F." when it was positive, it is not surprising that it should be called a "forward E.M.F." when it was negative. Yet to do so gives one the impression that there is an E.M.F. at the cathode of the order of 6 volts, which changes some other form of energy into electrical energy. In reality there is no reason whatever to suppose that there is any such E.M.F. at that point. All that Duddell's 
experiment showed was that $\partial E / \partial I$ was greater than $E / I$ between the gas of the arc and the cathode. In other

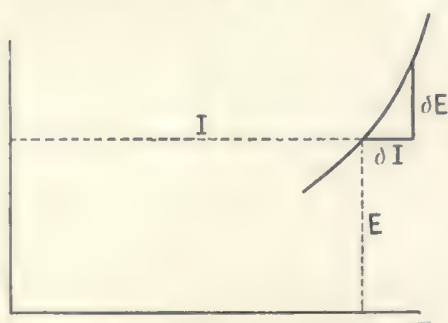

Fig. II. words, that the form of the curve showing the relation between the cathode drop and the current was that shown in Fig. Ir. This is the same kind of a curve that one finds when examining the relation between the potential difference and the current for discharge produced by Röntgen rays. No one ever speaks of a "forward E.M.F." with such discharge and there would appear to be no more reason for using that expression in connection with the arc.

Pollock ${ }^{1}$ explained this "forward E.M.F." of Duddell's as being due to the tendency of a hot cathode to send negative ions out from it. This tendency undoubtedly exists and in this case it would assist the flow of current, but if it assisted enough to produce a forward E.M.F., there would be a rise in potential at the surface of the cathode instead of a drop, and a change of heat energy into electrical energy, while in reality we find there a change in the opposite direction. It is altogether possible that this change of energy would be greater, if the emission of negative ions did not exist, but we have no evidence that the difference between what the cathode drop would be and what it is, is the same in amount as the "forward E.M.F." which was found by Duddell.

Heinke $^{2}$ computed the counter E.M.F. from observa1 Phil. Mag., (6), 18, 229; 1909.

2 Verh. d. V. z. Beford. d. Gewerbfliesses, 83, 403; I904. 
tions made on the whistling arc. His method was in principle the same as Duddell's, but the alternating current was produced by the arc itself, and not by a separate dynamo. The current in a whistling arc is equivalent to a small alternating current superimposed on a direct current. He says concerning it that "only about oneeighth of the observed potential difference, namely, in this case, about 6 volts, was used in overcoming the ohmic resistance of the arc, while the remainder, namely, about 40 volts, is to be considered a polarized potential difference, or counter E.M.F. (cathode and anode drop in potential)."

We may sum up these results by saying that they show that the potential difference between the terminals of the arc varies when the current is varied in much the same way as the potential difference between the terminals of the cell of a storage battery, but they show nothing, of course, concerning the phenomena occurring after the impressed E.M.F. is removed. For this purpose we must examine the second group of experiments.

Residual E.M.F. - The second method of testing for a counter E.M.F. in the arc was to measure the residual E.M.F. after the impressed E.M.F. was removed. There is no question of the meaning of terms involved in these measurements and there is apparently no great experimental difficulty, and yet there has perhaps been as great disagreement among experimenters concerning such an E.M.F. as concerning any question in physics.

Edlund ${ }^{1}$ not only suggested a counter E.M.F. as explaining the relation which he found between the potential difference at the terminals of the arc and its length, but 1 Pogg. Ann., 134, 250 and 337; 1868. 
endeavored to find a corresponding residual E.M.F. He passed a current from a number of cells through the vapor of the arc after the impressed E.M.F. had been removed, passing it first in the direction of the supposed E.M.F. and then in the opposite direction. He found the current in the first direction to be the greater. To compare the E.M.F.'s in the two cases he eliminated the resistance of the circuit by using Ohm's law, but Ohm's law does not at all apply to the case of discharge through hot vapors, and the supposition in this case introduced a very large error. He was led to believe that the counter E.M.F. was equivalent to Io or I 5 Bunsen cells.

But eight years before this Wild ${ }^{1}$ had measured this same quantity by throwing the arc with a double-throw switch from the batteries to a high resistance galvanometer. He was not able to determine the E.M.F. accurately, because the resistance of the vapor between the carbons was not known to him, but he concluded that it was more than Ioo times as great as the E.M.F. produced by a copperGerman silver couple having the same difference of temperature at its terminals. This statement, indefinite though it is, is more accurate than any statement of residual E.M.F. made for several decades thereafter.

After Edlund's experiments there was a series of observations by different experimenters, giving all kinds of values from zero to two volts. The fact that many of the earlier experimenters found no residual E.M.F. is less surprising, since they were looking for one comparable with the supposed counter E.M.F. of the arc, and we can now say definitely that there is none of this order of magnitude. But that some of the later experimenters should have 1 Pogg. Ann., III, 624; 1860. 
differed so decidedly as to a small residual E.M.F. is hard to understand.

Lecher, ${ }^{1}$ Luggin, ${ }^{2}$ Corbino and Liga $^{3}$ concluded that there was no residual E.M.F. Strenger ${ }^{4}$ concluded that if there was one it was small compared with ro volts. Hertzfeld ${ }^{5}$ thought at first that he had found one, but afterwards concluded that the observed effects were due to thermojunctions outside the arc. Le Roux ${ }^{6}$ found a residual E.M.F. but did not give its magnitude.

Arons $^{7}$ thought that the vapor remaining between the carbons after the impressed E.M.F. was removed was not conducting for small voltages, and that it was necessary to apply I8 volts in order to get any current through it.

Granqvist ${ }^{8}$ showed that Arons' failure to get a current with lower voltages was due to using a non-sensitive galvanometer. Granqvist himself was able to pass a current from one Daniell cell. His method was similar to Edlund's, except that he used a rotating switch which closed the galvanometer circuit 0.0009 second after the main circuit was broken. He also assumed Ohm's law to hold, but his voltages were much smaller than Edlund's, so that the error which was introduced in his calculations was much smaller. He found a residual E.M.F. of 0.23 volt in the opposite direction to that of the impressed E.M.F.

Blondel, ${ }^{9}$ using a method almost identical with that of

1 Wien. Sitzungsber., 95, 2 A, 992; .I887.

2 Wien. Sitzungsber., 98, 2 A, I192; r889.

${ }^{3}$ Monash, Der Elektrische Lichtbogen, p. I34.

4 Wied. Ann., 45, 33; 1892.

5 Wied. Ann., 62, 435; 1897.

- Lum. Elec., 3, 285, and C. R., 92, 709; I881.

7 Wied. Ann., 57, 185; 1896.

8 Beib., 22, 243; 1898.

- Lond. Elec., 39, 6r5; 1897. 
Granqvist, concluded that there was no E.M.F. I/600 second after the arc was interrupted as great as o.I 6 volt. He used both solid and cored carbons, long and short arcs, changed the time between the disconnection of the arc from the main circuit and the connection to the galvanometer circuit, and in other ways varied the conditions as far as possible.

On the other hand Hotchkiss, ${ }^{1}$ using an oscillograph having a period of 0.0002 second, found indications of an E.M.F. This was less than 0.66 volt, but was still clearly discernible. Mitkiewicz ${ }^{2}$ found a residual E.M.F. of from I.5 to 2 volts. Becknell ${ }^{3}$ found an appreciable E.M.F. existing for several seconds after the impressed E.M.F. was removed. He determined the amount by balancing it against a known potential difference by means of a sensitive galvanometer. The values which he found are shown in Fig. I2. When the arc was first extinguished the resistance was small, but increased very rapidly with the time. He showed that the residual E.M.F. was not due to the thermal junctions outside the arc.

It is very difficult to reconcile the work of Blondel with that of the others. Furthermore, it is hard to see how it could be otherwise than that there is such a residual E.M.F. It was shown by Dubs ${ }^{4}$ and Olivette ${ }^{5}$ that the current flows from a cooler carbon to one very hot. This may amount to several volts. All the phenomena observed by Becknell are such as would be expected from the difference in tem-

\footnotetext{
1 Trans. Amer. Phys. Soc., 2; rgor.

2 Science Abs., 7, 360; 1903. Beib., 27, 465; I903.

'Phys. Rev., 21, I8I; I905.

- Centralbl. f. Elektrot., ro, 749; r888.

5 Lond. Elec. Rev., 31, 728; 1892.
} 
perature of the carbons. There is probably a very small area on the cathode which is as hot as the anode, so that when the impressed E.M.F. is first removed the difference in temperature between the electrodes is small. The area of this high temperature on the cathode is small. It will,

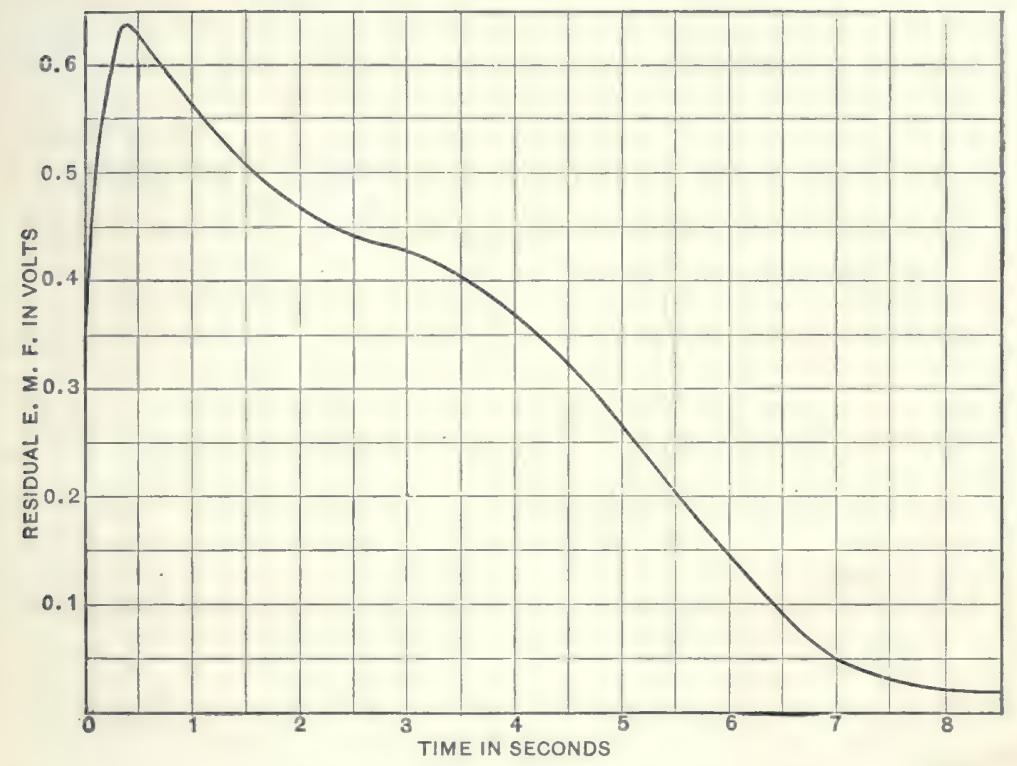

Fig. I 2 .

therefore, cool more quickly than the anode, so that the difference in temperature between the electrodes at first increases. This would correspond to the increase in the residual E.M.F. observed by Becknell. The observed residual E.M.F. is in the direction corresponding with this view, since the anode is hotter than the cathode after the arc is extinguished. 
This explanation can be tested by changing in some way the temperature of the electrodes. The greatest change in the residual E.M.F. may be expected when the anode is cooled, for under ordinary conditions this is hotter than the cathode, and when cooled we should expect the E.M.F. to be diminished or reversed. Such a test was made by myself. ${ }^{1}$ The anode was cooled by three different methods. In the first the anode was a hollow carbon through which water could be passed. In the second a carbon arc was placed in a vacuum, where the anode does not become heated as much as in air. With both of these methods the residual E.M.F. was much smaller than when an ordinary arc between carbons in air is used.

In the third method mercury was used for the anode with the arc in a vacuum. Here the temperature of the anode was lower than in either of the other cases and the residual E.M.F. was reversed. These results were all in harmony with the idea that the residual E.M.F. of the arc is due to the difference in temperature of the terminals.

Temperature of the Arc. - There is at present no method of determining the exact temperature of the arc. The temperature of the vapor is not even known approximately. All that one can say about the vapor is that the most refractory substances may be vaporized in it. Since this is so, the temperature of the vapor must certainly be hotter than that of the carbon electrodes. Moissan ${ }^{2}$ found that with large currents more refractory elements were vaporized than with small, and concluded that with such currents higher temperatures were reached. This cannot be considered proven, since the greater power to vaporize may have

1 Phys. Rev., 30, 311; 19ro.

2 C. R., II6, I429; I893, and II9, 776; I894. 
been due to the greater volume of the hot gas, not to its higher temperature.

We have somewhat more definite knowledge concerning the temperature of the carbon terminals of the arc, and this is what is commonly meant when reference is made to the temperature of the arc. Yet all attempts to find this temperature make use of some assumption which cannot be strictly verified. With one exception all experimenters have made use of observations on the radiation from the carbons and have computed from these the temperature by means of some formula. The majority of these formulæ have been empirical and while they have been found to hold for lower temperatures, there is no proof that they also hold for higher ones. This is certainly true for those used in the earlier work. Thus Becquerel ${ }^{1}$ in I 833 assumed that $L=a\left(\epsilon^{b(T-\theta)}-\mathrm{I}\right)$, where $L$ is the intensity of the red light, $\theta$ the absolute temperature of the surrounding body, $T$ the temperature of the luminous body, and $a$ and $b$ constants depending on the nature of the body. From this he computed the temperature of the arc to be $2070^{\circ} \mathrm{C}$.

Rosetti, ${ }^{2}$ using a somewhat more complicated formula found the temperature of the anode to lie between $2400^{\circ}$ and $2900^{\circ} \mathrm{C}$., and that of the cathode between $2138^{\circ}$ and $2530^{\circ} \mathrm{C}$. Other formulæ were given by Le Chatelier, ${ }^{3}$ Wilson and Gray, ${ }^{4}$ Dewar, ${ }^{5}$ and Petavel. ${ }^{6}$ Their results ranged from the value given above by Becquerel to $6000^{\circ} \mathrm{C}$., the value given by Dewar. This last was com-

1 Ann. de. Chem. et Phys., (3), 68, 49; 1833 .

2 Nuovo Cim., (3), 6, 202; 1879, and 7, 138 and $185 ; 1880$.

3 Journ. de Phys., (3), 1, 185; 1892.

4 Proc. Roy. Soc., 58, 24; 1895.

- Proc. Roy. Soc., 30, 85; I880.

- Phil. Trans., r91 A., 515; 1898. 
puted on the assumption that radiation varies as the square of the temperature, an assumption which is quite incorrect.

Violle ${ }^{1}$ is the only experimenter who has made a determination of the temperature of the arc without making use of the radiation from it. He allowed small parts of the heated anode to fall into a calorimeter and measured the heat which they gave out. Assuming that the specific heat of the carbon is the same at the higher temperatures as it is at the lower, the temperature of the arc was computed from this amount of heat. This assumption is as reasonable as any made in attempting to solve this problem, but the experimental difficulties of this method are very great. When one remembers that only small pieces of the carbon can be used and that only one surface of these can have the temperature of the anode, it is surprising that Violle's results were as similar to those found by more recent methods as they are. By this method he computed the temperature of the anode crater to be $3600^{\circ} \mathrm{C}$.

During the last few years there has been a great advance in our knowledge of the radiation from "black bodies." Such a body would be one which absorbs completely radiations of all lengths which impinge upon it. There are four equations concerning the radiation from such a body which have been deduced from theoretical considerations: the Stefan-Boltzmann law, i.e., $E=c T^{4}$, where $E$ is the total radiation from the black body, $T$ its absolute temperature and $c$ a constant; Wien's displacement law, i.e., $\lambda_{m} T=$ const., where $\lambda_{m}$ is the wave length having the maximum amount of energy; Wien's law for the distribution of energy in the spectrum from a black body, i.e.,

${ }^{1}$ C. R., II5, I273; I892. Journ. de Phys., (3), 2, 545; I89.3. 
$J=c_{1} \lambda^{-5} \epsilon^{-\frac{c_{2}}{\lambda T}}$, where $J$ is the amount of energy of any given wave length, $\lambda$ the wave length, and $c_{1}$ and $c_{2}$ constants; and Planck's modification of Wien's law, i.e., $J=c_{1} \frac{\lambda^{-5}}{\epsilon^{\frac{c_{2}}{\lambda t}}-\mathrm{I}}$. This last has been found to hold through

a more extended range of temperature than that of Wien's, but for wave lengths in the visible spectrum Wien's law applies with sufficient precision.

Although these equations have been proven only for ideal black bodies, carbon comes sufficiently near to being such a body to enable us to make use of them in getting an approximate value for the temperature of the arc. The first of the equations was used by Fery ${ }^{1}$ and the value which he gave to the temperature was $3490^{\circ} \mathrm{C}$.

Lummer and Pringsheim used the second formula. They were led to believe that the radiations from the carbon of the arc were intermediate between those from platinum and those from a black body. From this they computed the temperature of the arc to lie between $375^{\circ}$ and 4200 degrees absolute temperature. Very ${ }^{2}$ applying this same method to data given by Abey and Festing ${ }^{3}$ gave the temperature of the arc as between 3600 and 4000 degrees absolute.

Wanner, using the third formula, found the temperature to vary from 3700 to 3900 degrees absolute, depending on the kind of carbon used. Fery using the same method gave a much higher value. Waidner and Burgess ${ }^{4}$ using

1 C. R., I34, 977 and I201; I902.

2 Astrophys. Journ., 10, 209; 1899.

* Proc. Roy. Soc., 35, 334; I883.

4 Phys. Rev., I9, 241; 1904. Bull. of Bureau of Standards, I, I I3; I904. 
three different kinds of pyrometers in connection with the same formula found values varying from 3860 to 3720 degrees absolute. A comparison of these results is given in the following table, which is taken from the article by Waidner and Burgess.

\begin{tabular}{|c|c|c|}
\hline Observer. & $\begin{array}{l}\text { Absolute tem- } \\
\text { perature of } \\
\text { the arc. }\end{array}$ & Method. \\
\hline $\begin{array}{l}\text { Le Chatelier....... } \\
\text { Violle............ }\end{array}$ & $\begin{array}{l}\text { degrees } \\
4370 \\
3870\end{array}$ & $\begin{array}{l}\text { Photometric; intensity of red light. } \\
\text { Calorimetric; specific heat of carbon. }\end{array}$ \\
\hline Wilson and Gray. . & 3600 & $\begin{array}{l}\text { Total radiation of copper oxide, empirical rela } \\
\text { tion for. }\end{array}$ \\
\hline Wanner. & $03700-3900$ & $\begin{array}{l}\text { (Varying with carbon used.) Photometric in } \\
\text { terms of Wien's law. }\end{array}$ \\
\hline Very......... & Between & $\begin{array}{l}\text { Wave length of maximum energy, Wien's dis } \\
\text { placement law; } \lambda T=C \text {. }\end{array}$ \\
\hline $\begin{array}{l}\text { Lummer and } \\
\text { Pringsheim. }\end{array}$ & $\begin{array}{l}\text { Between } \\
3750 \text { and } 4200\end{array}$ & $\begin{array}{l}\text { Wave length of maximum energy, Wien's dis- } \\
\text { placement law; } \lambda_{m} T=C \text {. }\end{array}$ \\
\hline Fery............ & $\begin{array}{l}a 3760 \\
a 4150\end{array}$ & $\begin{array}{l}\text { Total Radiation; Stefan-Boltzmann law. } \\
\text { Photometric; Wien's law. }\end{array}$ \\
\hline Waidner and Burgess . & $\begin{array}{r}3690 \\
b 3680 \\
3720\end{array}$ & $\left.\begin{array}{l}\text { Holborn-Kurlbaum pyrometer. } \\
\text { Wanner pyrometer. } \\
\text { Le Chatelier pyrometer. }\end{array}\right\}$ Wien's law. \\
\hline
\end{tabular}

a Black body temperature.

$b$ Pure graphite gives a temperature not over 50 degrees higher.

They say concerning this: "From this table it will be seen that the photometric methods based on the extrapolation of Wien's equation show that the 'black body temperature of the arc' (pure graphite) is at least $375^{\circ}$ degrees absolute, so that its true temperature must be higher than this; it is not possible to state how much, in the absence of more definite knowledge concerning the departure of carbon from black body radiation. In the light of the best evidence that is at present available it would seem that the true temperature of the hottest part of the positive carbon is between 3900 and 4000 degrees absolute."

Variation in the Temperature of the Arc. - There has been a difference of opinion among different experimenters 
as to whether the temperature of the carbon terminals is the same under all conditions. Some have believed that it is the temperature at which carbon vaporizes and consequently is always the same. Others have believed that they have found changes in the temperature. Wilson ${ }^{1}$ examined the luminosity of the anode with different pressures of the surrounding gas and found that with higher pressures it was less luminous. At 20 atmospheres it had fallen to a dull red. He concluded that the temperature of the crater depended on how much it was cooled by the surrounding gas.

Later experiments by Wilson and Fitzgerald ${ }^{2}$ led them to believe that this decrease in the luminosity was due to the formation of $\mathrm{NO}_{2}$, which became so abundant at the higher temperatures as to decrease the amount of light appreciably. They were, therefore, unable to tell whether the temperature of the carbons decreased or not.

Waidner and Burgess in the course of their work varied the current from $\mathrm{I}_{5}$ to 30 amperes, and concluded that the temperature of the positive anode increased about 70 degrees.

On the other hand Reich ${ }^{3}$ using a Wanner pyrometer found the temperature of the cathode to be 3I4O degrees absolute, with currents varying from 3 to I 2 amperes. He found the temperature of the anode to be 3700 degrees absolute, which was also independent of the current. He believed that the change observed by Waidner and Burgess may have been due to the increased brilliancy of the gases in the arc with larger currents. He found, however, that

1 Proc. Roy. Soc., 58, I74; I895.

2 Proc. Roy. Soc., 60, 377; 1897.

${ }^{3}$ Phys. ZS., 7, 73; 1906. 
sudden changes in the current produced a decided effect on the cathode. A sudden decrease in the current caused the cathode to become dimmer, but it quickly became hot again, although smaller in extent.

An attempt was made by myself ${ }^{1}$ to find the temperature of the vapor of a carbon arc in a vacuum, but nothing more definite was determined than that the temperature with as low a pressure as $0.5 \mathrm{~mm}$. was high enough to melt a fine platinum wire. ${ }^{2}$

Effect Produced by Cooling the Electrodes. - There has been much discussion among experimenters as to whether higher voltages are required with cooled electrodes or with those not cooled. Since this has some bearing on the theory of the arc it is a matter of importance. We may, however, pass over the work of the earlier experimenters, since they seem to have worked without due consideration of the conditions. For example de la Rive $^{3}$ attempted to heat the arc by placing an alcohol lamp under it. There would be three results produced by this experiment other than the one which he had in mind. First, the flame would probably cool the carbons of the arc instead of heating them. Secondly, the flame would blow the arc out of its original shape, and thus cause a greater drop in potential through it. Lastly, the gases of the flame would change the character of the arc and this might have a very appreciable effect on the voltage.

Again Lecher ${ }^{4}$ cooled one of the electrodes of the arc by immersing it in a mercury bath which was in turn kept

1 Phys. Rev., I9, 123; I904.

2 The temperature of the mercury arc will be considered in connection with the other experiments on that arc.

s Archive de l'Electricité, I, 262; I84 I.

4 Wien. Sitzungsber., 95, 2 A, 992; 1887. 
cool by running water, and concluded that cooling the electrode decreased the amount of voltage required. It has somewhat recently been shown by myself ${ }^{1}$ that this decrease was caused by vapor from the mercury passing into the arc and that when this did not occur the voltage increased. Indeed several experiments which were supposed to show that cooling the electrode lowered the voltage were repeated by myself, and when proper care was taken it was found that in every case cooling the electrodes raised the required voltage.

This is also what one would expect from a consideration of the phenomena bearing indirectly on the subject. For example, if the terminals of the arc are separated for some distance when it is first started, the arc is more apt to go out than it is, if the terminals are allowed to become hot before being separated. If then a longer arc can be maintained when the carbons are hot, one would conclude that heating the terminals allows the current to flow with a smaller potential difference. With an alternatingcurrent arc it requires a higher voltage for a given current when the current is increasing than when it is decreasing. When it is increasing, the arc has been cooled and has not yet attained its maximum temperature. When it is decreasing, it has been hot and is cooling, so that with the same current the electrodes are cooler in the first place than in the second.

Again it is much more difficult to maintain an arc in hydrogen than in air, and this has generally been explained as being due to the fact that hydrogen cools the electrodes more rapidly than air, as if cooling the electrodes made necessary a greater potential difference.

1 Phys. Rev., 30, 315; 1910. 
Probably the most satisfactory method of showing the effect which cooling the electrode has, is to pass an arc between copper or carbon tubes and to observe the voltage first when the tubes are hot and second when they are somewhat cooled by passing water through the interior of the tubes. This method has been used by Tommasi, ${ }^{1}$ Schultze, ${ }^{2}$ Malcolm and Simon, ${ }^{3}$ and myself ${ }^{4}$ and all have found that with cooled electrodes higher voltages are required. ${ }^{5}$

The changes in the anode and cathode drops were also examined by myself, and it was found that the greater part of the change in voltage was due to a change in the anode drop. The change in the different parts of the arc is roughly proportional to the potential difference existing in those parts before the cathode is cooled.

Size of the Anode Crater. - It is well known that the size of the anode crater increases as the current is increased, but there seems to be no agreement as to exactly how large it is, nor what the exact relation is between its area and the current. Andrews ${ }^{6}$ found the area of the crater to be directly proportional to the current. Mrs. Ayrton, using cored carbons, found it to be a linear function of the current, but not quite proportional to it. ${ }^{7}$ She states that with a current of 4 amperes the diameter was

${ }^{1}$ C. R., 93, 716; I88I.

2 Ann. d. Phys., 12, 837; 1903.

${ }^{3}$ Phys. ZS., 8, 478; 1907.

1 Phys. Rev., 30, 310; I910.

${ }^{5}$ Other experiments were performed by the following:

Cross and Shepard, Proc. Amer. Acad. Sc., 22, 227; 1885.

Mitkiewicz, Beib., 29, 739; 1904.

Stark and Cassuto, Phys. ZS., 5, 265; I904.

'Journ. Tel. Eng., 9, 20I; r880.

7 Mrs. Ayrton's "Electric Arc," p. I5 I. 
3.I $\mathrm{mm}$. and with 20 amperes $6.4 \mathrm{~mm}$. She also found the diameter to increase slightly as the length of the arc was increased.

Granqvist ${ }^{1}$ gives the following formula for the crater with solid carbons, $r=0.043+0.008 I$, where $r$ is the radius in centimeters and $I$ is the current in amperes. As will be noticed, these results are very different from those given by Mrs. Ayrton for cored carbons.

Reich ${ }^{2}$ found that the bright spot on the cathode, which he calls the negative crater, was larger, the shorter the arc. With an arc $9 \mathrm{~mm}$. long the following formula applied: $r=0.01+0.009 I$ centimeters.

Enclosed Arc. - With the common arc in air each of the carbons is consumed at the rate of about one inch per hour. This loss is principally due to oxidation of the carbon. The simplest means of obviating this is to place the arc in an air-tight globe, but it has been found that if it is completely air-tight, carbon vapors and hydrocarbon compounds diffuse to the side of the globe making it more or less opaque. It was found, however, by Marks ${ }^{3}$ that if the arc is one with small current and sufficient length, so as to require a comparatively high voltage, it is possible to admit air in such small amounts that the rate of combustion of the carbons is greatly reduced, while there is still enough air to oxidize the carbon vapors.

With the lamp first made by Marks there was a valve which allowed a small amount of air to enter. With improved carbons this has not been found necessary. There is usually some chance for the leakage of air in the space

1 Phys. ZS., 7, 79; 1906.

2 Phys. ZS., 7, 73; 1906.

Lond. Elec., 3r, 502; r893, and 38, 6r5; r897. 
between the movable carbon and the insulator about it, and this is sufficient to allow the necessary amount of air to enter.

The ends of the carbons with the enclosed arc remain more nearly flat, the crater of the anode being less depressed, and the cathode point being less prominent. The resulting distribution of light is somewhat different from that given by the open-carbon arc.

The great advantage of this form of arc is that the carbons do not need to be renewed nearly as often as with the open arc. This is more of an advantage in this country where the cost of labor is higher than in Europe. As a result it has been used very largely here, and very little in places where labor is cheap.

Characteristic curves of these arcs do not seem to have been published, but it may be said that in general the voltage of an enclosed arc is higher than that for an open arc of the same length and current between the same carbons: As an example of this it was found that the voltage of the arc in a commercial lamp using 6.5 amperes and having a length of $8 \mathrm{~mm}$. was 80 volts.

Miscellaneous. - There are a few more or less isolated facts concerning the carbon arc which should be mentioned before passing to other topics. Such, for example, is the fact that an arc is deflected when in a magnetic field. The first mention of this seems to have been made by Davy in $182 \mathrm{I}^{1}$ and Walker ${ }^{2}$ states that the arc will rotate if one of the electrodes is a steel magnet. Blondel ${ }^{3}$ took photographs of the alternating-current arc in a mag-

1 Phil. Trans., p. 428, 1821 .

2 Pogg. Ann., 54, 514; I84I.

${ }^{3}$ Lum. Elec., 43, 54; 1892. 
netic field and found that it was deflected alternately, as if it was wire which was carrying current.

In 1878 Wilde $^{1}$ showed that the reason an arc between two parallel carbons travels away from the ends at which the current enters the carbons is that the magnetic action of the current upon itself forces it in this direction. Somewhat recently Morris ${ }^{2}$ examined, by means of an oscillograph, the current through an arc just before it was broken by being deflected by a magnet and showed that it was a pulsating current. This accounts for the shriek which the arc gives when it is thus extinguished. ${ }^{3}$

While making photometric measurements with rotating sectors, Trotter ${ }^{4}$ discovered that there is a bright spot near the middle of the crater which rotates with frequencies varying from 50 to $45^{\circ}$ per second, the most common being about roo per second. This was most noticeable with short humming arcs. The direction of rotation varied without any known cause.

Dewar ${ }^{5}$ showed that the vapors of the arc exert a pressure on the electrodes, that on the anode being the greater. This has been verified by Cheneveau. ${ }^{6}$

1 Nature, 19, $152 ; 1878$.

${ }^{2}$ Lond. Elec., 59, 707; 1907.

${ }^{3}$ Other experiments with an arc in a magnetic field have been performed by the following:

Cassellman, Pogg. Ann., 63, 589; 1844 .

De la Rive, Phil. Trans., part I, 37; I847.

Quet, C. R., 34, 805; I821.

Joubert, C. R., 9I, 16r; 1880.

Martiny, Dissertation Rostock and Ber. d. Phys. Ges., 2, I99; I904.

Child, Phys. Rev., 20, 100; I905, and 24, 498; I907.

4 Lond. Elec., 33, 297; 1894.

'5 Chem. News, 45, 37; 1882. Proc. Roy. Soc., 33, 262; 1882.

- Ecl. Elec., 20, 402; 1899. 
The current between a hot carbon used as cathode and a cooler one used as anode with different temperatures and different potential differences has been examined by Pollock and Ranchaud. ${ }^{1}$ They studied this with especial reference to the conditions under which the current changes from a small, non-luminous discharge to the arc. The hot carbon was heated by passing an electric current through it, and its temperature was determined by a Holborn-Kurlbaum optical pyrometer. The anode became heated somewhat by the radiation from the cathode and its temperature was determined by a thermojunction.

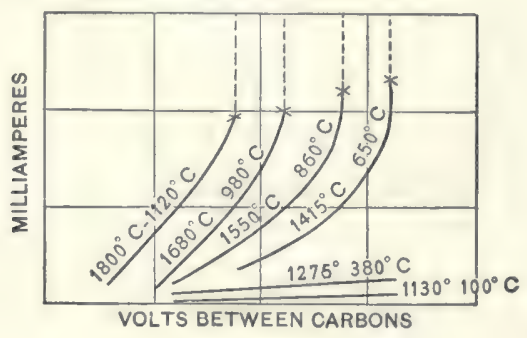

FIG. I3.

Some of their results are given in Fig. 13. The first number above each curve gives the temperature of the cathode and the second one that of the anode. The dotted parts of the curve are extrapolated. The crosses " $\mathrm{x}$ " indicate the points at which the discharge became an arc.

1 Phil. Mag., (6), 17, 366; 1909. 


\section{CHAPTER III.}

Arc in Air Between Other Substances than Carbon.

So far we have considered only arcs between terminals of nearly pure carbon. As has been stated it is possible to use for terminals many other substances. Attempts were made in the early part of the last century either to use a metal or to add some substance to the carbon which would increase the efficiency of the arc for giving light. These attempts did not at the time meet with success. They led rather to the belief that the substance which could be raised to the highest temperature without being melted was the most efficient, and since carbon can be raised to a higher temperature than any other material which is commercially feasible, it was believed to be the best for electrodes.

But the discoveries of Auer and Nernst showed that there are materials which have an emissive power more or less selective and that an ideal black body is not necessarily the best for emitting light. It, therefore, became evident that there might be some better substance which could be used for the electrodes of the arc. Several of those which have since been tried have met with a large degree of success. There are now three types of arcs which embody some improvement over the carbon arc. These are arcs having electrodes which are principally carbon, but have also a mixture of other substances, such as the "flaming" arcs; arcs having an oxide for one of the 
electrodes, of which the magnetite arc is the best known; and arcs between metals, of which the mercury arc in a vacuum is the only commercial example.

All of these arcs require a lower voltage for the same current and length than the carbon arc, and they all give more light for the same amount of energy. It is also true that each has some disadvantage. Many compounds which might be introduced into the arc give fumes which make them disagreeable in an enclosed room, and some blacken any globe which may surround the arc. Many give colors which are better suited for advertising purposes than for common use. Some combinations of salts give trouble either with regulating the lamp, or with starting the arc after it has been extinguished.

Unfortunately investigation of these arcs must be entirely empirical, for we know nothing of the laws of radiation from mineral vapors at high temperatures, nor of the laws governing the relation between electrical excitation of an atom and its emission of light. We can not reason out in advance the amount of light to be expected from a given substance. An analysis of the spectrum of different vapors does not always help us. The spectrum of calcium fluoride, for example, has no resemblance to that of other salts of calcium and is more brilliant.

Flaming Arc. - The general appearance of the first of these types, namely, those whose electrodes are carbon with certain salts mixed with them, is quite different from that of the arc between pure carbons. This is indicated by Fig. I4, which is reproduced from a photograph of a "flaming arc," as this class of arcs is called. This arc is very much longer than the older form, and the greater part of the light comes from the vapor instead of from the carbon. 
The first mention which I have found of the arc between carbons in which other substances were mixed is that in an article by Casselmann ${ }^{1}$ who tried the effect of

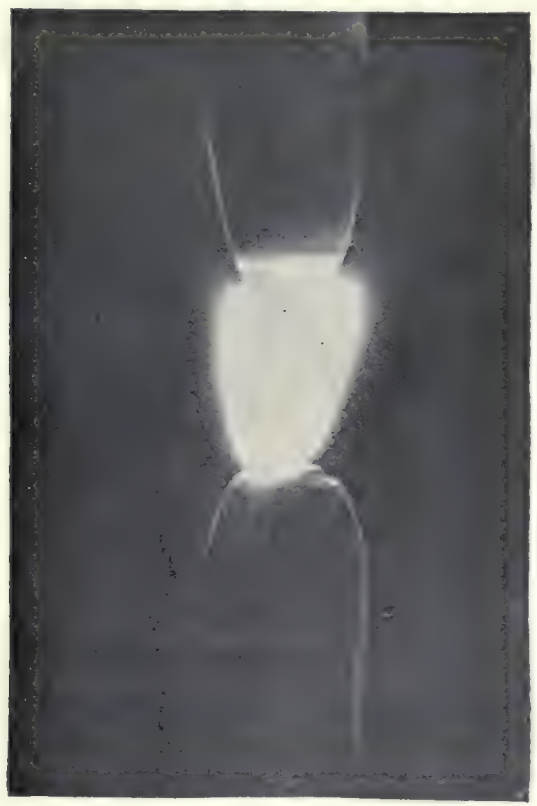

Fig. I4.

introducing different salts into the arc and found that they allowed longer arcs with the same number of cells. He found that strontium nitrate, copper nitrate, and zinc chloride were especially helpful in producing luminosity. ${ }^{2}$

1 Pogg. Ann., 63, 576; 1844 .

2 Other investigations were made by the following:

Archereaus, C. R., 84, $137 ; 187 \%$.

Carre, C. R., 84, 346; 1877 .

Luggin, Centralbl. f. Elektrot., 10, 567; 1888. 
The first commercial success in this direction was made by Bremen who discovered the advantage of using calcium fluoride. This is the basis of nearly all the combinations of salts used for flaming arcs. Other salts are added to a greater or less extent to modify the kind of light, or to avoid some of the disadvantages mentioned above. Thus calcium phosphate is often added, especially for direct-current arcs. According to Mahlke ${ }^{1}$ cerium chloride forms two-thirds of the salts used for white flaming arcs, the other third being chiefly calcium salts.

The carbon for these lamps consists of an outer cylinder made of hard carbon and an inner core made of a mixture of carbon and the salts to be used. The outer cylinder protects the salts from too rapid consumption and gives the necessary conductivity to the electrode. The inner core is made of a finely divided carbon, usually lampblack, mixed with the salt, which must be in the form of a powder. If the salt is not thoroughly mixed with the carbon, they will produce miniature explosions and uneven burning of the arc.

According to Mahlke the core consists of 40 per cent of lampblack and 60 per cent of metallic salt. There are, however, few of the flaming-arc carbons now on the market which have as high a percentage of salt as this.

The resistance of these electrodes is higher than that of solid carbons, and if they are long, much energy is wasted in forcing the current through them. To remedy this, wires are usually run through the length of the electrodes to serve as conductors. These wires vary from onehalf to three-fourths of a millimeter in diameter. Zinc, brass and tin have been used for this purpose.

1 Elec. World, 57, 675; I9rr. 
The great advantage of these arcs is that they give much more light for the power consumed than carbon arcs. They also have two disadvantages. One of these is the color of the light given. With the "white" flaming-arc carbons now on the market this difficulty has been largely" obviated, the light from these being nearly the same as that from common carbons. However, what is thus gained in color is lost to some extent in efficiency, the watts per candle for the "white" flaming arc being somewhat greater than those for the "yellow" flaming arc.

The chief disadvantage of the flaming arc is that the carbons are more rapidly consumed than those of the common carbon arc, and very much more rapidly than those of the enclosed arc, and require more frequent renewal. For this reason the flaming arc has not been widely used in this country where the cost of labor is high. In Europe they have been very extensively used.

Considerable progress is, however, being made in obviating this difficulty by special forms of lamps. In many of these the air is partially excluded, as in the enclosed arcs, and ventilating flues which keep the solid products of combustion from being deposited on the surrounding globe are provided. The life of the electrodes has by this means been increased to 80 or even roo hours.

The life of the electrodes can be increased by making either the electrodes longer or their diameters greater. Increase in length, however, leads to an unsightly and clumsy lamp, while increase in diameter leads to unsteadiness. Increased life can also be attained by using carbons which approach in composition more nearly to the common carbon, but such increase leads to a decided loss in efficiency. The potential difference needed to maintain the flaming 
arc is much less than that needed for carbon arcs. Scarcely any measurements have been published regarding this, but a few observations have been recently made by my-

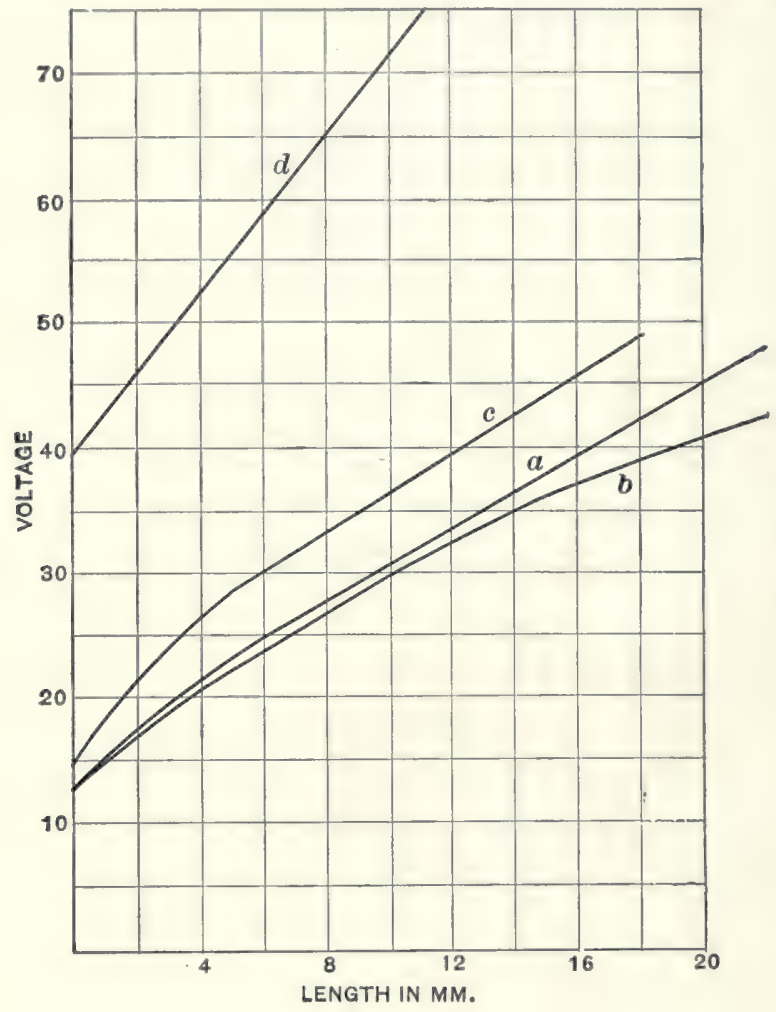

FIG. 15 .

self and are given in Fig. I5. With different settings for length the readings of the voltmeter varied by one or two volts, but by taking several observations an average was found which represents closely the correct value.

Curve $a$ in Fig. I5 represents the voltages for different 
lengths with what is known as the "white" flaming arc. The current was kept constant at io amperes. For comparison the corresponding curve for the open carbon arc as plotted from Mrs. Ayrton's equation is given in curve $d$. The great difference between the two forms of arc is very evident.

The data plotted in curve $a$ were taken with the open flaming arc having the carbons vertical, the positive one being below. In general the flaming arc is enclosed in some kind of a globe and usually with some arrangement to produce a draft which will keep the ash from settling on the globe. This curve, therefore, does not give the exact voltage which would be needed when running under commercial conditions. It does, however, give an approximate idea of such voltage.

Observations were also made of the voltage with the flaming arc enclosed in a globe which was very nearly airtight. The data thus taken are plotted in curve $b$.

Observations were made with carbons which were purchased from several different dealers and were supposed to be different kinds of carbons. With one exception they showed but slight differences as far as the potential difference at the terminals was concerned, so that the curves given in Fig. I 5 would apply fairly well to nearly all of the carbons. The one exception gave higher voltages with the longer arcs. For example, with an arc $22 \mathrm{~mm}$. long the voltage was $5^{\mathrm{I}}$ instead of 47 volts.

The voltages required for the "yellow" flaming arc are slightly less than those of the white arc. Such an arc is represented by the curve $c$.

In Fig. I6 there is given a curve showing the voltages with different currents, the length being constant at Io $\mathrm{mm}$. 
This curve represents the voltages with the white arc. Those with the yellow arc were only slightly less than these. This curve differs from those given by Mrs. Ayrton for the carbon arc in that it shows an increase with the larger currents. This was apparently due to the tendency of the arc with the larger currents to flare out to one side. It was very difficult with the currents of more than 25 amperes to get a steady condition and different readings

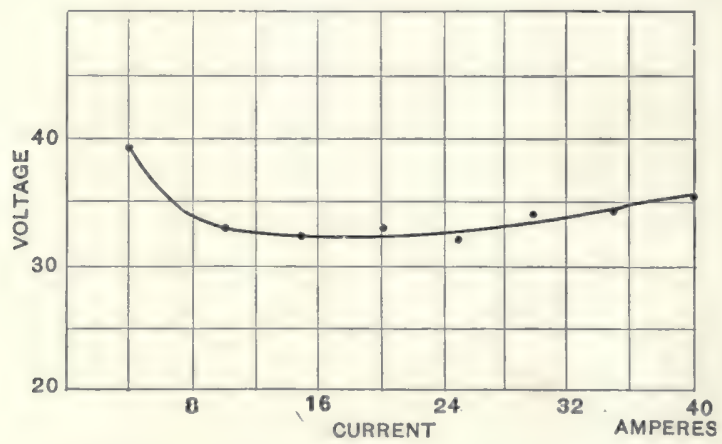

FIG. I6.

varied by 3 or 4 volts. But the voltage with the large currents appeared even with the most steady conditions to be greater than those required for 20 amperes.

The small voltage required for the flaming arc is largely due to the small drop in potential at the anode. The decrease in the anode drop caused by putting salts in the arc was shown several years ago by Luggin $^{1}$ who found that sprinkling the carbons with soda diminished the anode drop to less than one-half of its former value.

In the examination of flaming arcs made by myself it ${ }^{1}$ Centralbl. f. Elektrot., ro, 567; 1888. 
was found that the drop between the anode and carbon pencil I $\mathrm{mm}$. in diameter placed as close to the anode as possible without touching it was $\mathrm{I} 2$ volts. The drop between such a pencil and the cathode was 8.5 volts. These observations were made with open $\operatorname{arcs}$ I $\mathrm{cm}$. long and having a current of ro amperes. The values were found to be nearly independent of the length of arc or the current and were the same for several different makes of flamingarc carbons. The anode drop with arcs of the same length and current between solid carbons is 35 volts (p. I4) which is approximately three times that for the flaming arcs, while the cathode drop is nearly the same for both kinds of carbons.

The electric force through the arc can be found approximately by getting the rate of change of the potential difference between the terminals of the arc with respect to the length. The data on the preceding page show that this rate of change was approximately 1.65 volts per millimeter for the open flaming arc when the current was ro amperes, while with the arc between solid carbons the value of this quantity is 3.12 volts per millimeter.

Arc between Metals. - With the exception of the arc between mercury terminals in a vacuum none of the arcs between metals have proven to be of commercial value, for the metals are consumed much more rapidly than carbon and the arcs between them sputter continually and give much less light than carbon arcs. Many investigations have, however, been made for the purpose of learning how such arcs behave. The earlier of these were of less value because one can not be sure whether the experimenter was using an arc or only a spark. However, there 
were a few facts clearly shown in the early part of the last century. For example, it was shown by Davy ${ }^{1}$ that carbon terminals give out more light than any of the metals. It was shown by Metteucci ${ }^{2}$ that the conductivity of the arc between copper terminals was more than that between some of the other metals.

Somewhat later it was shown by Edlund ${ }^{3}$ that there was no measurable conductivity between silver terminals $\mathrm{I} / 8 \mathrm{o}$ sec. after the arc had been extinguished, although with carbon terminals there was a very appreciable amount of conductivity. It has since been shown that all arcs between metals lose their conductivity very rapidly after the removal of the impressed E.M.F. ${ }^{4}$

It is only in recent years that measurements have been made on the relation between potential difference, current and length of metal arcs. These measurements show that the metal arcs require a much smaller voltage than the carbon arcs for the same current and length of arc and that the characteristic curves are quite similar to those for the carbon arc, but the fact which they most clearly show is that different experimenters working under different conditions arrive at very different results. This is seen from the following formulas which have been given for this relation for the arc between copper terminals. In all of these $E$ represents the potential difference in volts, $I$ the current in amperes and $l$ the length of the arc in millimeters.

1. Journ. Roy. Inst., I, 166; 1802.

2 C. R., 30, 20I; 1850.

8 Pogg. Ann., 134, 250; 1868.

- See also the following :

De la Rive, Phil. Trans., part 2, 3r; 1847.

Arons, Wied. Ann., 57, 197; 1896. 


$$
\begin{aligned}
& { }^{1} E=26, \text { for } I=5 \text { amperes and } l=2 \mathrm{~mm} . \\
& { }^{2} E=23.86+.67 l I . \\
& { }^{3} E=18.88+22.4 l-3.9 l . \\
& { }^{4} E=21.38+3.03 l+\frac{10.69+\mathrm{I} 5.24 l}{I} . \\
& { }^{5} E=18+\mathrm{I}_{7} \cdot .5+\frac{52.1+3.43}{I} . \\
& { }^{6} E=55+12.5 l+\frac{90 l}{I} .
\end{aligned}
$$

The second of these equations should probably not be considered at all, since other experimenters have shown conclusively that the voltage does not increase with increasing current, but the others are sufficient to show that the voltage is not only a function of the current and length of arc, but also of other conditions. Some of the conditions under which these formulas were found may be indicated by the following. The third formula is only true for a horizontal arc of 2 amperes, the size of the electrodes not being given. The fourth is with rods $16 \mathrm{~mm}$. in diameter with length of arc $2 \mathrm{~mm}$. or less. The fifth is with rods three-eighths inch in diameter with the anode above, while the sixth is for water-cooled electrodes with small currents.

In addition to these results we have the statement of other experimenters that the curve for the arc between copper terminals does not follow any simple formula.

1 Lecher, Wien. Sitzungsber., 95, 2A, 992; I887.

2 Von Lang, Wied. Ann., 31, 39r; r887.

${ }^{3}$ Freedman, Elec. Rev., 37, 253; I895.

4 Guy and Zebrikoff, C. R., 145, I69; I907.

s Upson, Phil. Mag., (6), I4, I40; 1908.

B Grau and Russ, Phys. ZS., 9, 108; 1908. 
Thus, according to Malcolm and Simon, ${ }^{1}$ who worked with small currents, the product $E I$ is not a linear function of $I$, as it is in the preceding equations. They found the potential difference for an arc between copper terminals to vary from 80 volts for 0.6 ampere to 58 volts for I.I amperes, the length in both cases being $4 \mathrm{~mm}$.

Hagenbach and Veillon ${ }^{2}$ found it difficult to make any exact measurements of the copper arc, but, as far as they could, they determined its voltage both in air and in a vacuum, and in no case did the curve follow the Ayrton formula.

There are, however, two facts upon which there is agreement; first, that the potential difference with arcs between metals is in general smaller than that between carbons, and second that the rate of increase with increasing length of arc is greater than with carbon arcs. From this it would follow that the sum of the anode and cathode drops in the metal arcs is less than in the carbon arc, and that the electric force is greater.

The difficulty of making accurate measurements of the metal arcs is partly due to the fact that there are two or more forms of these arcs, and that under some conditions there is a constant fluctuation from one form to the other. Thus it has been shown by Cady and Arnold ${ }^{3}$ that there are two distinct forms of the arc between iron terminals corresponding somewhat to the quiet and hissing forms of the carbon arc. The first stage occurs with small currents, usually less than 2 amperes. The voltage is in the neighborhood of 75 volts. The gas near the anode is

1 Phys. ZS., 8, 477; 1907.

2 Phys. ZS., II, 833; I9го.

${ }^{3}$ Amer. Jour. Sc., (4), 24, 383; I907. 
non-luminous and the arc is quiet. In passing to the second stage there is a sudden increase in current and decrease in potential difference, the gas near the anode becoming luminous and the arc becoming noisy.

Though there is an apparent similarity between the second stage of this arc and the hissing arc with carbon terminals, the causes of the two phenomena are not the same. The peculiarities of the second stage with the iron arc are apparently due to vaporization of the anode. The anode loses weight much more rapidly and the spectrum of the anode is very plain, while with smaller currents it was scarcely visible. Moreover the effect was shown to exist in nitrogen as well as in air, so that it can not be explained as being due to the presence of oxygen.

It is claimed by Hagenbach and Veillon that there are six distinct forms of the copper arc, that there are three different anode drops which may occur, and that with each of these it is possible to have two different cathode drops. The same phenomenon has been found to a limited extent with other metals, although in general the first stage is absent with metals having a low melting point. $^{1}$

Two attempts have been made to find the anode and cathode drops with the arc between metals in air, but it is very difficult to make accurate measurements. The voltage of such arcs under ordinary conditions is very variable and the introduction of a carbon pencil causes it to fluctuate through even greater ranges. Moreover, with the intro-

1 See also the following:

Cady and Vinal, Amer. Journ. Sc., (4), 28, 89; 1909.

Fabry and Buisson, Journ. d. Phys., (4), 9, 929; 1910.

Hagenbach, Physik ZS., I2, IoI5; Igrr. 
duction of a carbon electrode, the vapor of the arc is changed to a certain extent, so that one can not be certain that the anode drop is the same as it was before this was introduced. As a result the measurements which have been made on the anode and cathode drops can not be considered as more than rough approximations.

The measurements indicate, however, that the relative values of the two drops are quite different in metal arcs from what they are in the carbon arc. In the latter the anode drop is much greater than the cathode drop. In the former the two are approximately the same, as was shown by Lecher ${ }^{1}$ for platinum, iron, aluminum and copper and by myself ${ }^{2}$ for zinc, iron and copper. With graphite terminals in air the anode and cathode drops are much the same as they are with carbon. ${ }^{3}$

With a metal arc in a partial vacuum the measurement of the potential within the arc can be made somewhat more accurately. Banderet ${ }^{4}$ has examined the anode and cathode drops of the different forms of the copper arc when in a vacuum. As has been stated, it is possible to have six different forms. In one of these where the appearance at the anode is somewhat similar to that at the anode with the glow discharge in a Geissler tube he found the anode drop to be 34 volts. In a form which is similar to the carbon arc the drop is 26 volts, while in an intermediate form it is 20 volts. These measurements were taken with a pressure of IIO $\mathrm{mm}$., a current of 5 amperes, and an arc length of $20 \mathrm{~mm}$. With all of these

\footnotetext{
${ }^{1}$ Wied. Ann., 33, 609; 1888.

2 Phys. Rev., 12, I49; I90r.

${ }^{3}$ Phys. Rev., 20, 364; I905.

4 Dissertation Basel, Igra.
} 
the cathode drop is approximately I 3 volts. Modifications of these were also found where the cathode drop is slightly less.

Arc between Oxides. - As has been stated, the discoveries of Auer and Nernst regarding the selective emissive power of certain substances led to a further investigation of the arc between other substances than carbon. Among other substances were oxides similar to those in the Nernst lamp. Rasch investigated these oxides and gives the formula $E=3 \mathrm{I} .35+30 / I$, the length of the arc being I mm. ${ }^{1}$ These oxides are non-conductors at ordinary temperatures, and an auxiliary arc was used to heat them until they became conductors. This arc has not been developed into a commercial form. ${ }^{2}$

Measurements were made by Schultze ${ }^{3}$ of arcs between several oxides of metals, by placing the substance to be examined in a cup hollowed out of a carbon electrode. He found, in general, that the oxide having the lower melting point requires the smaller E.M.F. when used as electrode. With very short lengths of the arc he found the voltage to be independent of the current. With greater length the anode drop increased greatly with decreasing current and the cathode drop increased little or none.

Magnetite Arc. - The most important of the arcs between oxides is the magnetite arc. ${ }^{4}$ In this the cathode is magnetite $\left(\mathrm{Fe}_{3} \mathrm{O}_{4}\right)$ and the anode is copper. Magnetite is used for the cathode because it gives off a vapor which is

1 Elektrot. ZS., 22, 55; 190r.

2 See also Elektrot. ZS., 22, 256, 293, and 373; r9or. Verh. d. D. Physik. Ges., 5, 157; 1903, and 6, 137; 1904.

3 Ann. d. Phys., 12, 828; 1903.

4 Elec. World, 43, 974; I904. Trans. Intern. Cong., St. Louis, 2, 721 ; I904. 
very luminous when in the arc, and copper is used for the anode because it is not rapidly burnt away and does not need to be renewed as often as a carbon anode. The anode is large and the rapid conduction of heat by the copper keeps it at a comparatively low temperature. This arc is very efficient and the electrodes need little attention because of their slow consumption.

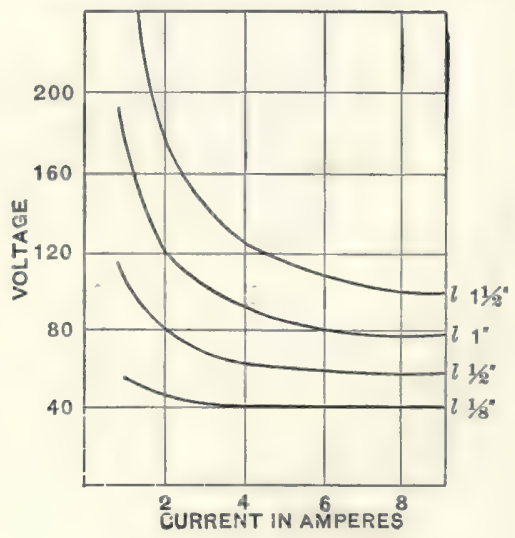

FIG. 17 .

Characteristic curves for this arc are given in Fig. 17 . The equation,

$$
E=33+24.5 l+\frac{25+140 l}{I},
$$

which is of the same general form as those given by Mrs. Ayrton, agrees very closely with these curves.

Dyott ${ }^{1}$ gives characteristic curves for an arc having a copper anode and a cathode consisting of 60 per cent magnetite $\left(\mathrm{Fe}_{3} \mathrm{O}_{4}\right), 27$ per cent titanium oxide $\left(\mathrm{TiO}_{2}\right)$ and I3 per cent chrome iron $\left(\mathrm{FeCr}_{2} \mathrm{O}_{4}\right)$. His values of the volt-

${ }^{1}$ Elec. World, 51, 854; 1908. 
ages for lengths greater than 0.2 inch are in general greater than those for the magnetite arc, while for arcs shorter than this they are less. ${ }^{1}$

A somewhat similar arc is described by Weedon ${ }^{2}$ in which titanium carbide is used for the cathode and copper for the anode. The characteristic curves which he gives for this arc are nearly identical with those given by Steinmetz for the magnetite arc.

Arc between Electrolytes. - As has been stated it requires a very high E.M.F. to produce an arc between a metal and an electrolyte, and when produced it is very unsteady. As far as I have been able to learn, no measurements of current nor potential difference have been taken with such arcs. About all that has been attempted is to show that it is possible to have arcs of this kind. Thus Grove $^{3}$ found that water could be made the anode of an arc and somewhat less easily the cathode, platinum being the other terminal. His source of current was 500 nitricacid cells. ${ }^{4}$

Arc between Unlike Electrodes. - It was noticed by Grove in $1840^{5}$ that the current through an arc is larger, if it passes from a metal which is easily oxidized to platinum than if it passes in the opposite direction. Many years later it was discovered by Sahulka that with an alternating-current arc between cored carbon and iron

1 See also Ladoff, Elec. World, 45, 757; 1905.

2 Paper read at the meeting of the Amer. Chem. Soc., Oct., I gog.

3 Phil. Trans., 142, 88; 1852.

${ }^{4}$ See also the following:

Van der Willigan, Pogg. Ann., 93, 285; 1854.

Stark and Cassuto, Phys. ZS., 5, 264; 1904.

Athanasiadis, Lond. Elec., 61, 873; 1906.

b Phil. Mag., 16, 478; 1840. 
there is an excess of current flowing from the carbon. This called attention again to the phenomenon which had been noticed by Grove and further experiments have shown that in general when the electrodes are of different substances, the current flows more easily in one direction than in the other. ${ }^{3}$ For example, it flows more easily from any metal to carbon than in the reverse direction; more easily from cored carbon to solid than from solid to cored; and more easily from a carbon that is easily kept hot to one that is not, as a rotating carbon disk, than in the opposite direction.

The explanation of this is as follows: The conductivity of the arc depends on the kind of vapor in the arc and also upon the ease with which the cathode can be kept at a high temperature, but does not depend to any large extent on the temperature of the anode. If the anode gives off a conducting vapor when heated, this vapor will affect the conductivity, but the anode does not directly affect the conductivity to more than a very small extent. These facts will be discussed more fully at a later point, but may be accepted for the present in explaining the phenomena under discussion.

If we apply these facts to the arc between iron and carbon, for example, we find that the current flows more easily from iron to carbon; first, because the conductivity of the iron vapor is greater than that of the carbon vapor, and there is more of this vapor sent into the arc

1 See also the following:

Gold, Wien. Sitzungsber., 104, 2A, 814; r895.

Pettinelli, Rend. Acc. Lind., (5), 5, I18 and 136; 1896.

Von Lang, Wied. Ann., 63, I9I; 1897.

Cassuto, Phys. ZS., 5, 263; 1904 . 
when the anode is iron, since there is more heat developed near the anode with arcs in air than near the cathode. That the conductivity of iron vapor is greater than that of carbon is shown by the fact that the drop in potential across an arc having both terminals iron is much less than that about an arc having both terminals carbon.

This part of the explanation of the greater current from iron to carbon was given by Arons, ${ }^{1}$ but the difficulty of keeping the iron hot enough to be the cathode must also be considered. As will be shown in following paragraphs, it is always necessary to have a point on the cathode at a very high temperature. Manifestly, it is harder to keep a spot on iron very hot than one on carbon, first because iron conducts heat more rapidly than carbon, and secondly because iron melts and vaporizes more readily than carbon, and heat is lost in producing these changes. In order to furnish this heat the cathode drop must be large.

The need of this last consideration will perhaps be more apparent in the case of discharge between a stationary carbon rod and a carbon disk kept at a lower temperature by rotating it. Here the current is the greater when the moving carbon is the anode. Arons' explanation is not in this case sufficient, since the vapor in the arc will be carbon vapor, whichever way the current flows. On the other hand, the second consideration applies, for it will clearly be easier to keep the stationary carbon hot than the moving one, so that the current will be larger when the stationary carbon is cathode.

A few observations made by myself on the cathode and anode drops in a carbon-iron arc are quite in harmony with

1 Wied. Ann., 57, 195; 1896. 
this view. The introduction of a carbon pencil into such an arc makes it very unsteady and changes the vapor in the arc to some extent, so that the values given below can not be considered accurate. They are, however, of some value. These observations were made with a constant length and constant current.

\begin{tabular}{|l|l|c|c|}
\hline \multicolumn{1}{|c|}{ Anode. } & Cathode. & $\begin{array}{c}\text { Anode } \\
\text { drop. }\end{array}$ & $\begin{array}{c}\text { Cathode } \\
\text { drop. }\end{array}$ \\
\cline { 1 - 2 } Carbon & Carbon & 38 & 13 \\
Iron & Carbon & 14 & 17 \\
Carbon & Iron & 12 & 21 \\
\hline
\end{tabular}

In the first case both electrodes are carbon. In the second the anode is iron and the vapor from this is highly conducting and causes a large decrease in the drop in potential near the anode. The cathode drop is slightly greater than when both electrodes are carbon, the reason, no doubt, being that the iron anode is cooler than the carbon anode, and allows the cathode to cool off more rapidly, requiring a large cathode drop to maintain the temperature of the cathode.

In the third case there is still enough iron vapor in the arc to cause a small anode drop, but the heat is conducted away from the iron cathode so rapidly that it requires a still higher cathode drop to maintain the temperature of the iron at the required point. 


\section{CHAPTER IV.}

The Arc in Air with Pressures Greater or Less than ONe Atmosphere.

Pressures Greater than One Atmosphere.-But few experiments have been performed with the arc in a gas under pressure. Wilson and Fitzgerald ${ }^{1}$ found that with pressures greater than one atmosphere fumes of $\mathrm{NO}_{2}$ were formed and that these became very plentiful when the

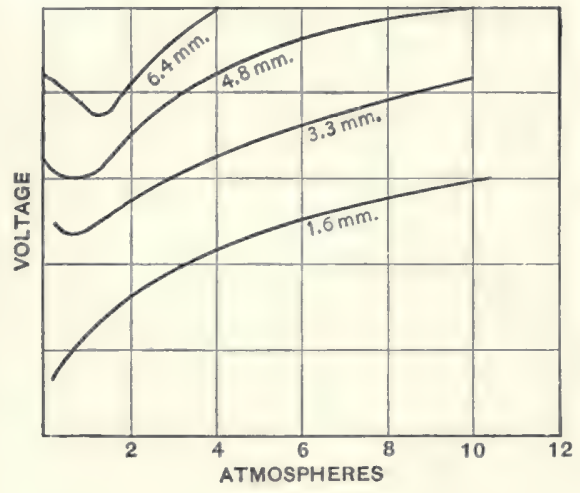

FIG。 I8.

pressure was as great as Ioo pounds per square inch. Duncan, Rowland and Todd ${ }^{2}$ found that the apparent resistance of the arc increased as the pressure was increased, as is shown in Fig. I8. The curves in this give the voltage

${ }^{1}$ Proc. Roy. Soc., 60, 377; 1896.

2 Elektrot. ZS., 14, 603; 1893. 
between the terminals of the arc with different pressures of the gas, the current being maintained at 6 amperes.

Pressures Less than One Atmosphere. - While the arc in a vacuum has no great commercial value, it gives us much information which is useful in forming a theory of the arc, for with very low pressures there are no complications due to chemical actions, the temperature is sufficiently low so that small exploring wires may be used without appreciably distorting the field, and the existence of striations and cathode rays shows the similarity between this form of discharge and those having much smaller currents.

The earliest experiments on an arc in a vacuum were performed before the distinction between arc and spark was made and with many of these experiments it is impossible to determine which form of discharge was really used.

Thus, an experiment was performed by Davy in $1809^{1}$ to ascertain whether any heat sensible to a thermometer is produced by the electric flame in a vacuum, but it can not be definitely stated whether this was an arc or a glow discharge. The same statement must be made of the work by Van Breda. ${ }^{2}$

${ }^{1}$ Mrs. Ayrton's "Electric Arc," p. 25.

2 C. R., 23, 462; 1846.

Nole. - The work of De la Rive (Phil. Trans., I 847 , part I) appears to have been with an arc, while that of De la Rue and Müller (Phil. Trans., I71, A, 65; 1880; Proc. Roy. Soc., 29, 286; 1879) was apparently on a continuous glow discharge, since the current which they used was very small and the voltage several thousand volts. Stenger, in 1885 (Wied. Ann., 25, 3I; 1885), gives four differences between the arc and the glow discharge in a vacuum, but only one of these, namely, that the gaseous portion has less resistance in the arc than in the glow discharge, can be said to be correct, while some of the more important differences were not noted. 
Carbon Arc in a Vacuum. - In more recent work we find more definite statements about the conditions and we can be sure of the form of discharge being used. The greater part of this work has been with carbon electrodes. When the pressure about such an arc is dimin-

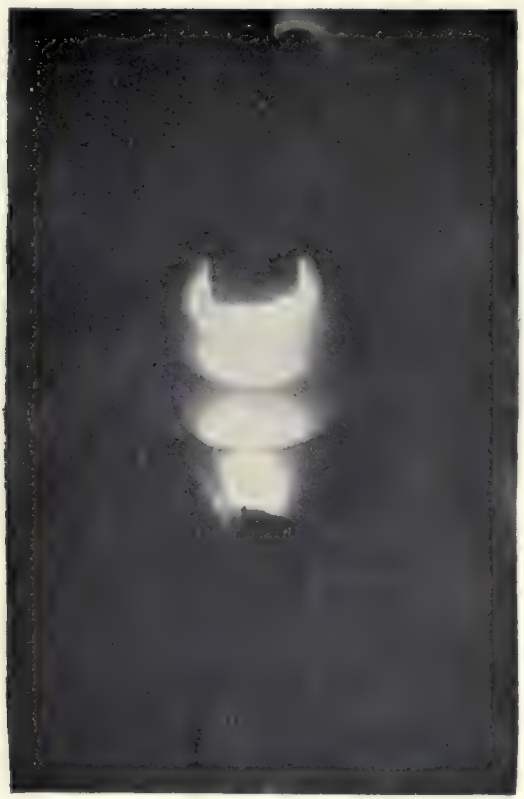

Fig. I9.

ished, the anode becomes slightly less luminous and the cross section of the luminous gas becomes somewhat greater, but the appearance is not greatly changed until a pressure of only a few millimeters is reached. At that point a very decided change occurs. Under certain conditions striations appear, as shown in Fig. I9. As the 
pressure is diminished the number of striations becomes less, as is shown in Fig. 20. With still lower pressures the striations disappear and the glow at the anode moves about in a very erratic way. At times it appears as a ring about the anode a few centimeters above the end, as

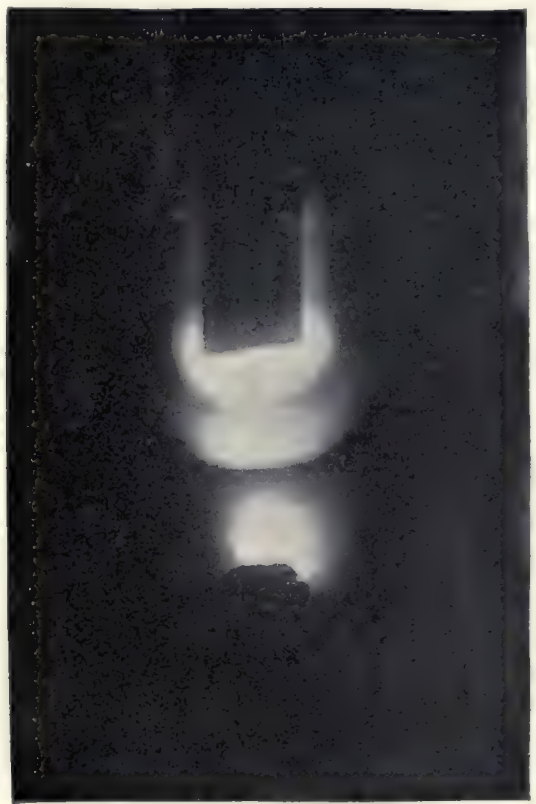

FIG. 20.

in Fig. 2r. Occasionally two rings of this kind have been noticed.

With pressures less than $5 \mathrm{~mm}$. this ring also disappears and the arc consists of a bright spot on the cathode from which cathode rays appear to come and a faint luminosity in the space through which these rays happen to 
stream. When this state is reached the arc is not appreciably affected by varying the distance between the electrodes.

The voltage required to maintain an arc in a vacuum is much less than that required to maintain one in air. The

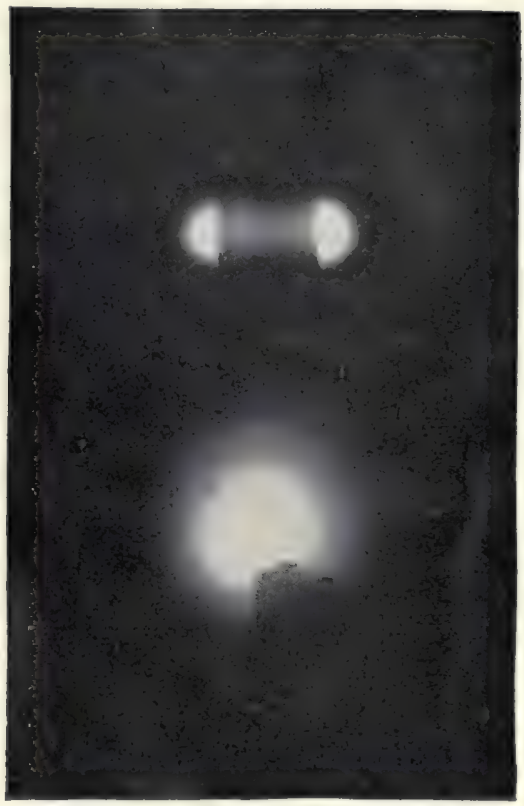

Fig. 2I.

results of some observations taken by myself are given in Fig. 22. ${ }^{1}$ Curve I represents the anode drop at different pressures, curve 2 the cathode drop, curve 3 the electric force per centimeter and curve 4 the total potential difference between the terminals. The observations were

1 Phys. Rev., 19, I20; 1904, and 20, 368; 1905. 


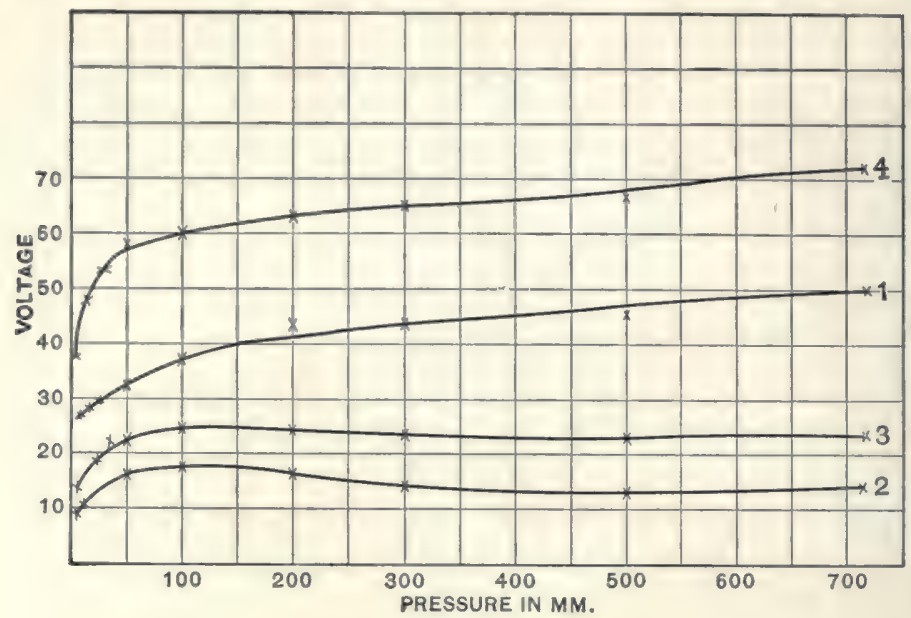

FIG. 22.

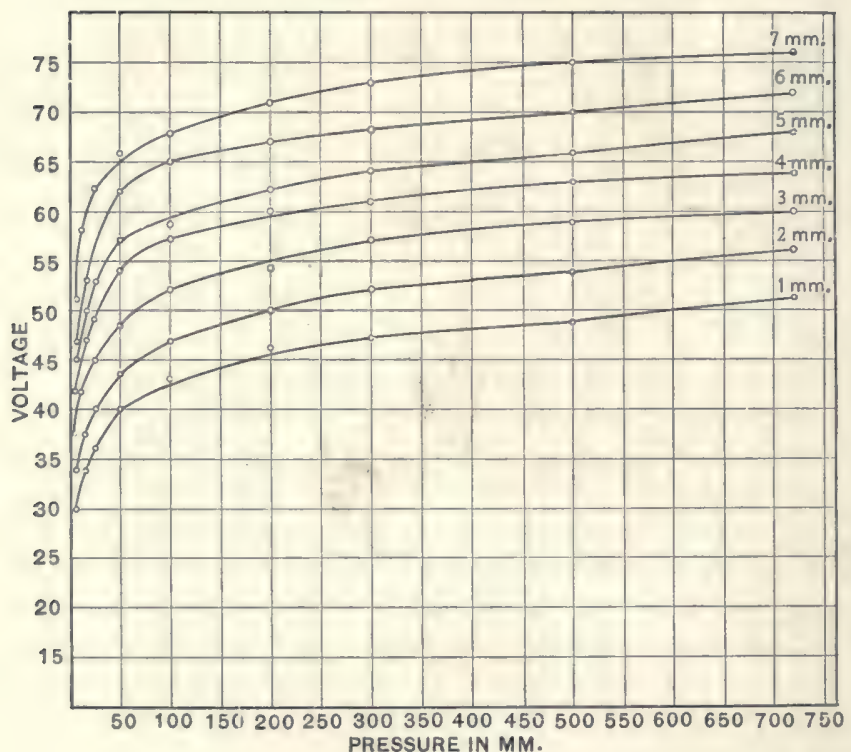

FIG. 23. 
taken with a current of Io amperes and a distance of $2 \mathrm{~mm}$. between solid carbons. ${ }^{1}$

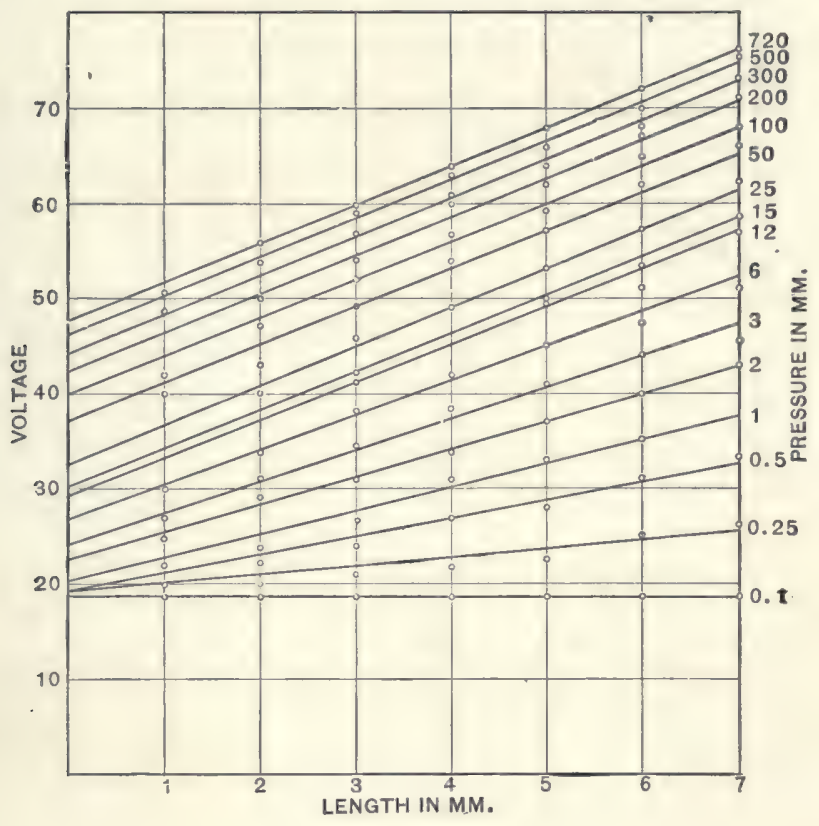

FIG. 24.

A more extended examination of the arc in a vacuum has been made by Hoerburger. ${ }^{2}$ His results are shown in Figs. 23 and 24. The first gives the relation between voltage and pressure of the gas for different lengths of the arc, the current being 6 amperes. Fig. 24 shows the relation

${ }^{1}$ As has been shown in Fig. 18, Duncan, Rowland and Todd found with longer arcs an increase in voltage when the pressure was reduced below one atmosphere. This, however, is contrary to the observations of all other experimenters.

${ }^{2}$ Dissertation Griesswald. Beib., 29, 883; 1905. 
between voltage and length of the arc for different pressures. The voltage is here shown to be a linear function of the length of the arc for all pressures. This is expressed by the formula $E=a+b l$, where $a$ and $b$ are quantities depending on the pressure of the gas. $a$ would be approx-

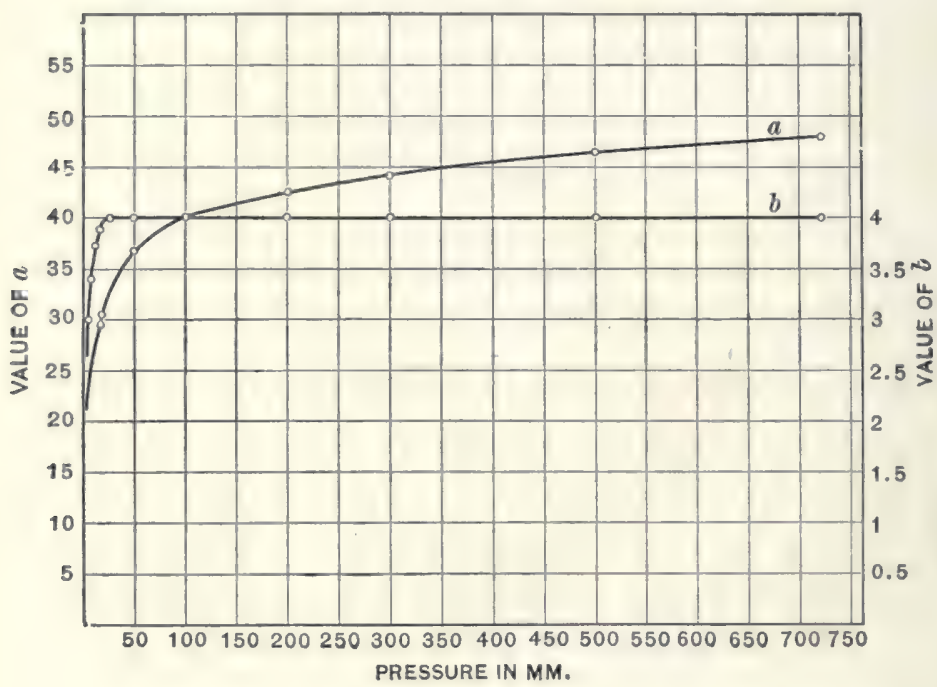

FIG. 25 .

imately the same as the sum of the anode and cathode drops, and $b$ the same as the electric force through the arc. Their values for different pressure are plotted in Fig. 25. The values for $a$ vary in the same way as those for the cathode and anode drop, as shown in Fig. 22, and the values for $b$ in the same way as the values for the electric force as found by myself. ${ }^{1}$

1 Phys. Rev., I9, I22; 1904. 
The arc in a vacuum has a lower temperature than that in air. ${ }^{1}$ This has been shown by placing a platinum wire $0.3 \mathrm{~mm}$. in diameter in the arc. At atmospheric pressures this would be melted in a fraction of a second. When the pressure of the surrounding gas is $4 \mathrm{~mm}$., the wire is not melted. With still lower pressures it gathers a deposit of carbon. ${ }^{2}$

Metal Arc in a Vacuum. - The arc between metals in a vacuum has much the same appearance as that between carbons in a vacuum, except that with the former the striations appear to be even more peculiar than with the latter. $^{3}$ The character of such an arc does not depend to any great extent on the kind of metal used for the anode. In fact, if the anode does not become so hot as to send vapor into the tube, it appears to have no effect on the discharge, so that any metal may be satisfactorily used for this electrode. Quite the opposite of this is true concerning the cathode. If the cathode is a metal which melts easily, there is no difficulty in maintaining the arc with very low pressures of the gas, ${ }^{4}$ but metals with high melting points can not be used for the cathode. ${ }^{5}$ It is, for example, impossible to maintain an arc with platinum, iron, nickel, copper or silver for the cathode when the pressure of the gas is less than I mm. and the impressed E.M.F. is no more than Ioo volts. Apparently the higher the melting point of the metal used, the more difficult it is to

${ }^{1}$ Grove, Phil. Mag., 16, 479; 1840.

2 Phys. Rev., I9, I23; I904.

${ }^{3}$ Herwig, Pogg. Ann., 149, 523; I873. Arons, Ann. d. Phys., I, 7 II; I900.

4 Stark and Kuch, Phys. ZS., 6, 438; 1905.

- Phys. Rev., 20, 369; 1905. 
maintain the arc. The following is a table of cathode drop with the metals which could be used in a vacuum.

\begin{tabular}{|l|r|}
\hline Metal. & Cathode drop. \\
\cline { 2 - 2 } & \\
Aluminum & 19 \\
Antimony & 8 \\
Zinc & 9 \\
Lead & 8 \\
Cadmium & 7 \\
Bismuth & 6 \\
Tin & 8 \\
\hline
\end{tabular}

This difficulty does not exist with oxides of metals. An arc can easily be maintained in a vacuum with oxides for the cathode. This is especially true of calcium oxide. On the other hand calcium sulphide seems to have no effect, nor the super-oxides of lead, manganese or zinc. ${ }^{1}$ An attempt was made by Arons to form an arc between terminals of Wood's metal kept liquid by being placed in a water bath near the boiling point, but in every case the tube broke almost as soon as the lamp was started. In only one case was it possible to take a reading of the current and potential difference. At that time the potential difference was between 55 and 65 volts and the current about 2 amperes.

The results with an alloy of sodium and potassium were somewhat better. Some of the lamps which contained this also broke on first starting them; others lasted sufficiently long to make observations. He found it possible to have currents from 2 to 15 amperes with a potential difference between 7 and 8 volts. Similar lamps which lasted several hours were made by Weintraub. ${ }^{2}$

$$
\begin{aligned}
& 1 \text { Stark, Phys. ZS., 5, 81; I904. } \\
& 2 \text { Phil. Mag., (6), 7, I I4; I904. }
\end{aligned}
$$


Arc in Other Gases than Air. - There have been a number of experiments performed on the arc in other gases than air with no great agreement as to results. About the only point on which there is agreement is that it is more difficult to maintain an arc in hydrogen than in air. ${ }^{1}$

In regard to the amount of potential difference needed with the arc in hydrogen the following data have been given. Arons could not maintain such an arc on a circuit of 105 volts, unless the pressure of the gas was less than one atmosphere. In some work by myself ${ }^{2}$ it was found that the voltage at the terminals of the arc was roo volts with the arc in hydrogen at a pressure of $70 \mathrm{~cm}$. With graphite terminals under the same conditions the voltage was I30 volts. Stark, Retschinsky and Schaposchnikoff give similar data for the carbon arc in hydrogen and somewhat larger values with the copper arc in hydrogen. ${ }^{3}$ Malcolm and Simon using small currents and a distance between the electrodes of $4 \mathrm{~mm}$. give the equation $E=$ $60+180 / I$.

With metals in hydrogen they found it impossible to

1 Grove, Phil. Mag., I6, 480; I840.

Matteucci, C. R., 30, 201; 1850.

Herwig, Pogg. Ann., I49, 523; I873.

Wilson and Gray, Proc. Roy. Soc., 6o, 377; 1897.

Arons, Ann. d. Phys., I, 700; 1900.

Upson, Lond. Elec., 60, 58; I908. Phil. Mag., (6), I4, I 26; I908.

S. P. Thomson states that the voltage of the arc in oxygen, nitrogen, hydrogen and other gases was the same to within one volt, the current in each case being Io amperes. (Lond. Elec., 29, 460; I892.) This result is so different from that of all other experimenters that we must consider it incorrect.

2 Phys. Rev., 20, 374; I905.

Ann. d. Phys., 18, 223; 1906. 
measure the potential difference, unless the metals were kept cool, because they melted away so rapidly. They avoided this difficulty by running a current of water through the inside of the electrode. With nickel it required from 500 to 600 volts in hydrogen while the corresponding values in air were less than roo volts.

The only other gas with which experiments have been made to any extent is nitrogen. The voltages required with this do not greatly differ from those required with the arc in air. The first data with the arc in nitrogen were given by Arons. The comparison of his results with those which had been found by von Lang for the arc between metal in air is given in the following table. The data are given for a distance between the electrodes of $1.4 \mathrm{~mm}$. and a current of 4 amperes.

\begin{tabular}{|c|c|c|c|}
\hline \multirow{2}{*}{ Metal. } & \multicolumn{2}{|c|}{ Potential difference in volts. } & \multirow{2}{*}{ Remarks. } \\
\hline & In air (v. Lang). & In N. (Arons). & \\
\hline $\begin{array}{l}\mathrm{Ag} \ldots \ldots \ldots \\
\mathrm{Zn} \ldots \ldots \\
\mathrm{Cd} \ldots \ldots \ldots \\
\mathrm{Cu} \ldots \ldots \\
\mathrm{Fe} \ldots \ldots \\
\mathrm{Pt} \ldots \ldots \\
\mathrm{A} 1 \ldots \ldots \\
\mathrm{Pb} \ldots \ldots \ldots \\
\mathrm{Mg} \ldots \ldots\end{array}$ & $\begin{array}{l}21 \\
23 \\
25 \\
25 \\
29 \\
36 \\
39 \\
\cdots \\
\ldots\end{array}$ & $\begin{array}{l}? \\
21-22 \\
21-22 \\
29-32 \\
19-22 \\
29-31 \\
26-29 \\
18 \\
21-25\end{array}$ & $\begin{array}{l}\text { With small currents. } \\
\text { With large currents. } \\
\text { With small currents. }\end{array}$ \\
\hline
\end{tabular}

Arons found it impossible to maintain an arc in nitrogen between silver terminals on a ro5-volt circuit with $6 \mathrm{ohms}$ in the circuit. On the other hand Malcolm and Simon found the voltage with silver terminals only about 20 volts higher in nitrogen than in air. 
The same experimenters give the following equations when using carbon electrodes $4 \mathrm{~mm}$. apart:

In air $E=49 \cdot 5+31 \cdot 5 / I ; \quad$ in nitrogen $E=62+30 / I$.

Grau and Russ ${ }^{1}$ examined the arc in different gases for small currents and with the distance of $5 \mathrm{~cm}$. between electrodes. The order of the gases when arranged with respect to increasing potential differences was $\mathrm{N}_{2}$, air, $\mathrm{CO}$, $\mathrm{SO}_{2}, \mathrm{O}_{2}, \mathrm{CO}_{2}$ and $\mathrm{H}_{2}$. The values for nitrogen varied from $345^{\circ}$ volts when the current was 0.05 ampere to 2075 volts when it was $0.1 \mathrm{I} 2$ ampere. For hydrogen the value of the potential difference varied from 6600 volts with a current of 0.05 ampere to 5250 volts with a current of 0.12 I ampere. This was called by them an arc, but the values of both the current and the potential difference are so different from those of the ordinary arc that one would need to know the magnitude of the cathode drop in order to decide whether this should be called an arc or a spark.

Experiments were performed by Weedon ${ }^{2}$ on the arc between water-cooled copper electrodes for the purpose of determining whether there is any law regarding the electrodes of the arc similar to Faraday's laws of electrolysis. With such electrodes in air using a current of 2 amperes for $\mathrm{I} \frac{1}{2} \mathrm{hr}$. with a potential difference of 50 volts there was a gain of weight of the anode of $0.023 \mathrm{~g}$., and of the cathode of $0.004 \mathrm{~g}$. This is not more than $\frac{1}{450}$ of the amount which would have been transferred in electrolysis and this is probably due to oxidation at high temperatures rather than to any chemical action.

1 ZS. f. Elektrochemie, 13, 345; 1907.

${ }^{2}$ Paper presented at the Electrochem. Soc., Washington, D. C., April, I904. 
With the same electrodes in hydrogen and with a form of discharge which required only 0.35 ampere and a potential difference of about 300 volts he found that the cathode lost $0.004 \mathrm{~g}$. in $\mathrm{I} \frac{1}{2} \mathrm{hr}$. and the anode gained an equal amount, but it is a question whether this was really an arc or not, so that no definite conclusion can be drawn. But the two experiments would at least show that nothing as simple as Faraday's laws will apply to the loss of material of the electrodes of the arc.

The only measurements which have been published concerning the potential in different parts of the arc in other gases than air were made by myself when working with the arc in hydrogen. It was found that with graphite terminals the larger drop was at the cathode. When the pressure of the gas was $700 \mathrm{~mm}$., the anode drop was 57 volts and the cathode drop 65 volts. On the other hand with the carbon arc in hydrogen by far the greater drop was at the anode. With $700 \mathrm{~mm}$. of pressure the anode drop was 85 volts and the cathode drop I 5 volts.

With lower pressures the behavior of the arc is much the same as it is with low pressures of air. The principal differences are found when using low pressures of hydrogen. Striations are much more prominent than they are when the residual gas is air and these appear at much higher pressures. With pressures of approximately $20 \mathrm{~mm}$. I found four distinct forms of the arc, three of these showing striations. 


\section{CHAPTER V.}

\section{The Mercury Arc.}

THE mercury arc is an arc in a vacuum having mercury for the cathode and either mercury or any other substance which does not form an amalgam with it for the anode. Tubes containing such arcs are usually called mercury vapor lamps. This lamp was first studied by Arons in $1892^{1}$ and was developed by Cooper Hewitt into a valuable commercial form in $1902 .{ }^{2}$ The commercial use has added much interest to the study of this arc and it is also by far the best form for scientific purposes. One can not, for example, begin to explain any of the phenomena of the arc without having some idea of the cause of its electrical conductivity. This requires some knowledge of the temperature of the vapor and of the electric force through this vapor, and the mercury arc offers the best chance for accurate measurements of such quantities. With this there are no complications due to chemical action and the temperature is such that detailed measurements can here be made with fine wires which do not seriously change the character of the discharge. For this reason this arc is described more fully than any other in the following pages.

For commercial use the mercury arc has several important advantages. The first of these is that the light is not

1 Wied. Ann., 47, 767; 1892.

2 Trans. Amer. Elec. Inst., 19, 59, and 20, 929; 1902. 
so concentrated as in other forms. Practically all of the light comes from the vapor, and this is usually several inches in length and about one inch in diameter. Again it has a high efficiency and does not require any attention. There is no occasion to renew electrodes nor to remove products of combustion. As long as the tube keeps out the air it is in working order and manufacturers claim a life of 5000 hours for the lamp under favorable conditions. Lastly it does not require any elaborated feeding mechanism. Nothing could be simpler than the Cooper Hewitt form of this lamp.

On the other hand it has the disadvantage of giving an unpleasant light. It is not so much that the light appears to one looking at it to be different from white light, as that it gives the wrong color to objects which it illuminates. This is due to the absence from its spectrum of some important colors. Its spectrum is a line spectrum in which only a few lines are prominent. It is lacking in red, while the green is very prominent. As a result it gives to the human face, for example, a disagreeable and lifeless appearance. The lamp is, however, of much value in certain special uses, as in a drafting room, where only the distinction between black and white is desired, or in photographic work, where the strong violet rays are of use.

The first arc between mercury terminals of which we have any record was one described by Wey. ${ }^{1}$ This, however, was an arc in the air and showed nothing of especial interest.

The first mercury arc in a vacuum was described by Arons in $1892 .^{2}$ The arc was in an inverted U-shaped glass

1 Dingler's Polytechnic. Journ., I57, 399; I860, and r59, 46; I86x.

2 Wied. Ann., 47, 767, 1892. 
tube $2 \mathrm{~cm}$. in diameter and $\mathrm{I} 2 \mathrm{~cm}$. long. Platinum terminals were sealed into the two legs and these were nearly filled with mercury. The arc was started by tipping the tube until the mercury in the two legs flowed together and then tipping it back to its original position. The following table gives the relation between current and potential difference which he found when the pressure of the residual gas was only a fraction of a millimeter.

\begin{tabular}{l|r|r|r|r|r|r|r|r|r}
\hline Current in amperes......... & II & 9 & 7 & 5.5 & 3 & 2 & 1.4 & 0.8 & 0.5 \\
Potential dif., in volts.... & 17.5 & 17 & 16.5 & 16 & 15.3 & 14 & 20 & 28 & 40 \\
\hline
\end{tabular}

With currents greater than I.4 amperes the tube was filled with light. At the cathode there was a spot of intense light which was in constant motion. With small currents stratifications occasionally appeared.

The presence of small amounts of air or hydrogen did not greatly affect the relation between the current and the potential difference. For example, when the pressure of the remaining gas was $5 \mathrm{~mm}$., there was a current of 8.5 amperes with a potential difference of 18 volts, which is but little different from that given above for the same current. In that case, however, the light did not fill all of the tube, but appeared as a band in the central part, and the glass became heated very quickly.

A few years later Arons found that the mercury vapor loses its conductivity very quickly after the arc has been extinguished and that it is not possible to have an alternating-current arc with such a lamp. ${ }^{1}$

The commercial form of this arc as developed by Cooper Hewitt is shown in Fig. 26. In this lamp both terminals are mercury. It is started by tipping the tube so that the

1 Wied. Ann., 57, 192; 1896 , and 58, 73; 1896. 
mercury in the lower bulb flows along and makes contact with that in the upper one. The tube is then tipped back into the position shown in the figure, which breaks the contact and starts the arc.

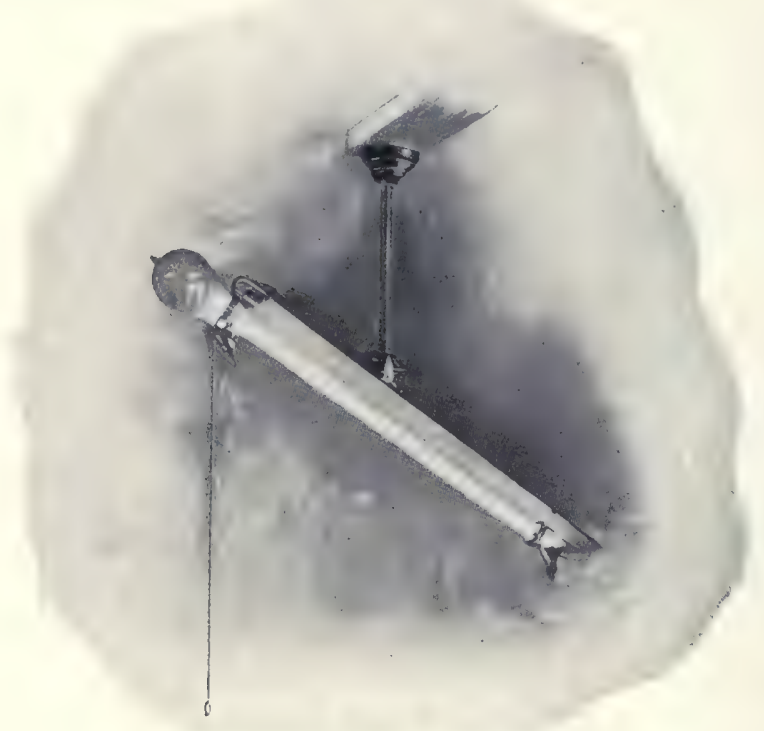

FIG. 26.

The mercury lamp made by the General Electric Company is shown in Fig. 27. Here the anode is a cylinder of carbon and the arc is started by a fine carbon filament running through the lamp and dipping into an iron cup which floats on the mercury at the bottom of the tube. As soon as the current is started this cup is drawn down by a solenoid about the outside of the tube, through which the current flows. As it is drawn down, an arc is started 
at the bottom of the filament, which quickly extends the whole length of the tube, since the resistance of the carbon filament is much greater than that of the mercury vapor after the arc is started.

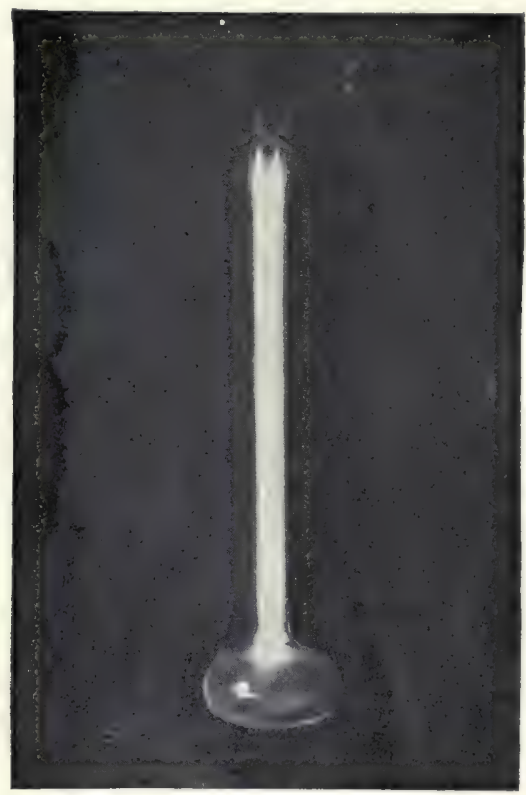

FiG. 27 .

Temperature of the Mercury Arc. - As has been stated the mercury arc affords better opportunities than other arcs for the accurate measurements of those quantities which are important in forming a theory of the discharge. While this is true, there are still several of these about which we are more or less ignorant. Either the different observers have recorded different results, or else some valid criticism may be made on the methods used. 
One of these quantities is the temperature of the mercury arc. Nothing would seem simpler than to insert a thermometer or thermojunction in the mercury vapor and observe its temperature. There are, indeed, several who have made such measurements. Thus Arons measured this temperature by means of a mercury thermometer and found that it varied from $285^{\circ} \mathrm{C}$. to $5^{1} 5^{\circ} \mathrm{C}$. Wills ${ }^{1}$ measured it with a thermojunction and found values varying from $83^{\circ} \mathrm{C}$. to $297^{\circ} \mathrm{C}$., depending on the length of time that the arc had been running and on the density of the mercury vapor. Knipp also measured this temperature with a thermojunction and showed that the temperature increases rapidly as the pressure of the vapor increases. ${ }^{2}$ However, both the thermometer and the thermojunction give temperatures which are too low. Both radiate some heat and conduct some away and must be cooler than the vapor in order to receive heat from it. Arons, therefore, made a further test by placing a fine platinum wire in the vapor of the arc. He found that this was quickly melted. There is, however, the possibility that the platinum wire first formed an amalgam with the mercury and that this caused the wire to melt at a comparatively low temperature. I have, therefore, repeated the experiment using a glass fiber and find that this also can be melted in the mercury arc, but even here we are not on sure ground, for the glass may receive its heat from the moving electrons and not from the molecules of the vapor.

Arons endeavored to compute the temperature of the mercury vapor. We know approximately the amount of gas in the tube, its specific heat and the rate at which

1 Phys. Rev., 19, 65; 1904.

2 Phys. Rev., 24, 446; 1907, and 31, 97; 1910. 
energy is given to it. If we had any definite knowledge concerning the relation between the temperature of a luminous vapor and the rate at which it radiates heat, it would be a simple matter to make this computation, but while we know something about the rate at which a nonluminous vapor radiates heat, there is a small portion of the gas which is luminous and this radiates energy at a very much greater rate. Arons did indeed make some assumption concerning this and arrived at the conclusion that the temperature of the vapor must be $4000^{\circ} \mathrm{C}$. or $5000^{\circ} \mathrm{C}$. This certainly is much too high, so that it would appear that theoretical considerations help us but little with this problem.

For the same reason we can not accept the measurements of this temperature made by Fery, ${ }^{1}$ who compared the intensity of the light in different parts of the spectrum of this arc with corresponding parts of the spectrum of the carbon arc and concluded that the temperature of the mercury vapor was $3500^{\circ} \mathrm{C}$. This temperature may possibly be the correct temperature for the luminous part of the vapor, but can hardly be correct for the non-luminous part.

It indeed seems more probable that the measurements made with a thermojunction are not far from the correct value, at least for pressures of several millimeters. However, as the density of the vapor becomes less the error will become greater and there is no means known for determining the exact amount of the error.

Characteristic Curves for the Mercury Arc. - A knowledge of the relation between the current and the voltage is also important in forming a theory of the arc, and it is also

${ }^{1}$ Soc. Franc. Phys. Bull., No. 3, 305; 1907. 
difficult to determine this relation. It is not possible to change the current with the arc in a tube without changing the pressure of the vapor through which the current is passing, and a change in the pressure of the vapor will in itself cause a change in the voltage required irrespective of any effect which the current may have.

Moreover, the amount of change in the vapor pressure depends on several factors. If, for example, the tube containing the arc is connected to a pump the vapor produced by the arc passes into other parts of the apparatus where it is rapidly condensed and the pressure in the arc does not become large. If the lamp is not connected to other apparatus, the pressure amounts to several millimeters, and with quartz tubes where large currents are used the pressure may become even more than one atmosphere.

Again the pressure depends to a great extent on such things as the size of the tube, the condensing chambers and the rate at which heat is taken from the tube. The hotter the tube becomes, the more mercury will be vaporized and the greater the pressure of the vapor. There will, therefore, be a great difference between a tube which is placed in water kept at a constant temperature and a tube surrounded by air. Even a difference in the thickness of the walls of a tube may easily affect the rate at which heat is radiated and consequently the pressure of the vapor, and the size of the tube may not only affect the pressure by giving more room for the vapor, but may affect it indirectly by giving more chance for the heat to escape.

Moreover it is not easy to make accurate determinations of this pressure when it is low. One can not use any arrangement similar to a McLeod gauge, since the vapor condenses to a liquid as soon as it is taken from the lumi- 
nous column. With pressures greater than one millimeter fairly accurate measurements can be made by reading the difference in level between the two arms of a manometer, but with pressures less than this such readings have serious errors and these lower pressures are important ones in forming a theory of the arc.

Wills used the following indirect method of finding the vapor pressure. The temperature was first determined by the method already described and from this, by using tables which give the relation between the pressure of mercury vapor and its temperature, the pressure was computed. This assumed that the thermojunction gave the correct value of the temperature and also that the pressure in the arc is the same as it would be, if the vapor and the liquid in contact with it were at the same temperature, which is the condition present when measurements are made on vapor pressure. The first of these assumptions has already been criticized and the second is certainly incorrect. There can be no question but that the vapor through which the current is passing is very much hotter than the liquid, which is only a little warmer than the surrounding air.

However, some idea of the relation between voltage and current can be had from the curve given by Cooper Hewitt, ${ }^{1}$ which is shown in Fig. 28. The circles on the continuous curve indicate the final voltages between the terminals of a mercury lamp with different currents. The crosses indicate readings which were taken immediately after the current had been increased and before the pressure of the gas had changed to any great extent. Thus the cross at $a$ indicates the reading when the current had suddenly been increased ${ }^{1}$ Elec. World, 37, 679; 190r. 
from 4.8 amperes to 6.1 amperes. As the pressure of the gas increased the current gradually decreased and the voltage increased, until finally the point $b$ was reached.

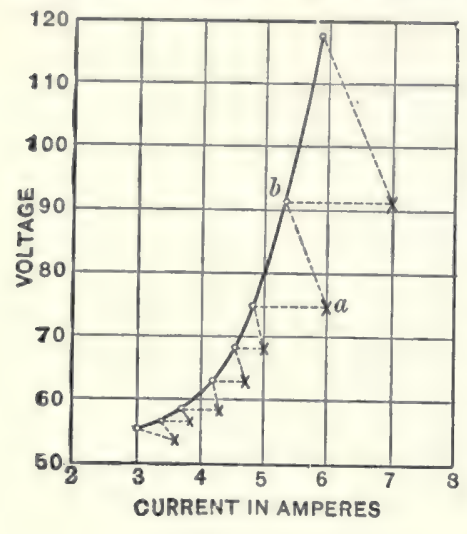

FIG. 28.

The dotted line in this figure indicates that the voltage is very nearly constant when there is no change in the density of the vapor, even when large changes are made in the current. This probably comes very near to the true relation between the voltage of the mercury arc and the current with constant pressure.

The very great increase in the voltage required with increased vapor pressure of the mercury arc in quartz tubes will be shown in a following paragraph.

The Cathode Drop of the Mercury Arc. - In a similar way we find it difficult to determine just what the drop in potential in different parts of the arc really is. Thus Arons found the cathode drop to be 5.4 volts. Wills found it to be approximately 5 volts and Pollak gives it as slightly more than this. Knipp ${ }^{1}$ measured the drop in potential between a platinum exploring wire and a mercury cathode when the current was 3 amperes and found the cathode drop to vary from 6 volts when the pressure of the gas in the pump connected to the lamp was $0.083 \mathrm{~mm}$. to 6.8 volts when the pressure was $1.9 \mathrm{~mm}$.

Stark, Retschinsky and Schaposchnikoff ${ }^{2}$ used mercury
1 Phys. Rev., 31, 106; I9Io.
2 Ann. d. Phys., 18, 243; 1905. 
for the exploring electrode, so that no potential difference was introduced by using a different metal from that of the cathode. They found the cathode drop to be $5.27 \pm 0.09$ volts and stated that it is independent of the current.

The Anode Drop of the Mercury Arc. - The anode drop has two distinct values; the first when the anode is in the direct path of the cathode rays, that is, when it is not covered by any glow, or "anode layer" as it has been called by Stark; the second value occurs when there is this glow. In the first case Stark, Retschinsky and Schaposchnikoff, using an iron anode, found the anode drop to vary from 3.14 volts with a current of 3.6 amperes to 3.65 volts with one of 9.3 amperes.

In the second case the anode drop would appear to be in the neighborhood of 5 volts. Arons found it to be 7.4 volts. Wills found it to be 6.5 volts with a current of 1.25 amperes, decreasing to 4.6 volts with one of 3.25 amperes. These measurements were made with an exploring wire $\mathrm{I} \mathrm{cm}$. from an iron anode. Pollak ${ }^{1}$ found the value $6 \mathrm{~mm}$. from the anode to be 5.7 volts when the current was 6.7 amperes. Knipp measured the drop in potential between a platinum anode and a platinum exploring wire when the current was 3 amperes and found the anode drop to vary from 3.6 volts when the pressure of the gas in the apparatus connected with the tube was $0.083 \mathrm{~mm}$. to 5.4 volts when the pressure was $3 \mathrm{~mm} .^{2}$

The Electric Force in the Mercury Arc. - The electric force required to send a current through the vapor of a mercury arc is surprisingly small. Ordinarily the resistance of a column of gas is very high, even when there is a

1 Ann. d. Phys., 19, 217; 1906.

2 See also Phys. Rev., 30, 319; I9ro. 
large amount of ionization. Even with a carbon arc in air the resistance of the gaseous part with a current of six amperes is approximately $5.5 \mathrm{ohms}$ per centimeter. In an experiment by Knipp when the tube containing the mercury arc was $22.4 \mathrm{~cm}$. long and $3.4 \mathrm{~cm}$. in diameter and the current was 3 amperes, the resistance was as low as O.I3 ohm per centimeter. With larger currents it is even less.

The electric force in the mercury arc depends on the pressure of the vapor, the diameter of the tube, the amount of current and the temperature. As the pressure is increased there is a decided increase in the electric force required. When working with the tube mentioned above Knipp found the electric force to vary from 0.39 volt per centimeter, when the pressure of the gas in the pump connected with the tube was $0.083 \mathrm{~mm}$. to 0.78 volt per centimeter when the pressure was $3.0 \mathrm{~mm}$. The work of Wills is quite in agreement with these data.

Wills found that the larger the tube the smaller the electric force required. For example, the electric force with a current of 2 amperes and a pressure of I mm. varied from 0.98 volt per centimeter when the diameter of the tube was I $\mathrm{cm}$. to 0.37 volt per centimeter when the diameter was $8 \mathrm{~cm}$. Arons also found the force to be greater with smaller tubes.

The electric force decreases as the current is increased, if the pressure of the mercury vapor remains constant. In some data which I hope soon to publish, it is shown that doubling the current causes the electric force to decrease about io per cent.

Pollak ${ }^{1}$ measured the potential through an arc when the 1 Ann. d. Phys., 19, 239; 1906. 
current was 6.7 amperes and the pressure of the vapor was $1.33 \mathrm{~mm}$. This is shown in Fig. 29. The total potential difference was 22.5 volts. It is of interest to note that there is a point near the anode? where the electric force is in the opposite direction to that in which the current is flowing. This is no doubt due to an accu-

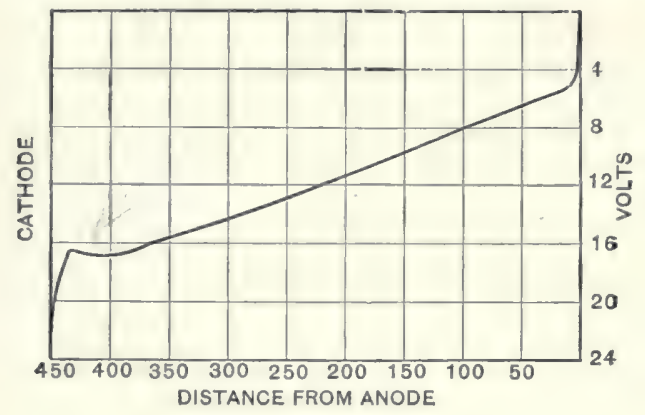

FIG. 29.

mulation, in this neighborhood, of ions whose electrostatic effect is sufficient to reverse the original direction of the electric force. This accumulation is due to the momentum of the ions which carries them for a short distance against the electric force. ${ }^{1}$

The vapor of the mercury arc remains highly conducting and quite luminous even after it has passed from the arc into a condensing chamber. Stark ${ }^{2}$ found that if this vapor is passed through a magnetic field, an E.M.F. is induced, which is perpendicular to the field and to the direction of the motion of the vapor. The magnitude of this E.M.F. indicates a velocity of the vapor of approximately 280 meters per second.

1 Phil. Mag., (6), I8, 442; I909.

2 Phys. ZS., 4, 440; 1903. 
In some work by myself ${ }^{1}$ it was shown that the front of this luminous region where the mercury is condensing to the liquid form has the greatest conductivity and the greatest luminosity. It was also shown that this conductivity is not due to any rays sent out from the arc itself.

Mercury Arc in Quartz Tubes. - The light efficiency of mercury vapor lamps is higher with large currents, but tubes made of ordinary glass will melt when such currents are used. To avoid this difficulty lamps are now being made of fused quartz. These lamps are among the very best as far as watts per candle power are concerned. They also give off a great amount of ultra-violet light, which is advantageous for photographic work, and because of these rays they can be used for sterilizing water.

The light from such lamps appears to the eye whiter than that from the common mercury vapor lamps and yet it is lacking in certain important parts of the spectrum and has the added disadvantage that the ultra-violet rays are very injurious to the eyes. However, these rays may be screened off by common glass, there being sufficient protection, if the quartz lamp is placed inside a glass globe. It is also claimed that the rays are absorbed by a few feet of air, so that lamps placed fifteen or twenty feet above a sidewalk would not be injurious to passers-by, even if the glass globe should be broken.

The relation between current, potential difference and the pressure of the mercury vapor in such lamps has been examined by Kuch and Retschinsky. ${ }^{2}$ As the tube was allowed to become hot the vapor pressure rose and a larger potential difference was necessary in order to maintain

1 Phys. Rev., 22, 221; 1906.

2 Ann. d. Phys., 20, 563; Igo6. 
the arc, although it did not increase as rapidly as the pressure. The following is an abridgement of the table given by them.

\begin{tabular}{|c|c|r|}
\hline $\begin{array}{c}\text { Potential } \\
\text { difference. }\end{array}$ & Current. & Pressure in cm. \\
\cline { 1 - 1 } 36 & 2.78 & 0.2 \\
67 & 4.20 & 9.0 \\
114 & 4.50 & 29.5 \\
154 & 4.80 & 58.7 \\
202 & 4.80 & 100.5 \\
237 & 4.50 & 138.0 \\
249 & 4.40 & 150.0 \\
\hline
\end{tabular}

Kuch and Retschinsky also examined the temperature in quartz lamps using a thermojunction. ${ }^{1}$ They found that at the middle of the tube the temperature became as high as $1710^{\circ} \mathrm{C}$., which was the highest that their thermojunction would register. The temperature at the sides was at all times lower than that in the middle.

Velocity of the Ions in the Mercury Arc. - The velocity of the negative ions in the mercury arc is much greater than that of the positive ones. ${ }^{2}$ This has been shown by placing two secondary electrodes in the conducting vapor of this arc and passing a current between them. On examining the potential between these electrodes, it is found that the drop in potential near the positive is many times greater than that near the negative. As will be shown in the discussion of the theory of electrical discharge, this shows that the negative ions have the greater velocity.

The same thing is shown by the work of Schenkel ${ }^{3}$ on the

1 Ann. d. Phys., 22, 595; 1907.

2 Stark, Retschinsky and Schaposchnikoff, Ann. d. Phys., 18, 230; 1905.

${ }^{3}$ Mitt. d. Phys. Ges. Zurich, p. 13, 1906. 
Hall effect in the mercury arc. He states that $\left(k_{2}-k_{1}\right) p$ is $1.42 \times 10^{7} \mathrm{~cm}$. per second per volt, where $k_{1}$ is the velocity of the positive ions, $k_{2}$ that of the negative ions and $p$ the pressure of the gas measured in millimeters.

Modifications of the Arc to Produce White Light. - The absence of some of the colors in the mercury arc is an objection to its use and many attempts have been made to supply the missing colors by introducing other substances with the mercury. For this purpose amalgams of mercury have been tried, but none of them have proven altogether successful. With the currents and temperatures commonly used the substance amalgamated with the mercury takes little or no part in the passage of the current. This probably is due to the mercury alone being vaporized.

Several amalgams were tried by Arons who found that the potential difference was even less with sodium and potassium amalgams than with pure mercury. With a potassium amalgam only io volts were needed to maintain a current varying from 3 to 6 amperes. But unfortunately these lamps give less light than the common mercury vapor lamp.

When the tube was cooled by being placed in the water bath no potassium lines appeared in the spectrum of the arc, even though twenty amperes of current were used. When the lamp was not cooled the potassium lines were apparent with five or more amperes.

With silver amalgams the potential difference was somewhat larger than with pure mercury. The spectrum of the silver appeared only at the cathode and then only one line, except when the lamp was run for a long time. In that case this line disappeared and two others of the silver spectrum appeared in its place. There was excessive heat- 
ing when a zinc amalgam was placed in the tube or pieces of aluminum were allowed to float on the mercury. With both tin and aluminum amalgams the potential difference. was much higherr than with pure mercury.

More successful results were attained by Gehrke and Baeyer ${ }^{1}$ who found that an amalgam of 100 parts, by weight, of zinc and 30 parts of mercury gives a much better light than mercury alone. Still better results have been secured when using amalgams in quartz tubes, since these tubes can stand a higher temperature and are not acted on chemically. Arons ${ }^{2}$ recommends the following for use in quartz tubes: mercury 59 per cent, lead 20 per cent, bismuth 20 per cent, zinc 0.5 per cent and cadmium 0.5 per cent.

Quite a different method of supplying the deficient light has been suggested by Cooper Hewitt. ${ }^{3}$ This is to use a reflector on whose surface are crystals which emit red light when illuminated by ultra-violet rays. No data have as yet been published concerning the amount of red light which is thus obtained and it does not seem to have met with any commercial success.

Another device and one which promises excellent results is a lamp described by Urbain, Scal and Feige. ${ }^{4}$ In this the anode is tungsten and the density of the current is such that this is raised to a very high temperature and gives out the greater part of the light. This must be in a vacuum or in an inert gas, since a trace of oxygen is sufficient to blacken the surrounding globe. The principal use of the mercury is to start the arc, it being difficult to start an arc

\footnotetext{
1 Elektrot. ZS., 27, 383; 1906.

2 Lond. Elec., 62, 387 ; $x 908$.

${ }^{3}$ Elec. World, 56, r343; r9ro.

4 L'Industrie Elec., March Io, rorr.
} 
in an air-tight globe by other means. The length of the column of luminous mercury vapor is only a few millimeters. The color is bluish white, very similar to sunlight, and it is said to require but 0.45 watt per candle. It operates on a potential difference as low as $\mathrm{I} 2$ volts. The voltage and candle power may be made greater by having an inert gas in the tube.

The lack of red in the mercury arc is occasionally remedied by combining the arc with a tungsten incandescent lamp. It is usually necessary to have some resistance in series with the arc. The tungsten lamp furnishes this resistance and at the same time serves the useful purpose of giving the lacking colors, thus making an efficient combination. 


\section{CHAPTER VI.}

\section{Alternating-Current Arcs.}

THE preceding discussion has been on arcs produced by direct currents. These are more efficient than alternatingcurrent arcs and would probably be used in every case, if it were always convenient to have direct currents. Since it is not, alternating-current arcs have a somewhat limited use. The differences between the two kinds of arcs are in general such as might well be expected. There is, however, very great difficulty in maintaining alternating-current arcs between metals, as will be shown later.

Alternating-current Arc between Carbon Terminals. With carbon terminals the appearance of the alternatingcurrent arc is much the same as that of the direct-current arc, except that the two electrodes appear alike in the former case. This, of course, is due to the rapid change of polarity of the terminals. However, with the frequencies commonly used, the light appears to the eye to be continuous, but it is easy to show with a revolving mirror that the luminosity of the region between the carbons fluctuates greatly, the vapor being comparatively non-luminous for a short period between each alternation. ${ }^{1}$ The carbons remain luminous, although they also pass through quite appreciable changes with each alternation.

This arc is accompanied by a humming sound corresponding to the frequency of alternations. In general, this

${ }^{1}$ Lum. Elec., 42, 55I and 618; I89I. 
humming is so loud as to make the arc disagreeable when in a closed room. It is more noticeable with solid than with cored carbons.

The possibility of having an alternating-current arc is due to the fact that the region between the carbons remains conducting for an appreciable length of time after the current has ceased to flow. This fact was known as early as 1867 when Le Roux ${ }^{1}$ found that he could start an arc $0.04 \mathrm{sec}$. after the removal of the E.M.F.

Characteristic Curves. - Characteristic curves for alternating-current arcs show the same general shape as those for direct-current arcs. With both the voltage becomes greater as the length of the arc is increased and less as the current is made greater. However, the mean voltage of the alternating-current arc is less than that for the directcurrent arc of the same length and current. Heubach ${ }^{2}$ gives the equation $E=20.4+\mathrm{I} .8 l$ for the alternatingcurrent arc between cored carbons, when the current is 6.5 amperes and $E=19.78+2.2 l$, when the current is 4.4 amperes. As usual, $E$ is measured in volts and $l$ in millimeters. This gives, for example, a voltage of 29.4 volts for an arc $5 \mathrm{~mm}$. long having a current of 6.5 amperes, as compared with 55 volts, the value given by Mrs. Ayrton for a direct-current arc having the same current and length between a cored anode and a solid cathode. The fact that both of the carbons were cored in the alternating-current arc no doubt explains part of the difference, but only a small part. In some observations made by myself in using cored carbons for both alternating- and direct-current arcs it was found that the voltage for the former was from

1 C. R., 65, II49; 1867 .

2 Elektrot. ZS., 13, 460; 1892. 
I 5 to 20 volts lower than that for the latter, the current and length of arcs being the same in both cases.

The characteristic curves for the flaming arc with alternating current are nearly identical with those for the same arc with direct current. One curve taken by myself for a flaming arc Io $\mathrm{mm}$. long with alternating current was identical throughout the greater part of its length with that taken with the same carbons and length where a direct current was used, as shown in Fig. I6.

Current and Potential Difference at Different Phases. As far as I have been able to learn the first published work on the alternating-current arc was by Joubert, in $1880,{ }^{1}$ who showed the values of the potential difference and the current at different phases of the alternations. His method was the one which is well known in the work on alternating currents, where a measuring instrument is connected to the circuit for a brief length of time during each alternation, the connection being always made at the same phase of the alternation. He found the curve for the current to be nearly a sine curve, while that for the potential difference was greatly deformed. This observation was confirmed by Tobey and Walbridge. ${ }^{2}$ The form of these curves has been found to vary with the kind of dynamo used, the character of the carbon and the length of the arc. ${ }^{3}$

The preceding method gave the average value of some

1 C. R., 9I, I6r; I880.

2 Amer. Inst. Elec. Eng., 7, 367; I89o.

3 Rossler and Wedding, Elektrot. ZS., I5, 3I5; I894. Fleming and Petavel, Phil. Mag., (5), 4I, 3I5; I896.

Frith, Phil. Mag., (5), 4I, 507; 1896.

C. S. Smith, Lond. Elec., 39, 855; 1897.

Beckit Burnie, Lond. Elec., 39, 849; 1897.

Eichberg and Kallir, Wien. Sitzungbr., I07, 2A, 658; I898. 
particular phase of the current or potential difference during many periods. The oscillograph devised by Blondel $^{1}$ gives a photograph of these curves during the whole of some one period. This instrument is in principle a galvanometer, the period of whose needle is very short, so that the mirror is able to follow closely each fluctuation of the current. A spot of light may be reflected from this

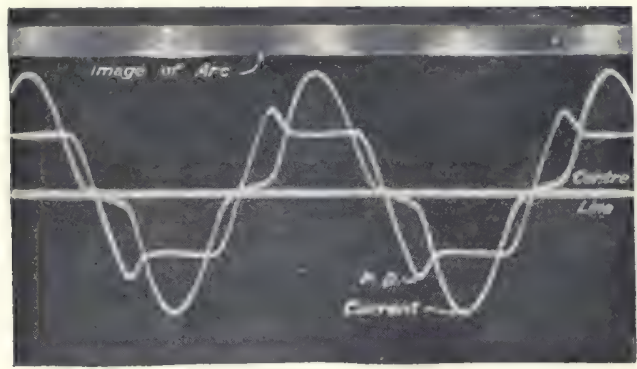

FIG. 30.

mirror to a rapidly moving photographic film which records the changes in the current. One such device may be put in series with the arc and another in shunt with it, so that records of the current and voltage may be made on the same film and compared at each phase of the alternation, as in Fig. $300^{2}$

It will be seen from this that the current does not at first increase rapidly, while the voltage reaches its highest value in a small fraction of a cycle. This is due

1 C. R., 127, rorg; 1898 .

2 An improvement in this instrument was made by Duddell and Marchant (Journ. Ins. Elec. Eng., 28, I; I899) and a very complete set of curves with different kinds of earbons, period of alternation, currents and lengths of arc were given by them. 
to the fact that a potential difference of several volts is necessary in order to produce any current at all through the arc.

As the current increases the voltage decreases slightly, since it requires less voltage to maintain a large current than a small one. The amount of the current during this part of the cycle is determined chiefly by the resistance and inductance in the other parts of the circuit. Near the end of the phase the current rapidly approaches zero, since the voltage has then become less than that needed to maintain an arc.

There is given in Fig. $3 \mathrm{r}$ a curve taken with an oscillograph where the voltage curve $a a^{\prime}$ is given in a negative direction in order that it may not be confused with the current curve $b b^{\prime}$. 'These curves were taken by Simon $^{1}$ with an

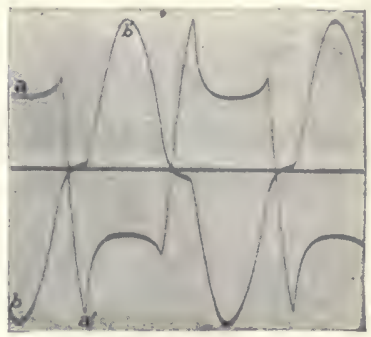

FIG. 3I. arc between solid carbons $5 \mathrm{~mm}$. in diameter placed horizontally above a Bunsen burner, so that the arc was in the flame of the burner. The arc was $2.5 \mathrm{~mm}$. in length and the current was 2.3 amperes with a frequency of 50 per second.

The flaming arcs behave with alternating currents much the same as the arc between cored carbons, although the curves of the flaming arc taken with an oscillograph were more similar to those of the solid than to those of the cored carbon arcs. $^{2}$ Fig. 32 gives such curves when there was non-inductive resistance in series with the arc.

1 Phys. ZS., 6, 305; 1905.

2 Blondel, Trans. Intern. Elec. Cong., 2, 744; 1904. 


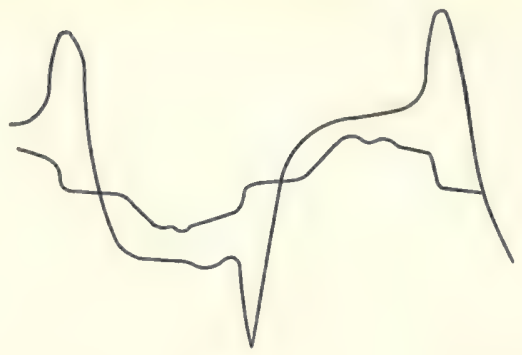

FIG. 32.

Dynamical Characteristic Curves. - Mention has already been made of what were called "dynamical" characteristic curves. Such curves show the relation between the potential difference and the current for arcs which are

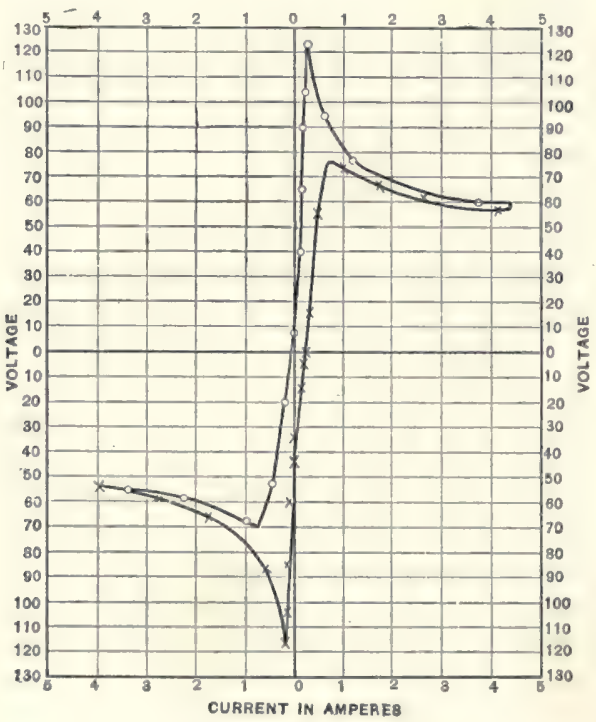

FIg. 33. 
rapidly changing. Thus, if the different values of the current as shown in Fig. $3 \mathrm{I}$ are taken as abscissæ and the corresponding values of the potential difference as ordinates, one would have the curve given in Fig. 33, which is a dynamical characteristic curve.

This gives in a different way some of the things shown by Fig. 30 and also calls attention to the fact that the relation between potential difference and current is not the same when the current is increasing as when it is decreasing. Thus the part of the curve at the left indicates the relation existing when the current is changing from the greatest negative current to the maximum positive. The curve on the right indicates the relation when the current is changing from positive to negative.

When the current is increasing, the carbons are not so hot as they are when it has been large and is becoming small. With the cooler electrodes the potential difference is greater than when the electrodes are hotter, although the current is the same. This peculiarity of the curves is called by Simon "arc-light hysteresis."

These curves are quite different from those shown in Figs. 3-7. There the potential difference approached infinity as the current approached zero. Here the two approach zero together. The reason is that the part of the curve near the origin does not strictly

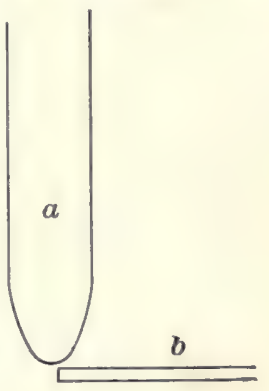
refer to an arc. It refers rather to the simpler form of 
discharge between two hot bodies where there is no ionization by impact at the surface of the electrode, as, for example, the discharge from a hot piece of iron or platinum.

With this simpler form of discharge the current will become smaller as the voltage becomes smaller. The two do not necessarily approach zero at the same time, there may be a small E.M.F. due to the difference in twoperature between the two bodies and since it takes an appreciable time for the ions to move across the gap. But the characteristic curve for such a discharge would at least go near the origin, as do the curves in Fig. $33 .{ }^{1}$

Fall of Potential in Alternating-current Arc. - The fall of potential in different parts of the alternating-current arc
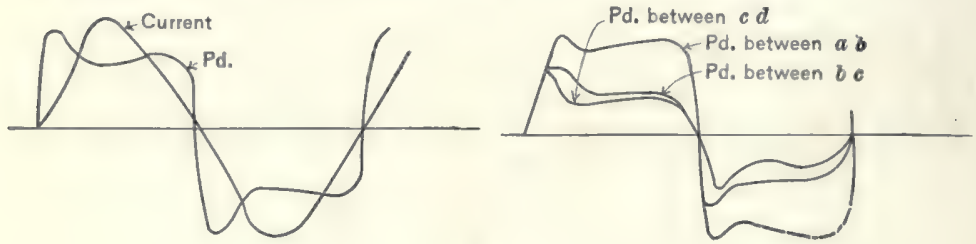

FIG. 35.

was examined by Duddell and Marchant. Their results are shown in Fig. 35. Fig. 34 shows the position of the exploring carbons. The left hand part of Fig. 35 shows the curves of current and total potential difference, while the part on the right shows the potential difference in different parts of the arc. All the curves of potential difference show the same general shape. The carbons used were "Apostle" carbons $13 \mathrm{~mm}$. in diameter. The arc was $6 \mathrm{~mm}$. long

1 A very complete study of dynamic characteristic curves with the arc in various gases and in air at reduced pressures has been given by Gertrude Lange (Ann. d. Phys., 32, 589; 1910). 
and there were 14.8 amperes and 97 alternations per second.

Phase Difference in the Alternating-current Arc. - As was shown in Fig. 30 the current lags somewhat behind the potential difference. As a result the power given to the arc is not equal to the product of the current as $\eta$ fured by an alternating-current ammeter times the volta as measured by a voltmeter. In this respect the arc behaves as a resistance having self-induction. The phenomenon, however, is not due to self-induction, but to the fact that the conductivity between the carbons is not so good when the arc has momentarily been extinguished and the gas and electrodes have become cooled as when the current has been flowing.

The existence of this apparent phase difference was pointed out by Heubach ${ }^{1}$ who found it to exist for solid carbons but not for cored carbons. In the following table the value for $c$, the power factor, is given for different carbons and with different alternations per second. ${ }^{2}$

\begin{tabular}{|c|c|c|c|}
\hline \multicolumn{2}{|c|}{ Carbons. } & \multirow{2}{*}{$\begin{array}{l}\text { Alternations } \\
\text { per sec. }\end{array}$} & \multirow{2}{*}{$c}$. \\
\hline Upper. & Lower. & & \\
\hline \multirow{4}{*}{ Solid } & \multirow{4}{*}{ Solid } & 127 & 0.870 \\
\hline & & 97 & 0.865 \\
\hline & & 70 & 0.805 \\
\hline & & $\begin{array}{r}57 \\
127\end{array}$ & $\begin{array}{l}0.750 \\
0.975\end{array}$ \\
\hline \multirow{2}{*}{ Solid } & \multirow{2}{*}{ Cored } & 97 & 0.965 \\
\hline & & 70 & 0.935 \\
\hline \multirow{4}{*}{ Cored } & \multirow{4}{*}{ Cored } & $\begin{array}{r}46 \\
127\end{array}$ & $\begin{array}{l}0.915 \\
0.985\end{array}$ \\
\hline & & 97 & 0.980 \\
\hline & & 70 & 0.970 \\
\hline & & & \\
\hline
\end{tabular}

1 Elecktrot. ZS., 13, 460; I892.

2 Duddell and Marchant, Journ. Inst. Elec. Eng., 28, 86; 1899. 


\section{Alternating-current Arc between Metals. - Mention} was made by Jamin and Maneuvier ${ }^{1}$ in 1882 of alternatingcurrent arcs having a metal for one terminal and carbon for the other, but, curiously, they make no mention of what will occur when both terminals are metals. The difficulty of maintaining such an arc was no doubt well known, but the first observation to which I have been able to find a reference was made by Wurts in I892. ${ }^{2}$ He found that he could not maintain such arcs between zinc, cadmium, bismuth or antimony, even with large voltages. These he called "non-arcing" metals. They are still often called this, although the work of other experimenters would indicate that they are no more non-arcing thar many other metals.

Arons ${ }^{3}$ using an E.M.F. of 360 volts was unable to maintain such an arc between mercury, aluminum, copper, iron, platinum, brass or nickelin. He believed that it would be necessary to have an E.M.F. sufficiently high to start the discharge each time with a spark.

Similar observations were made by Granqvist ${ }^{4}$ who believed that the difficulty was due to the fact that the heat is rapidly conducted away by the metal. Steinmet ${ }^{5}$ states that it is necessary to have 500 volts in order to maintain an alternating-current arc between magnetite terminals and then it is very unsteady and the current is partially rectified. In some experiments performed by myself it was found that this form of arc could not be

1 C. R., 94, 1615; 1882.

2 Amer. Inst. Elec. Eng., 9, ro2; 1892.

s Wied. Ann., 57, 195; 1896.

4 Upsala Rom. Soc., p. I; r903. Phys. ZS., 4, 537; 1903. Science Abs., 7, 40; 1904, and 10, 344; 1907.

5 Trans. Intern. Elec. Cong., St. Louis, 2, 7 19; I904. 
maintained between iron and copper terminals either in air or in a vacuum with an E.M.F. of 220 volts. $^{1}$ With graphite it was very difficult to maintain an arc with such a voltage.

There appears to be no further work with such arcs where the current was comparable with that of the common arc. There have, however, been two investigations where very small currents and high E.M.F.'s were used, but it is difficult to decide whether these should be considered as arcs or as sparks.

The first of these investigations was made by Guye and Monash. ${ }^{2}$ They concluded from their experiments that the difficulty of obtaining the alternating-current arc between metals increases as the atomic weight of the metal becomes greater. In the following table there are given the potential differences with the metals investigated by them when the current was 0.04 ampere, the length of the arc $5 \mathrm{~mm}$., and the alternations 47 per second. Accompanying these potential differences are the atomic weights.

\begin{tabular}{|c|c|c|c|c|c|c|c|c|c|}
\hline Bodies. & C & $\mathrm{Mg}$ & $\mathrm{Fe}$ & $\mathrm{Ni}$ & $\mathrm{Cu}$ & $\mathrm{Ag}$ & $\mathrm{Cd}$ & $\mathrm{Pt}$ & $\mathrm{Au}$ \\
\hline $\begin{array}{l}\text { Atomic Weight.... } \\
\text { Potential Dif..... }\end{array}$ & $\begin{array}{r}12 \\
640\end{array}$ & $\begin{array}{r}24 \\
700\end{array}$ & $\begin{array}{l}55.9 \\
850\end{array}$ & $\begin{array}{l}58.6 \\
850\end{array}$ & $\begin{array}{l}63.2 \\
870\end{array}$ & $\begin{array}{l}107.7 \\
900\end{array}$ & $\begin{array}{l}115 \cdot 5 \\
725\end{array}$ & $\begin{array}{l}194.3 \\
1000\end{array}$ & $\begin{array}{l}196.7 \\
1040\end{array}$ \\
\hline
\end{tabular}

Guye and Bron ${ }^{3}$ found that by using an E.M.F. of 20,000 volts and by keeping the electrodes at temperatures very near their melting points arcs of considerable stability were obtained. Under these conditions the period of extinction

1 Phys. Rev., 20, 374; 1905.

2 Ecl. Elec., 34, 305; 1902. Elektrot. ZS., 23, 956; 1902.

3 Archives des Sciences, 25, 453 and 549; rgo8. C. R., 146, rogo; and I47, 49; 1908 . 
was negligible and the "minimum potential difference" tended to a constant value whatever the nature of the metal, provided it was not easily melted. This voltage was in the neighborhood of 475 volts, the experiments being performed in air at a pressure of $40 \mathrm{~cm}$., the distance between the electrodes being $4 \mathrm{~mm}$., the current 0.1 ampere and the frequency 50 alternations per second.

The space between the electrodes with an arc between metals loses its conductivity more quickly than with the arc between carbons. This is shown by direct measurements of the conductivity and by the difficulty which is experienced in trying to maintain an alternating-current arc between metals. The experience of Edlund with the arc between silver electrodes has already been mentioned. Arons ${ }^{1}$ found that the arc between metals lost its conductivity very quickly. Duddell ${ }^{2}$ found that it was not possible to restart an arc between copper electrodes I/27000 sec. after it had been broken.

As has been stated Guye and Monash believed that the difficulty is proportional to the atomic weight of the metal, but it is hard to see how the atomic weight can directly affect the arc and the evidence is not strong for this view. Their data, for example, do not agree with the data of those who have worked with larger currents.

Granqvist believes that the difficulty of maintaining such an arc is due to the rapid conduction of heat by the electrodes. This, no doubt, has much to do with the phenomenon, and yet it is not a complete explanation, as is shown by the behavior of the alternating-current arc between carbons with different pressures of the surrounding

1 Wied. Ann., 57, I93; 1896.

2 Lond. Elec., 46, 3II; I900. 
gas. At atmospheric pressure it is easy to maintain such an arc on a circuit of I Io volts. In a vacuum where the pressure is less than $0.5 \mathrm{~mm}$. it is not possible to do so. ${ }^{1}$ The atomic weight has not changed and the heat conductivity has not increased, so that neither of the above explanations applies in this case.

All our knowledge of the arc would indicate that we must look to the cathode for an explanation of the difficulty. When this is sufficiently hot a current can be started. When it is not, no current will flow. In this case there are two things to be considered; first, how much heat was developed at the cathode during the preceding part of the cycle when it was the anode, and secondly, how fast it is conducted away. Granqvist called attention only to the second of these considerations.

With the carbon arc we not only have electrodes which are poor thermal conductors, but we have an exceptionally large anode drop with its corresponding large development of heat near the anode. The electrode reaches a high temperature while being the anode, and does not cool off rapidly during the change in the direction of the current. With the metal arc there is less heat developed and it is conducted away more rapidly.

With a carbon arc in a vacuum the heat is not conducted away more rapidly than with such an arc in air, but the anode drop is here very small and the carbon does not rise to a sufficiently high temperature during the time that it is the anode to enable it to start the arc when it becomes the cathode.

It is altogether possible that with the metal arc the ions between the electrodes become more quickly loaded with 1 Phys. Rev., 20, 374; Ig05. 
condensing vapor than in the carbon arc and that this may hinder the starting of the arc, but as yet we have no direct evidence of such action.

\section{Alternating-current Arc between Unlike Terminals. -} When an alternating current is passed between two unlike terminals, the current is in general greater in one direction than in the other. This is most noticeable when one terminal is a metal and the other carbon, but it is possible to observe it even with two carbon terminals, if they are of different sizes, or if one is cored and the other solid. ${ }^{1}$

Jamin and Maneuvier ${ }^{2}$ seem to have been the first to observe this. They found that when two carbons are of different sizes the current from the larger is greater than that in the opposite direction. The difference between the two currents is greater the greater the difference in the size of the carbons. They found also that with short arcs between a metal and carbon there is little difference between the currents in the two directions. The excess is greatest when mercury is used for one of the electrodes.

When an alternating-current arc is maintained between a carbon rod and a rotating carbon with an E.M.F. of 220 volts and 50 alternations per second about 30 per cent of the energy is rectified, the rod being positive with respect to the carbon. ${ }^{3}$

A large number of photographs which showed the curves of current and potential difference with the alternating-

1 Sahulka, Wien. Sitzungbr., 103, 2A, 925; 1894.

Gold, Wien. Sitzungbr., I04, 2A, 8I4; I895.

Von Lang, Wied. Ann., 63, I9I; I897.

Eichberg and Kallir, Wien. Sitzungbr., ro7, 2A, 657; 1898 .

${ }^{2}$ C. R., 94, 1615 ; 1882 .

${ }^{3}$ Sahulka, Elektrot. ZS., 29, 949; 1908. 
current arc between carbon and a metal were taken by Blondel ${ }^{1}$ by means of the oscillograph. He found that when there was inductive resistance in the circuit, the arc was extinguished for a longer time than when there was none. ${ }^{2}$ Fig. 36 gives a good idea of such curves. During

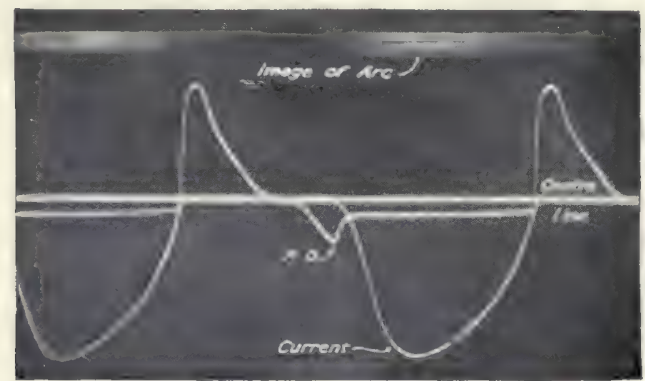

Fig. 36 .

one half period the current and potential difference curves are much the same as with ordinary alternating-current arcs. In the other the voltage rises to its normal value, but there is no corresponding current.

This phenomenon is the same as that described on page 69 where the peculiarities of the direct-current arc between unlike terminals were discussed, and the explanation of this is, of course, the same as that given in that discussion.

Alternating-current Arc in Other Gases than Air. There have been but few experiments performed on the alternating current in other gases than air. The results obtained from these are such as one would expect from those on the direct-current arc in different gases. Thus it has been observed that it is very difficult to maintain an

1 C. R., 128, 727; 1899.

2 See also Duddell and Marchant; Journ. Inst. Elec. Eng., 28, I; I899. 
alternating-current arc in hydrogen. ${ }^{1}$ For example, Upson found that he could not maintain such an arc between carbon terminals with a length of more than 0.03 in., the E.M.F. being 100 volts and the frequency 80 per second. Under similar conditions in coal gas an arc longer than 0.02 in. could not be maintained.

Oscillographs showing the current and voltage with alternating-current arcs in coal gas have been obtained by Morris. ${ }^{2}$ These show that the voltage rose to 370 volts before the current commenced.

Mercury Arc Rectifier. - As has been stated it is difficult to maintain an alternating-current arc in a vacuum, even when the electrodes are carbon. With any other electrodes it appears to be quite impossible to do so. The difficulty appears to be due to the fact that the terminal which is the anode during one half period does not become hot enough during that time to become the cathode when the E.M.F. is reversed, for a terminal must be very hot in order to become the cathode of an arc.

On the other hand a terminal can easily become the anode, even when it is cold. Use has been made of these two facts in devising an apparatus for rectifying alternating currents. The first device of this sort was made by Cooper Hewitt and called by him the static converter. This form can be used only with a three-phase current. It is shown diagrammatically in Fig. 37. The rectifier consists of a tube, $T$, with one mercury electrode at the bottom and three iron electrodes above. The iron electrodes are connected to the three circuits of a star-connected three-phase

1 Child, Phys. Rev., 20, 374; 1905.

Upson, Lond. Elec., 60, 58; 1907. Phil. Mag., (5), I4, I4I; I907.

2 Lond. Elec., 59, 707; 1907. 
generator; the mercury electrode is connected to the neutral point of the winding. At all times there will be one of the iron electrodes which is positive as compared with the mercury electrode. If an arc is once started from one of

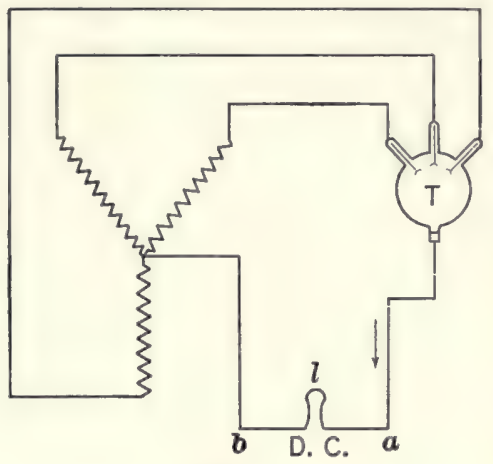

FIG. 37.

these to the mercury, the discharge easily changes to a second electrode when that in turn becomes positive. On the other hand no discharge can pass to an iron electrode at the time it becomes negative, since it can not become a cathode after having once ceased to be one. As a result the current will at all times be flowing from one of the iron electrodes to the mercury and will never flow in the opposite direction, so that between $a$ and $b$ there will be a continuous direct current. This can be used for lighting a lamp at $l$, for example, or in any other way desired.

For starting the rectifier a fourth iron electrode (not shown in the diagram) was introduced and a momentary high E.M.F. was produced between this and the mercury. The drop in potential through the rectifier was found by Cooper Hewitt to be about I4 volts. The percentage of 
loss would, therefore, be rather large on a rro-volt circuit. On circuits with higher voltage it would be correspondingly smaller.

An improved form of the converter which can be used with currents as large as 700 amperes has recently been brought out by the same inventor. ${ }^{1}$ This is made of a metallic envelope, through which the electrodes are inserted by way of porcelain tubes, the whole being subjected to artificial cooling. It has been found possible to use such a tube for a month without being reëxhausted while giving 200 kilowatts. This may well prove of value

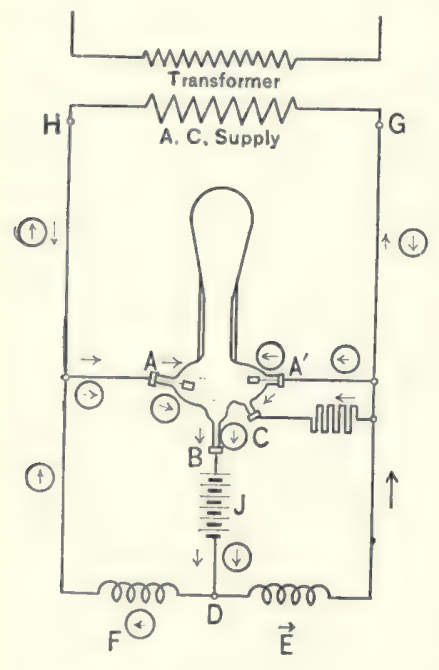

FIG. 38 . as a connecting link between an alternating-current supply system and direct-current motors, such as are largely used in railway service.

An ingenious modification of this for use on a singlephase circuit is described by Weintraub. ${ }^{2}$ This is shown in Fig. 38. The tube.has three terminals, $A, A^{\prime}$ and $B$. $B$ is a mercury terminal. The others may be either mercury, iron or carbon. Those shown in the tube are carbon. $C$ is an auxiliary terminal used in starting the arc. $A$ and $A^{\prime}$ are connected to the terminals of an alternating-current supply. $\quad E$ and $F$ are coils having considerable inductance.

1 Elec. World, 59, 628; I912.

2 Phil. Mag., (6), 7, 122; I9०4. 
If the tube is rocked so that the mercury in $B$ is brought into momentary contact with that in $C$ and then separated an arc is started. At the moment that this occurs either $A$ or $A^{\prime}$ will be positive with respect to $B$ and a current will flow from whichever is positive to the cathode $B$. If the auxiliary arc is not maintained, the current from $A$ or $A^{\prime}$ will continue until $A$ and $B$ are at the same potential. If there were no self-induction in the circuit the arc would then cease, but with the inductance coils at $E$ and $F$ the arc will continue even without the auxiliary arc, as will be seen from the following.

Let us assume, for example, that $A$ is at a higher potential than $B$ at the moment contact is broken between $B$ and $C$. Current will then flow from $A$ to $B$ down to $D$ and through $E$ back to $G$. Only a very small amount will flow through $F$ because of its large inductance. As soon as this current begins to decrease the energy stored in the coil $E$ raises $A^{\prime}$ to a higher potential than $B$ and current flows from $A^{\prime}$ to $B$. This occurs while the current from $A$ is decreasing but before it has stopped, so that at this moment current is flowing to $B$ from both $A$ and $A^{\prime}$, as is indicated in the diagram by the arrows without circles.

As the direction of the E.M.F. is reversed the current will cease to flow from $A$, but it will continue to flow from $A^{\prime}$ because of the E.M.F. induced by the coil $E$, and before this E.M.F. has ceased the transformer will cause $G$ to be positive and the current will continue to flow from $A^{\prime}$. It can not flow to $A$ because a cold terminal can not become the cathode of an arc. It will, therefore, flow through $B$ and $F$ and there will be the same sequence of events on this side as had previously occurred on the other, the current for a brief interval flowing from both $A^{\prime}$ and $A$ 
through $B$ and the inductance $F$, as indicated by the arrows with circles.

The efficiency of this rectifier is good even on a hundredvolt circuit, and becomes higher as the voltage is raised. Norden ${ }^{1}$ states that the efficiency of such rectifiers giving Ioo volts is 80 per cent for currents varying from Io to 30 amperes, while the efficiency of the rotary converter giving the same voltage is only 54 per cent for Io amperes and 62 per cent for 30 amperes. In general, he says, the static rectifier is more efficient than the rotary converter when less than 5 kilowatts are being used.

The "return" current, that is, the current passing through the tube in a direction opposite to the main current, is ordinarily but a few millionths of an ampere. ${ }^{2}$ It increases slightly as the voltage is increased and very greatly when the current is increased, so that the tube becomes hot.

As has been stated it is not possible to pass an alternating current through a vacuum tube having mercury for one of the electrodes. This is true when there are only two electrodes or when there is no inductance in the circuit, but evidently there is no reason why the mercury arc rectifier, as described above, should not be used to give light, and in fact such a lamp is now being sold. When used for this purpose it is made longer than when used as a rectifier and the voltages are so arranged that the greater part of the energy is used in the tube instead of in the outside circuit. Such an arrangement is a mercury vapor lamp for alternating circuits.

It has been found by Bedell ${ }^{3}$ that partial rectification

1 Allgem. Elek. Ges. ZS., Feb., Igro.

${ }^{2}$ Schultze, Elektrot. ZS., 3I, 28; 19 IO.

3 Elec. World, 40, 408; r902. 
may be produced by placing an alternating-current arc between carbon terminals in a magnetic field. One end of the arc can then be made to alternate between two terminals which are very near each other but not in contact. As a result one of these terminals will receive nearly all of the positive current and the other nearly all of the negative. The terminals can then be connected so as to produce a direct current through part of the circuit. 


\section{CHAPTER VII.}

\section{Photometry of the Electric Arc.}

THE chief use of the arc is to givelight and its commercial value depends on the amount and color of its light. These subjects will be considered briefly in the following chapter, but they belong more properly to books on photometry and spectroscopy and the reader is referred to such books for their more adequate treatment.

There are three difficulties met with when making measurements of the candle power of electric lamps. The first and most radical one is due to the difference between the color of the arc and that of the light standards in common use. Nearly all other forms of illumination are similar in color to the candle and can be compared with it, but this can not be said of the arc. The open arc is distinctly bluer. The enclosed is somewhat less blue due to the absorption by the glass, but it is still bluer than the candle. The flaming arcs vary greatly, but none of them have the same color as the candle, and the mercury arc is less like the candle than any of the others.

Not only is it difficult for an observer to compare two unlike colors in a photometer, but different observers arrive at results differing often by many per cent. The ideal method would be one giving relative values for practical purposes, such as reading a paper or seeing an obstacle in the road, but unfortunately we have no way of making definite comparisons of this sort. 
A second difficulty is due to the continuous fluctuation of the arc. An arc between the better kinds of carbons can be kept fairly steady by constant regulation, although even then sudden changes are apt to occur. But even if satisfactory measurements are made, one has no very definite knowledge of the candle power of the lamp when run under commercial conditions where the regulation of the lamp is not perfect. Fortunately, where cored carbons are used for the anode, this difficulty is not as great as it is with solid carbons.

A third difficulty is due to the irregular distribution of the light. There is no form of artificial light which is equally luminous in all directions, but the common form of the arc has the disadvantage that there are two directions in which the mechanism of the lamp obstructs the light. Moreover the distribution of light from a directcurrent arc is not symmetrical about these two ends. This can well be seen by referring to Fig. 39 taken from the data of Fleming and Petavel. ${ }^{1} O$ is the arc. The intensity of light in any direction is indicated by the curved line and is proportional to the distance of this line from the $\operatorname{arc} O$. Again the center of the arc is usually at one side of the center of the carbons and wanders from one place to another. As a result the distribution of the light about a vertical axis is unsymmetrical and the intensity in any direction will vary from time to time.

Because of the irregular distribution of light from the arc, it means nothing to say that an arc has a certain candle power, unless some definite direction is also given. An arc light of Iooo candle power might be a very good light or a poor one, depending on the direction in which the

1 Phil. Mag., (5), 4I, 355; 1896. 
light was measured. Because of this irregular distribution the average intensity over a part or the whole of the region illuminated is often given. If the average intensity in all directions is given, it is called the mean spherical candle power (abbreviated to m.s.c.p.). Since the part of the light which is thrown below the horizontal plane passing through

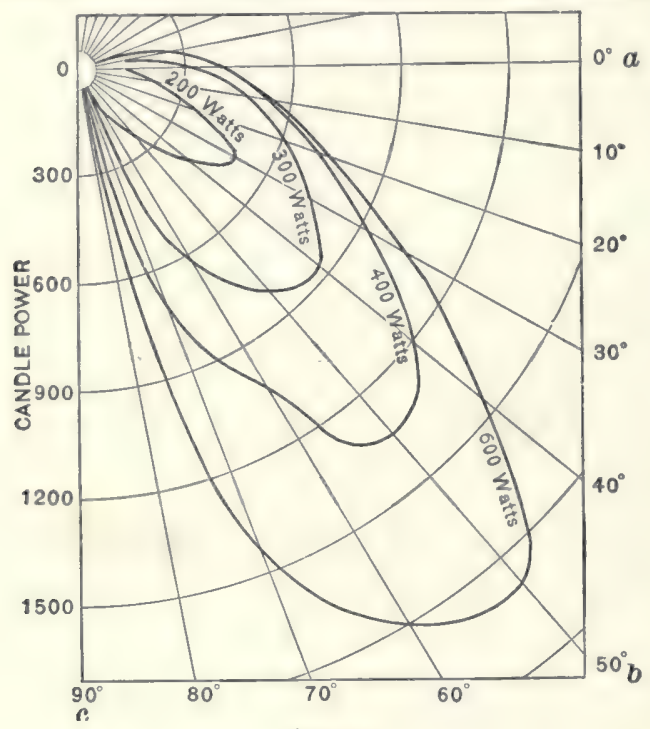

FIG. 39 .

the light is all that is useful in the majority of cases, measurements are often made of this part alone and the average of this is called the mean hemispherical candle power (m.h.s.c.p.).

Some of the measurements given in the following pages are taken from German sources and are given in hefner units. One hefner unit is equivalent to 0.88 English candle.

Distribution of Light. - With the common form of the carbon arc the greater part of the light comes from the 
crater of the anode. The cathode gives a small amount, but obstructs more than it gives out, while the gas between the carbons absorbs as much as or perhaps more than it gives out. The first person to give any statement concerning the distribution of light from the arc which could be put in simple form was Trotter. ${ }^{1}$ His statement was that the intensity of light in any direction was approximately proportional to the component of the area of the crater which was perpendicular to and visible from that direction. Thus above $a$ in Fig. 39 little of the crater would be visible, because of its concave shape, and what is visible would have but a small component perpendicular to the line drawn to $a$. As seen from $b$ there would be a large component perpendicular to the line drawn to $b$, while at $c$ it would be largely hid by the cathode. This statement is, of course, only approximately correct, since some light comes from the cathode and some from the vapor, but Trotter showed that this was only a few per cent of the total amount.

The value of an arc for lighting purposes depends to a very great extent on the distribution. For example, the distribution shown in Fig. 39 is undesirable, since almost no light is thrown immediately below the lamp and very little in a horizontal direction. The first defect would make the lamp a poor one for indoor lighting, since there would be a considerable dark region below the lamp. However, such lamps are not often used for indoor work and the more serious defect is the second one. Near the lamp there will be light enough for street lighting in any case, but as the distance increases the amount of light rapidly diminishes, both because the distance is greater

${ }^{1}$ Lond. Elec., 28, 687; and 29, II; 1892. 
and because the direction is nearly horizontal. For example, if a lamp giving the distribution shown in Fig. 26 were hung at a height of $\mathrm{I}_{5} \mathrm{ft}$., the light striking the ground at a distance of $150 \mathrm{ft}$. would make an angle with the horizontal of less than 6 degrees, and the candle power in this direction is not more than a fourth of what it is in a direction making an angle of 50 degrees with the horizontal.

Effect on Candle Power Produced by Length of Arc, Current, Etc. - From the statement given above concerning the distribution of light it follows that any cause affecting the amount of the crater that is visible from any given direction affects the intensity of light in that direction. Thus the shorter the arc the more the light from the crater is cut off by the cathode. One would expect from this that the longer the arc the greater would be the candle power. There is the more reason to expect this, since there is more luminous vapor with the longer arcs. It has been found, however, that the candle power does not increase indefinitely as the arc is lengthened, but reaches a maximum and then decreases with greater length. Thus Ayrton found the maximum candle power to be with lengths of about $4 \mathrm{~mm}$. Mrs. Ayrton plotted curves taken from the data of Blondel. ${ }^{1}$ These are reproduced in Fig. 40. The numbers near the curves indicate the diameters of the electrodes. Those above the dash indicate the diameter of the anode in millimeters; those below, the diameter of the cathode.

From this it would appear that there is a maximum candle power at about $6 \mathrm{~mm}$. of length. Mrs. Ayrton believed the decrease with greater lengths to be due to the absorbing power of the vapor between the terminals.

${ }^{1}$ Mrs. Ayrton's "Electric Arc," p. 33.3. Ecl. Elec., 10, 289, 496, and 539; 1897 . 
Since the intensity of light becomes less with these lengths

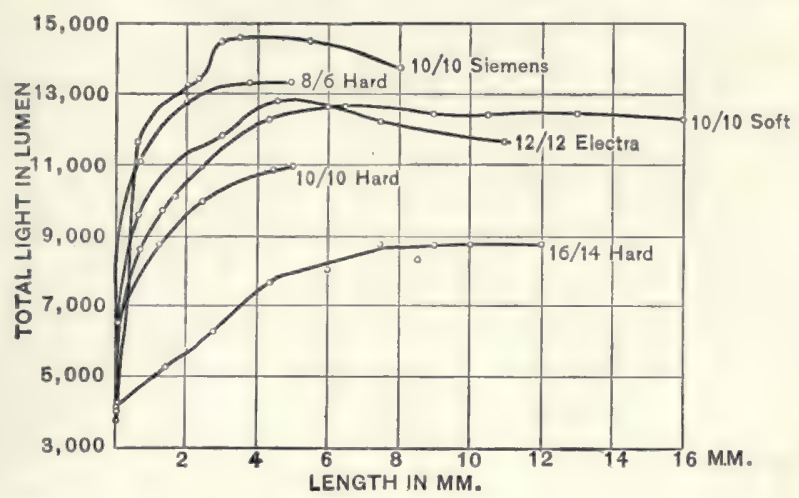

FIG. 40.

and the power consumed becomes greater, the ratio between the two becomes rapidly smaller with arcs more than a few millimeters long.

Fig. 40 illustrates also the difference between different kinds of carbons and shows how impossible it is to make any definite statement concerning the candle power of arc lights, or to give any formula which will hold with different carbons. No two kinds of carbons vary in the same way. One might almost say that no two pairs

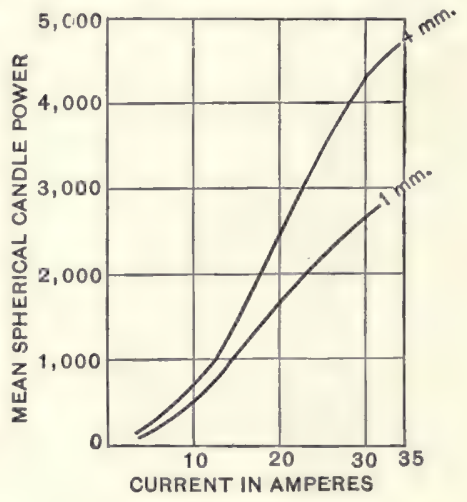

FIG. 4I. of carbons of the same make show the same results.

Measurements were made by Ayrton with different cur- 
rents keeping the length of the arc constant, and by Blondel ${ }^{1}$ with the voltage constant. Fig. 4I reproduces two of the curves given by Ayrton. ${ }^{2}$ As may be seen, the increase in light is nearly proportional to the current flowing. Rey measured the candle power with currents up to 250 amperes and states that the light increases more rapidly than the current. $^{3}$

The larger the carbons the less the candle power, due to shading of the light from the crater by the carbons. ${ }^{4}$ It has also been found that there is a loss of candle power when the pressure of the surrounding gas is more than one atmosphere. This is due to the presence of fumes of nitric acid. ${ }^{5}$

Intrinsic Brightness of Crater. - For many purposes the usefulness of a light depends not only on the total amount of light, but also on the amount given per unit area of the luminous surface. A concentrated light is often blinding and may be injurious to the eye. In this respect all forms of the arc are far from being ideal and the carbon arc is the worst of all lights. Blondel states that the intrinsic brightness of the crater is 160 candles per sq.mm. Petavel ${ }^{6}$ found a value of 147 candles per sq.mm. for a current density varying from 0.06 to 0.26 ampere per sq.mm. This is something like 200,000 times the brightness of a candle flame.

Flaming Arcs. - The previous discussion applies to the older form of arc where by far the greater part of the light comes from the anode. In the flaming arcs the chief

1 Ecl. Elec., 10, 297; 1897.

2 Mrs. Ayrton's "Electric Arc," p. 366.

${ }^{3}$ Ecl. Elec., 32, I1I; 1902.

4 Schreihage, Centralbl. f. Elektrot., ro, 604; 1888.

s Wilson, Astrophys. Journ., 2, 21 2; 1895.

6 Proc. Roy. Soc., 65, 469; 1899. 
source of light is the long luminous vapor. Because of the high conductivity of this vapor, it is possible to have very long arcs and the candle power is approximately proportional to the length of these arcs.

The distribution of light is much more symmetrical than with the more common forms. In some cases this is a disadvantage, as when the light is needed beneath the lamp. On this account the carbons are sometimes placed nearly parallel and above the arc as shown in Fig. 42, the

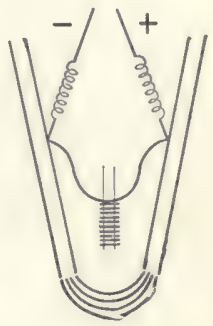

FIG. 42. arc being kept below by means of a magnet. This is the same plan as that of the "Carbone" lamp. ${ }^{1}$

The amount of light depends on the amount of substance added to the carbon. For example, the candle power with different percentages of calcium fluoride has been studied by Wedding ${ }^{2}$ and his results are shown in Fig. 43. The arc with which he worked was one between converging carbons with a reflector above. In each case there was a current of 9 amperes and a potential difference of 45 volts. The amount of added mineral is given with each curve.

- The efficiency of the flaming arc varies, of course, with different makes of carbons and with different kinds of lamps. Sharp ${ }^{3}$ states that Blondel's flaming arc requires between 0.I 6 and O.I 8 watt per mean lower hemispherical candle power, which is less than one-fourth of that of the open carbon arc. Marchant ${ }^{4}$ states that the yellow

1 Lond. Elec., 57, 51, and 129; 1906.

2 Elektrot. ZS., 23, 702; 1902.

3 Trans. Intern. Elec. Cong., St. Louis, 2, 765; I904.

4 Elec. World, 56, 216; 19ro. 
flaming arc requires 0.144 watt per mean hemispherical candle power, and the white flaming arc 0.338 watt. Further data will be given in the tables at the end of this chapter, but for an adequate knowledge of the photometry of these lamps reference must be made to the articles constantly appearing in the technical journals. ${ }^{1}$

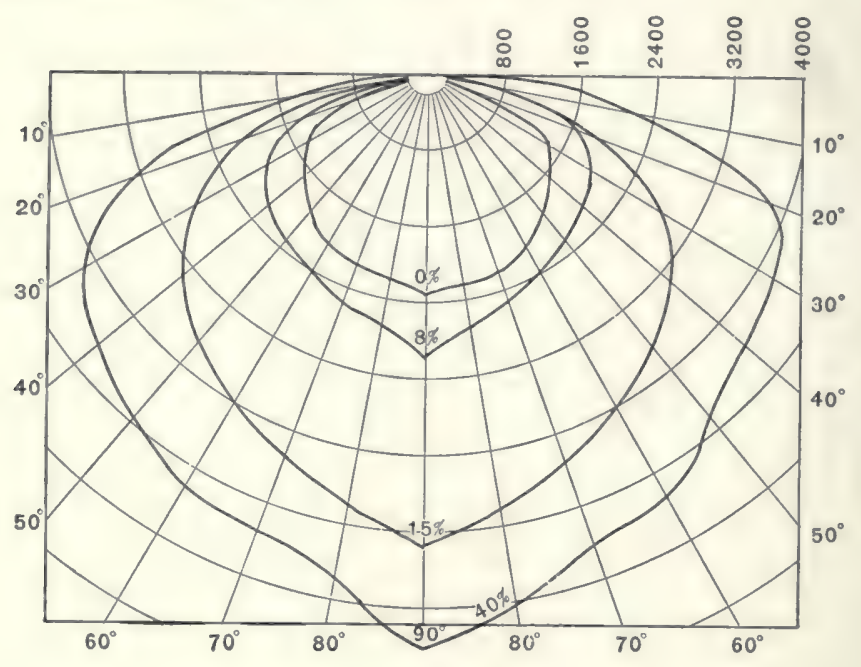

FIG. 43 .

Metal, Including Mercury, Arcs. - As far as I have been able to learn no measurements have been made on the candle power of arcs between metals in air. ${ }^{2}$ None of these

1 See also Bloch, Elektrot. ZS., 30, 703; I909.

Georges, Elektrot. ZS., 30, 595; r9o9.

Heyck, Elec. World, 54, 863; I909.

Elektrot. ZS., 30, 1055; I909.

Monash, Elektrot. ZS., 30, 34I; r9o9.

${ }^{2}$ This does not include arcs having one terminal an oxide of metal such as the magnetite arc. 
have ever been used commercially, and there is little object in getting their candle power for any other purpose.

The candle power of the mercury arc has been examined by Kuch and Retschinsky. ${ }^{1}$ Their measurements were made with a mercury arc in a quartz tube approximately $27 \mathrm{~cm}$. long. The comparisons were made with standard

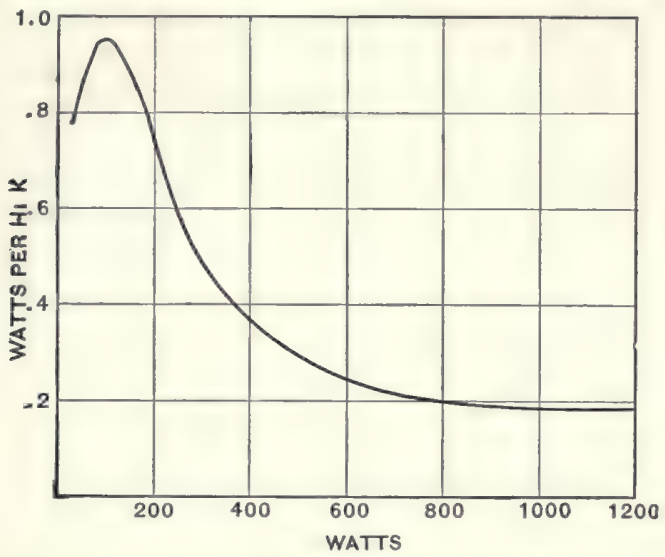

FIg. 44.

incandescent lamps by means of a flicker photometer. They found the intensity of light with a current of 1.9 amperes and voltage of 27 volts to be 58 candles ( 66 hefner units). With 3.85 amperes and 304 volts the candle power was 6270 . In Fig. 44 the relation is shown between the watts used and the watts per hefner unit.

They found also that the energy of the ultra-violet light per watt of power supplied also passed through a maximum and decreased in a manner similar to that of the visible rays.

Alternating-current Arcs. - The chief difference between the alternating- and direct-current arcs as far as light-

1 Ann. d. Phys., 20, 568; 1906, and 22, 852; 1907. 
giving properties are concerned is that with the latter the greater part of the light comes from only one of the ter-

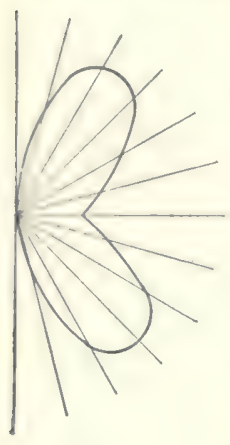

FIG. 45 . minals, namely the anode, while with the former, both terminals give the same amount of light, the anode alternating from one to the other terminal. The distribution of light is, accordingly, that shown in Fig. 45 .

With ordinary frequencies the light from the alternating-current arc appears to the eye to be continuous, while with smaller frequencies the flickering becomes noticeable. Thus Georges ${ }^{1}$ found that with 60 periods per second the flickering was not noticeable, while with 40 periods per second it was very disagreeable.

While this is the appearance presented to the eye, the actual amount of light changes very appreciably during an alternation, the candle power corresponding much more closely to the current flowing at each instant than might be expected when one remembers that the crater must be heated up and cooled off with each alternation. This is shown by curves in Fig. 46. In this figure the curve marked $a$ represents the potential difference, $b$ the current, $c$ the watts, and $d$ the candle power. During the first part of the alternation the photometer was illuminated by the light of the anode. During the second part this carbon had become the cathode and was giving much less light. ${ }^{2}$ Duddell $^{3}$ found that when an alternating current was

${ }^{1}$ Elektrot. ZS., I6, 548; 1895 .

2 See also Leonard, Ecl. Elec., 42, 24I; 1905.

${ }^{3}$ Lond., Elec., 46, 270; r900. 
superimposed on a direct current, changes in the light could be observed when the change in the current was only 3 per cent of the total current and when there were 4300 alternations per second.

The alternatingcurrent arc is not so efficient as the one with direct current. ${ }^{1}$ A comparison of the two for different currents is

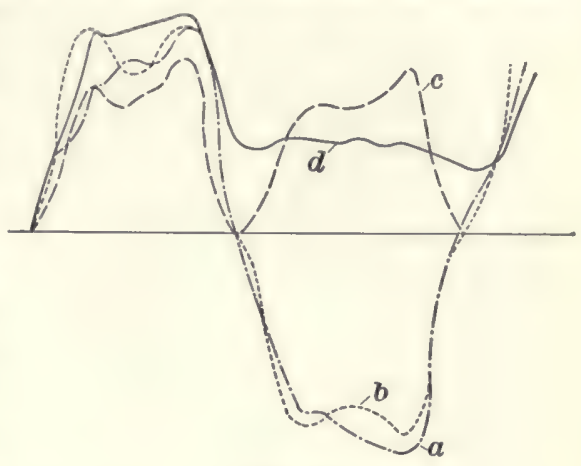

FIG. 46. given in Fig. 47. Matthews gave the candle power per watt for the direct-current enclosed arc as 0.76 , for the alternating-current arc without reflector as 0.53 and with reflector as 0.68 .

This form of arc is influenced by length, amount of current and size of carbons much the same as the direct-current arc is. Thus the watts per candle power become greater as the arc becomes longer. This is shown in Fig. 48, taken from the work of Fleming and Petavel. The candle power is less the larger the diameter of the carbon used and increases approximately as the current. ${ }^{2}$

The candle power per watt has been found to depend both on the wave form of the current and on the number of alternations per second. Rossler and Wedding ${ }^{3}$ found

${ }^{1}$ Fleming and Petavel, Phil. Mag., (5), 4I, 359; I896.

Dyke, Phil. Mag., (6), 10, 216; I905.

Elektrot. ZS., 23, 6r5; 1902.

2 Blondel and Jigouzo, Elec. World, 29, 232; 1897.

s Elektrot. ZS., 15, 315; I894. 


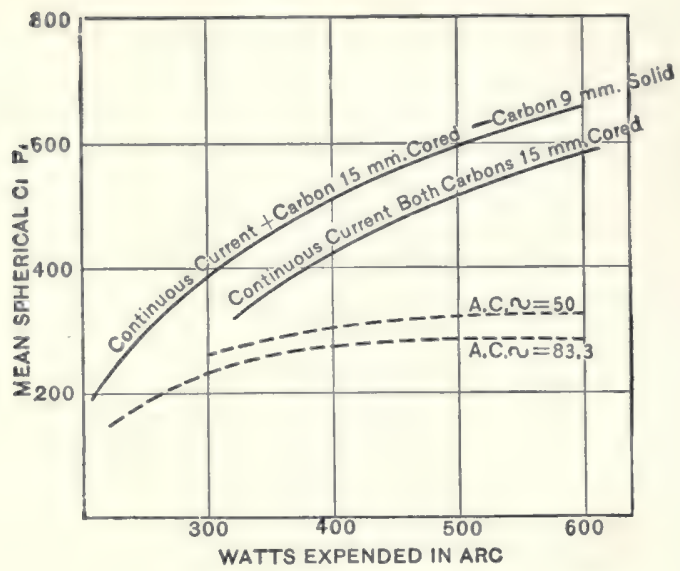

FIG. 47.

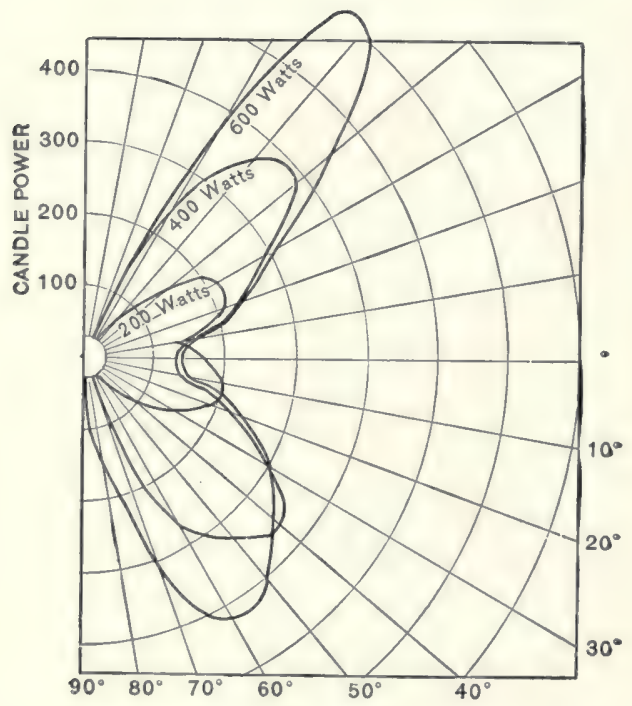

Fig. 48. 
that with a pointed form there was 0.7 Io hefner unit per watt and that for a flat curve there were I.024 hefner units. They also found that the candle power decreased as the number of alternations increased.

Comparison of Different Kinds of Arcs. - A comparison of candle power of different arc lamps has been made by Blondel ${ }^{1}$ and his results are given in the following table.

\begin{tabular}{|c|c|c|c|c|c|c|c|}
\hline \multirow[b]{2}{*}{ Type of lamp. } & \multirow[b]{2}{*}{$\begin{array}{l}\text { 鄫 } \\
\text { 总 }\end{array}$} & \multirow[b]{2}{*}{$\frac{\stackrel{8}{0}}{\frac{0}{0}}$} & \multicolumn{2}{|c|}{ Watts. } & \multirow{2}{*}{ 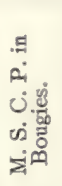 } & \multirow{2}{*}{ 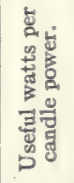 } & \multirow{2}{*}{ 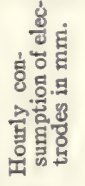 } \\
\hline & & & 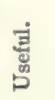 & 营 & & & \\
\hline 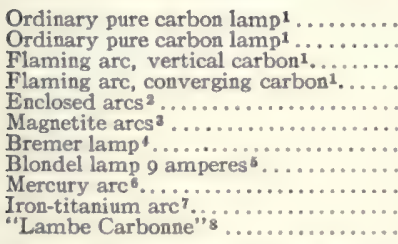 & $\begin{array}{l}9 \\
9 \\
9 \\
9 \\
6.8 \\
3.5 \\
9 \\
9.1 \\
3.5 \\
3.5 \\
10\end{array}$ & $\begin{array}{l}40 \\
35 \\
40 \\
45 \\
70 \\
9 \mathrm{I} \\
48 \\
43 \\
80 \\
48.3 \\
90\end{array}$ & $\begin{array}{l}360 \\
315 \\
360 \\
405 \\
476 \\
320 \\
432 \\
391 \\
280 \\
169 \\
900\end{array}$ & $\begin{array}{r}495 \\
330 \\
495 \\
495 \\
768 \\
385 \\
495 \\
500 \\
385 \\
385 \\
1100\end{array}$ & $\begin{array}{r}700 \\
540 \\
910 \\
2000 \\
329 \\
400 \\
4814 \\
4800 \\
770 \\
700 \\
1070\end{array}$ & $\begin{array}{l}0.514 \\
0.583 \\
0.396 \\
0.202 \\
1.45 \\
0.80 \\
0.088 \\
0.081 \\
0.362 \\
0.242 \\
0.84\end{array}$ & $\begin{array}{l}\mathrm{I} 6-\mathrm{I} 7 \\
\mathrm{I} 6-\mathrm{I} 7 \\
27.5 \\
34-42.5 \\
\mathrm{I} \cdot 5-2 \\
\mathrm{I}-2 \\
35-45\end{array}$ \\
\hline
\end{tabular}

Alternating-current Lamps.

\begin{tabular}{|c|c|c|c|c|c|c|c|}
\hline Ordinary pure carbon $1 .$. & 9 & 30 & 270 & 330 & 350 & 0.772 & \\
\hline Ordinary cored carbon $9 . . .$. & 15 & 35 & 480 & 555 & 470 & 1.02 & I5-I6 \\
\hline Flaming arc, vertical carbon......... & 9 & 30 & 270 & 330 & 700 & 0.386 & 30 \\
\hline Flaming arc, converging carbon ....... & 9 & 45 & 405 & 495 & 2000 & 0.202 & $35-45$ \\
\hline Enclosed $\operatorname{arcs}^{2} \ldots \ldots \ldots \ldots \ldots$ & 6.6 & 70 & 462 & 726 & 314 & I. 47 & $x-2$ \\
\hline Blondel lamp ${ }^{\circ} . . . . . . .$. & Io & 35 & 255 & 370 & 1890 & 0.135 & $15-16$ \\
\hline Carbo-mineral lamp ${ }^{5} . . . . . . . . . .$. & 8 & 33 & 225 & 372 & 1000 & 0.225 & $15-16$ \\
\hline
\end{tabular}

1 Zeidler, Elektrotechnishe Verein von Berlin, Dec, 23, I902.

2 Matthews, second report, p. 30, and third report, p. I7, N. E. L. A. A.

M. W. Holmes, Elec. World, 43, I053; I904.

- Czudnochowski, Vertrag der Deutschen Phys. Gesellschaft, I903, No. 7 .

- Essais des Laboratoire de la Soc. Auer de Paris.

B Westinghouse circular.

7 Ladoff Ecl. Elec.

8 Monash, Elektrische Beleutung, p. I79.

- Essais des Laboratoire central d'Electricite de Paris.

1 Trans. Intern. Elec. Cong., St. Louis, 2, 755; 1904. Ecl. Elec., 52, 68; 1907. 
A more recent comparison of direct-current arc lamps has been given by Ryan $^{1}$ in which the following lamps were compared.

(I) The carbon open arc, 9.6 amperes, 50 volts, consuming in practice from 450 to 500 watts, commercially rated at 480 watts, equipped with clear globe and no reflector. Electrode life of from I6 to 18 hours. This lamp is normally designated as 2000 c.p., which, however, is regarded as merely a trade name.

(2) Enclosed carbon arc, requiring 6.6 amperes and from 70 to 75 volts, consuming in practice from 450 to 500 watts, commercially rated at 480 watts, equipped with light opal inner, clear outer globe and street reflector. Electrode life of from Ioo to I 50 hours.

(3) Magnetite arc, requiring 4 amperes and from 75 to 80 volts, consuming in practice from 300 to 320 watts, commercially rated at 310 watts, equipped with clear outer globe, internal concentric diffuser and magnetite electrode. Electrode life of from I 50 to 200 hours.

(4) Magnetite arc, requiring 6.6 amperes, 75 to 80 volts, consuming in practice from 495 to 530 watts, commercially rated at 5 10 watts, equipped with clear globe, internal concentric diffuser and magnetite electrode. Electrode life of from 75 to I25 hours (large unit).

(5) Boston flaming arc, requiring from 6.6 amperes and from 75 to 80 volts, consuming in practice from 495 to 530 watts, commercially rated at 5 10 watts, equipped with 26-in. concentric diffuser and light opal outer globe. Electrode life of from 20 to 25 hours.

The following are the data which he gives concerning these.

1 Paper presented before the N. E. L. A., at St. Louis, I9Io. 


\begin{tabular}{c|c|c|c|c|c|c|c|c|c}
\hline Lamps. & Amps. & Volts. & Watts. & H.S.C.P. & $\begin{array}{r}\text { Watts } \\
\text { per }\end{array}$ & S.C.P. & $\begin{array}{r}\text { Watts } \\
\text { per } \\
\text { S.C.P. }\end{array}$ & $\begin{array}{c}\text { Total } \\
\text { lumens. }\end{array}$ & $\begin{array}{l}\text { Down- } \\
\text { ward } \\
\text { lumens. }\end{array}$ \\
\hline Open Arc ..... & 9.6 & 50 & 480 & 813 & 0.59 & 540 & 0.89 & 6,800 & 5,100 \\
Enclosed Arc.. & 6.6 & $70-75$ & 480 & 505 & 0.95 & 310 & 1.55 & 3,900 & 3,180 \\
Luminous Arc. & 4.6 & $75-80$ & 310 & 523 & 0.59 & 276 & 1.12 & 3.500 & 3,300 \\
Luminous Arc. & 6.6 & $75-80$ & 510 & 1328 & 0.38 & 718 & 0.71 & 9,000 & 8,300 \\
Flame Arc.... & 6.6 & $75-80$ & 510 & 2964 & 0.17 & I821 & 0.28 & 23,000 & 18,600 \\
\hline
\end{tabular}

The curves showing the distribution of light for these arcs are given in Fig. 49. While the total quantity of light

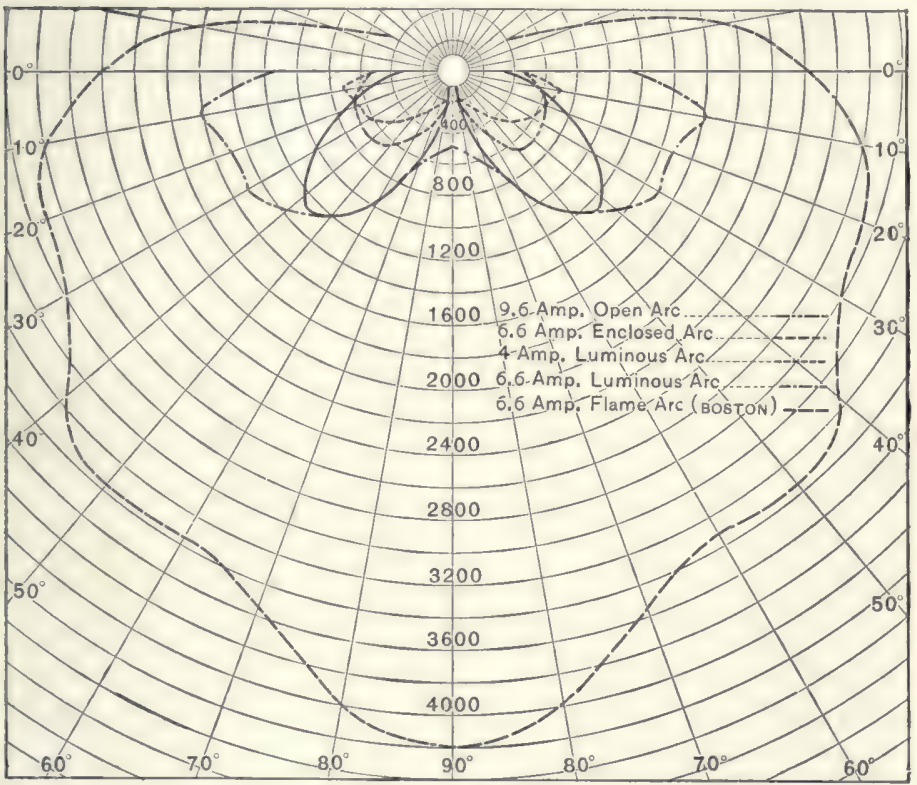

FIG. 49.

emitted by the open carbon arc is greater than that for either the closed arc or for the first form of the magnetite arc, it will be seen from the curves that the amount sent in a horizontal direction is less for this arc than for any of 
the others, and the value of a lamp for street lighting depends very largely on the amount sent in this direction. This is partly responsible for the fact that the open arc has been superseded to a very great extent by other forms.

The figures for the flaming arc show a very low value of the watts per candle power and also a very desirable distribution of the light. In the first of these respects it excels any other form of light.

In I908 a report was submitted to the German Government ${ }^{1}$ in which they gave the light intensity in hefner units per kilowatt for different sources of light as follows:

Common form incandescent filament lamps.......... 300

Improved incandescent filament lamps............ 500

Nernst and tantulum incandescent lamps.......... 500

Osram and tungsten .................. from 700-1000

Alternating-current arc between cored carbons........ 800

Direct-current arc between cored carbons........... I000

Direct-current flaming arc.................. 2000

Mercury vapor arc....................... 1600

Mercury vapor arc in quartz tube............. 3000

Spectrum of the Arc. - The spectrum of the terminals of the arc is, of course, the continuous spectrum of an incandescent solid and does not differ from that of other incandescent solids.

Unfortunately, no such simple statement can be made concerning the spectrum of the gaseous part. Instead of some simple spectrum of carbon monoxide, for example, with an occasional line due to some impurity in the carbon, there is one so complicated that it is not possible to determine the cause of all the lines. There are not only lines due to all the impurities which would be expected, but many due to impurities which would not be expected.

1 La Revue Elec., Jan. I5, I909. 
In addition the spectra of different parts of the arc show decided differences. The arc has indeed been divided into two or three different regions by those who have examined its spectrum. This is more or less arbitrary but will help us to gain some idea of the colors in different parts. For example, Foley ${ }^{1}$ divided the carbon arc into three parts, as shown in Fig. 50. The first region is violet, the second is blue and the third yellow. At times the first region extends entirely across from the anode to the cathode. At other times it extends only part way as shown in the diagram.

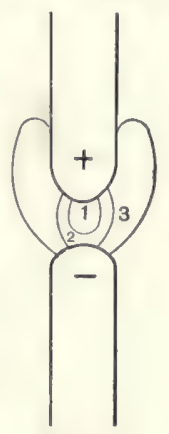

FIG. 50.

The spectra of all parts show a great many lines. Many of these belong to the carbon spectrum, but even when the purest carbon is used, there are also many lines which can be identified as lines of various metals. There are also many lines about which there is still more or less dispute. It can not be definitely stated whether these are carbon lines or metallic lines which do not ordinarily show in the spectrum, but are brought out by the peculiar conditions of the arc.

The number of lines in the spectrum of the first region is much greater than in those of the other two. This is due chiefly to the fading out of carbon bands in the second and third regions, but the lines of metals also show the same tendency. Foley found the third part to be composed largely of vapor of calcium and iron.

Miss Baldwin ${ }^{2}$ found that the carbon bands showed more clearly at the positive pole and the metallic lines at the

1 Phys. Rev., 5, I29; I897.

2 Phys. Rev., 3, 370; 1895. 
cathode. Hull ${ }^{1}$ makes a similar statement for currents of only a few amperes, but states that with the hissing arc the metallic spectra appeared at both poles.

When metals were introduced into the arc, Miss Baldwin found that the more positive ones, such as potassium, sodium, lithium, barium, strontium and calcium appeared at the cathode more brightly than those less electropositive, such as cadmium, zinc, copper and silver.

One of the most important observations on the spectrum of the arc, provided it is correct, was made by Lenard. ${ }^{2}$ He states that the arc consists of different layers and that different series of lines of the spectrum of a given element appear in these different layers. Thus, if sodium is introduced into the arc, only the principal series of the spectrum appears in the outside layer. The first subordinate series of lines appears in an inner layer of vapor and the second subordinate series in a layer within this. An absorbing medium which cuts out all of the lines except the principal series, shows all of the vapor of the arc. A medium leaving only the first subordinate series will show an inner cone, but not the whole luminous vapor.

Lenard's explanation is that the atoms of an element are acted on differently in different parts of the arc and that each series is produced by one particular method of excitation. The experiment itself has been verified by Puccianti. $^{3}$ On the other hand Kayser ${ }^{4}$ does not agree with Lenard but thinks that what he observed was due to varying intensity of the different lines in different parts of the arc.

1 Astro-phys. Journ., 28, 59; I908.

2 Ann. d. Phys., I1, 636; I903.

3 N. Cimento, (5), 13, 268; 1907. Phys. ZS., 8, 463; 1907. ,

- Sz. wiss. Phot., 6, 68; 1908. 
The matter is one of much importance from a theoretical point of view, as almost nothing is known concerning the reason why very different spectra are given by the same element under different circumstances. A knowledge of the cause for this difference would undoubtedly throw much light on the construction of the atom and its behavior when disturbed by electrical discharge. Even if the phenomena are not as simple as was supposed by Lenard, there still is no question but that the spectra of elements differ in different parts of the arc and a further study of these variations may throw light on this question.

An examination of the spectra of the arc under different pressures has been made by Humphreys. ${ }^{1}$ He found that as the pressure increased the width of the lines increased, some, however, more than others. The metallic lines were somewhat displaced; the carbon lines were not.

The statement has been made by Steinmetz ${ }^{2}$ that in general the spectrum of the arc is that of the cathode material. This, however, would appear to be true only when the anode is not heated to a temperature sufficiently high to vaporize it. With a carbon anode in air, or with the majority of metals in air this does not appear to be the case. Cady and Arnold, for example, found that with ordinary currents the spectrum of the anode was very apparent. $^{3}$

1 Astro-phys. Journ., 26, I8 and 36; I907.

2 Trans. Intern. Elec. Cong., St. Louis, 2, 711 ; r904.

${ }^{3}$ Further work has been done by the following:

Snow, Phys. Rev., 1, I08; 1893.

Duffield, Astro-phys. Journ., 26, 375; I900.

Hallwachs, Ann. d. Phys., 13, 38; 1904.

Crew, Astro-phys. Journ., 20, 274; I904.

Crew and Spence, Astro-phys. Journ., 22, I99; r905. 


\section{CHAPTER VIII.}

Use of ArC iN Wireless Telephony.

Whispering Arc. - The electric arc has been used in two different systems for wireless telephony. The first of these had its origin in a discovery made by Simon in $1898 .{ }^{1}$ While working with an arc, he observed that it gave a chattering noise whenever an induction coil in a neighboring room was operated. He found, on examination, that for several feet the feed wires for the induction coil were
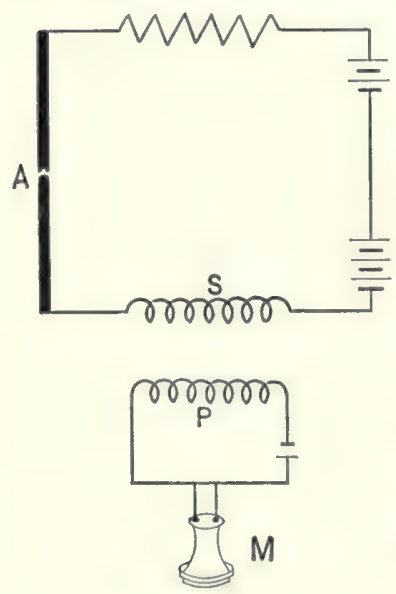

FIG. 5I. parallel to those running to the arc. With each variation of the current in the induction coil there was an induced current in the arc circuit, and with each fluctuation of the current in the arc circuit there was an expansion and contraction of the vapors of the arc which sent out sound waves.

This suggested to him the idea that instead of the induction coil the transmitter of a telephone could be used and the arc be made to reproduce the sound of the words spoken into the transmitter, and on trying the experiment this was found to occur. In order to increase

1 Wied. Ann., 64, 233; 1898. 
the effect the parallel wires were replaced by an induction coil as shown in Fig. 51. $M$ is the microphone which is placed in series with a cell and with $P$, the primary of the induction coil. The secondary of this coil is placed in series with the arc.

Braun, in the same year, ${ }^{1}$ stated that the larger the current flowing through the arc, the greater the change in the amount of energy of the arc, when a small E.M.F. is in-

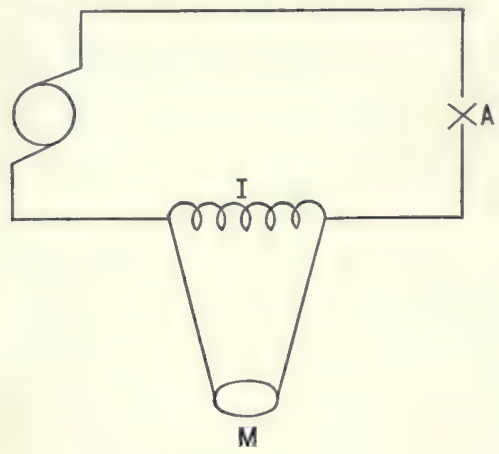

FIG. 52 .

duced in the arc circuit. This would be true if the current in the arc obeyed Ohm's law, but since it does not, we can not find from theory the best conditions for large changes in the energy of the arc.

Several methods have been used in connecting the microphone in the circuit. ${ }^{2}$ A very common one is that shown in Fig. 52, where the microphone $M$ is in shunt with an inductive resistance $I$, so that each change in the resistance

1 Wied. Ann., 65, 358; 1898.

2 Ruhmer, Elektrot. ZS., 22, I97; I9OI, and 23, 859; 1902.

Simon, Elektrot, ZS., 22, 5 I0; I90I.

Duddell, Lond. Flec., 46, 269; 1900. 
of the microphone causes a change in the current through the arc.

In making a receiver for this kind of wireless telephone use was made of two facts; first, that with each change of the current through the arc there is a change in the intensity of the light of the arc; and second, that the electrical resistance of selenium changes whenever the intensity of the light falling on it changes, so that selenium may be used to detect changes in the current through the arc. This is done by making the selenium one of the arms of a Wheatstone bridge and putting a telephone receiver in the place of the galvanometer. By using an unusually sensitive piece of selenium Ruhmer was able by such an arrangement to send messages seven kilometers.

However, this method has not been found to be a commercial success. In the first place it can be used only when the light of the arc shines on the receiving apparatus. A fog, for example, would destroy its usefulness. Again there can be no building or elevation obstructing the view between the transmitter and the receiving apparatus. Moreover the system can not be used in the daytime, for changes in the intensity of the light due to any change in the light of the arc are very small as compared with ordinary daylight, and finally under the most favorable conditions the sound received is but a poor imitation of that spoken.

Whistling Arc. - The second device for wireless telephony has been somewhat more successful. It depends on a discovery which appears to have been first made by E. Thomson in $1892^{1}$ and later in a slightly modified form by Duddell. ${ }^{2}$ The principle of the discovery was the same

1 U. S. Patent No. 500,630.

${ }^{2}$ Lond. Elec., 46, 310; I900. Inst. Elec. Eng., 30, 232; 1900. 
in both cases, i.e., that when a condenser with self-induction in series with it is shunted about an arc, oscillations may be produced in the arc. The connections are shown in Fig. 53 where $A$ is the arc, $C$ the condenser, $I_{2}$ the inductance in series with condenser, and $K$ a key for opening or closing this circuit. In general for the production of the oscillations it is necessary to have an arc of high resistance and a shunt circuit with low resistance. For example,

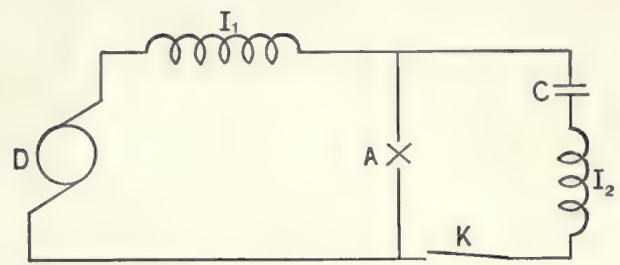

FIG. 53 .

solid carbons must be used instead of cored carbons. The nearer the arc is to extinction without being extinguished the more apt it is to give the oscillations. With the apparatus as shown by Duddell it was necessary to have less than 2 ohms in the shunt circuit with about 5 millihenrys inductance and a capacity of from I to 5 microfarads.

This is due to the fact that when the current through the arc increases, the potential difference needed to produce it decreases. Thus, if the key at $K$, Fig. 53 , is closed while the current is flowing through the arc, the current will rush into the condenser. If there is also inductance at $I_{1}$, the current flowing from the battery will not have time to change more than a slight amount during the period that the condenser is being charged. Under these circumstances the current which rushes into $C$ will be taken 
from that which had previously been flowing through the arc.

A larger potential difference will be needed to force this smaller current through the arc, as may be seen from Fig. 54 which gives diagrammatically the relation between

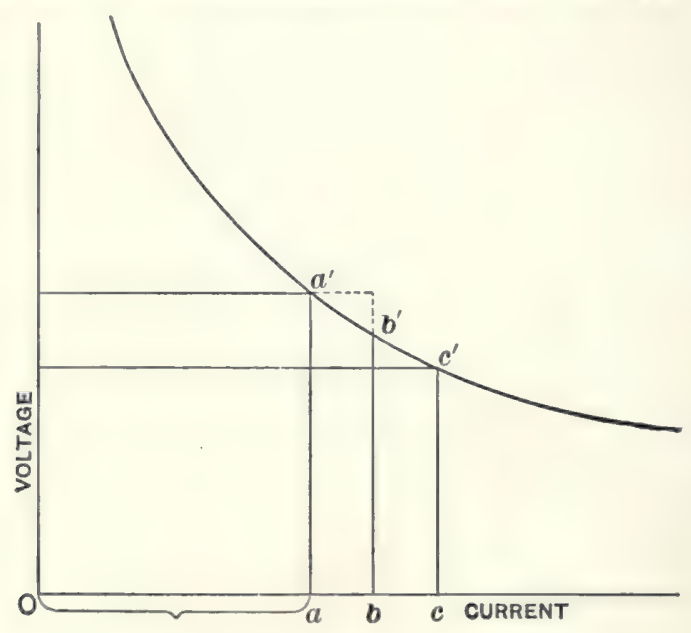

Fig. 54 .

the potential difference and the current of the arc. Thus let $O b$ represent the sum of the currents flowing to the arc and the condenser. Let $a b$ represent the current flowing into the condenser at some given moment. $O a$ will then be the current flowing through the arc. $\quad b b^{\prime}$ is the potential difference at the terminals of the arc when no current is flowing to the condenser, and the larger quantity, $a a^{\prime}$, is the potential difference when part of this current is flowing to the condenser.

There will, however, be a limit to the increase in the voltage which can be supplied to the arc and when this is 
reached, the condenser will cease taking current from the arc, the arc will require less voltage, and the condenser will begin to discharge through it. This added current will cause the voltage of the arc to drop still more until it reaches the value shown by the line $c c^{\prime}$. Eventually a limit will be reached in this direction also. As the current from the condenser ceases to flow through the arc, its voltage begins again to increase and the process will be repeated.

Energy is used in the condenser circuit both to produce the heat due to the resistance of the circuit and to radiate electrical waves. This energy is given to the condenser circuit, because the potential difference is higher when the current is flowing into the condenser than it is when it is flowing out, so that more energy is delivered to the circuit than is received from it. The steeper the characteristic curve of the arc the more the energy that will be delivered to the condenser circuit. ${ }^{1}$ It has also been shown by Simon ${ }^{2}$ that the greater the hysteresis of the "dynamical" characteristic curves, the greater will be the amount of energy delivered to the condenser.

In general, arcs having steep characteristics are those having terminals which cool off quickly, or those whose resistance is in some way artificially increased. Thus it has been found that an arc between copper terminals can be used for producing oscillations to better advantage than one between carbons and that deflecting the arc with a magnet is advantageous. ${ }^{3}$ Increasing the pressure of the

1 Granqvist, Mitt. d. königl. Ges. D. Wiss. zu Upsala, (4), I, 62; I906. Beib., 3I, 840; 1906.

2 Phys. ZS., 4, 737; 1903, 6, 297; 1905, and 7, 433; 1906.

Elektrot. ZS., 26, 818 and 839; 1905, and 28, 295; 1907.

Jahrbuch der drahtlesen Telegraph. und Teleph., I, I6; 1907.

a Poulsen, Elektrot. ZS., 27, 1040; 1906. 
surrounding gas is helpful, and surrounding the arc with steam, coal gas or hydrogen gives better results. ${ }^{1}$

The time that is taken for charging and discharging the condenser depends on the capacity and inductance of the condenser circuit. The period of the swing would equal $2 \pi \sqrt{L C}$, if the resistance of the arc were constant, and it was indeed suggested by Janet ${ }^{2}$ that use could be made of this fact to determine the coefficient of inductance of any circuit placed in series with the condenser. However, the very existence of the phenomenon depends on the fact that the resistance of the arc is not constant and it has been shown that the period of oscillation depends not only on the capacity and inductance, but also on several other of the conditions under which the arc is working, so that this method can not be used in determining inductance. ${ }^{3}$

The charging and discharging of the condenser produce electric waves and it is possible to make use of these in wireless telephony, but in order to send a sufficient amount of energy out into space, it is necessary to have high frequency. Many arcs which show steep characteristic curves for slow changes do not have steep curves when the changes are very rapid, as has been shown by Duddell for carbon arcs. If, therefore, oscillations are to be produced, it is

1 A very complete review of this subject showing oscillographs of several different arcs has been given by Blondel. (Ecl. Elec., 44, 4I and 8I; I905.)

2 C. R., 134, 462 and 821; 1902.

${ }^{3}$ Ascoli and Manzetti, Rend. Acc. Linc., (5), II, 2 sem. II; IgO2.

Corbino, Sc. Ab., 7, 537; 1903.

Duddell, Lond. Elec., I, 902; I903.

Maisel, Phys. ZS., 4, 532; 1903.

Salomonson, Sc. Ab., 6, 167; 1903; Ecl. Elec., 34, 202; 1903.

Granqvist, Boltzmann Festschrift, 799; 1904.

Heinke, Ver. d. Ver. z. Beford. d. Gewerbfleisses, 83, 403; r904.

Nasmith, Phys. Rev., 27, 1I7; I908, and 32, 69; IgIr. 
necessary to use arcs which not only have steep characteristic curves, but also have such curves for rapid oscillations.

Among the different forms of arc which have been used successfully is that with graphite terminals. Austin ${ }^{1}$ has. given the following data concerning such an arc.

Electrodes

Diameter .

Resistance in series with arc

Arc current

Voltage across arc

Voltage open circuit

Arc length . .

Inductance in shunt circuit. ............

Capacity

Alternating current in shunt. graphite, ends flat. I $2 \mathrm{~mm}$.

about $50 \mathrm{ohms}$.

4 amp. 26 volts. 242 volts. about $0.3 \mathrm{~mm}$. 0.009 millihenry. 0.4 micro-farad. 4 amp.

He found still better results with the arc in a stream of hydrogen or steam. But the best results which he obtained were with the arc between silver terminals in air under a pressure of about 7 atmospheres and with an E.M.F. of 4500 volts. The arc then takes on all the characteristics of a very rapid spark discharge and it is possible to develop a large amount of power in the shunt circuit.

\section{Applications of the "Whistling" Arc to Wireless Te-} lephony. - The application of the whistling arc to wireless telephony is a subject belonging to works on that subject and can not be adequately treated here. However, two diagrams are given which show how the arc is connected to the other parts of the transmitting apparatus, so as to send electric waves out into the ether. In order to do this two additions must be made to the device shown in Fig. 52 . First it must be changed so that modifications may be made in the electric oscillations which shall correspond to the

1 Bull. Bureau Standards, 3, 325; I907. 
vibrations of the voice. One of the simplest arrangements for this purpose is shown in Fig. 55. $A$ is an arc producing

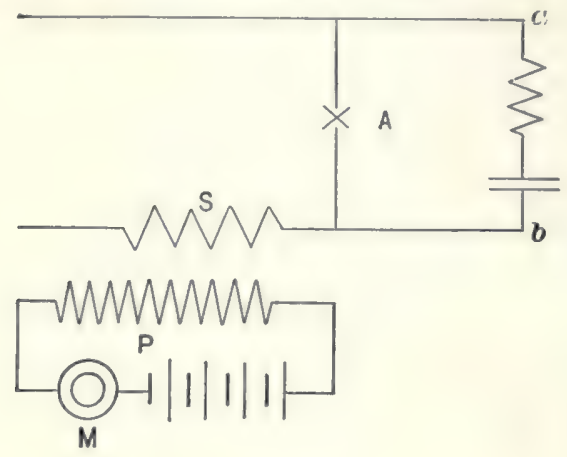

FIG. 55.

oscillations in the circuit $a b . \quad M$ is a microphone producing disturbances in the secondary circuit. $P$ is the primary of an induction coil which is in series with $M . S$ is the secondary circuit of the coil and is in series with the arc.

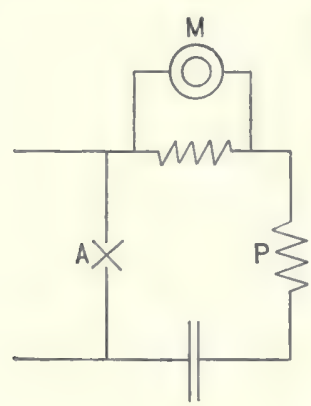

FIG. 56 .

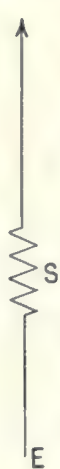

The disturbances in $P$ induce currents in $S$. These currents modify the conditions in the arc and in $a b$.

The oscillations produced by $S$ are very much slower than those produced by the arc, so that there will be some groups of waves sent out from $a b$ which are somewhat more intense than other

E groups. The variations in these groups will correspond to the vibrations produced in the microphone $M$.

A second manner of connecting the microphone is shown 
in Fig. 56, where the microphone is placed in shunt with the inductance. Changes in the resistance of the microphone will again produce changes in the intensity of the oscillations about the arc.

A further addition must be made to the apparatus in order that the oscillations produced by the arc may be radiated into space and to do this effectively it is necessary to have a higher E.M.F. than that which would be given by the device already described. For this purpose a stepup transformer is introduced into the circuit in shunt with the arc as shown in Fig. $5^{6}$, where $P$ is the primary of the transformer and $S$ is the secondary. One end of the secondary is connected to the earth and the other to an aërial wire. ${ }^{1}$

1 Partial list of articles published on the second method of wireless telephony which I have not otherwise mentioned:

Nussbaumer, Phys. ZS., 5, 796; r904.

Ruhmer, Elektrot. ZS., 27, 1060; 1906.

Rozanski, Beib., 31, 845; 1907.

Brown, Lond. Elec., 58, 20I; 1907.

Vreeland, Lond. Elec., 58, 685; 1907 .

Blondel, Lond. Elec., 6o, 216; 1907.

Fessenden, Lond. Elec., 59, 987; 1907.

Mosler, Elektrot. ZS., 28, I42; 1907.

Fleming, Lond. Elec., 59, 9I4; I907.

Sahulka, Elektrot. ZS., 28, 1063; 1907.

Traubenburg, Elektrot. ZS., 28, 559; 1907.

Eisenstein, Elektrot. ZS., 28, 830; 1907 .

Schapira and Loewe, Lond. Elec., Eng., Nov. 7; $190 \%$

Cartelli, Lond. Elec., 62, 609; 1909. 


\section{CHAPTER IX.}

\section{Theory of the Electric Arc.}

UNTIL the ionic theory of discharge through gases was developed, there was no explanation of the phenomena of the electric arc which was of value. But in recent years several explanations based on this theory have been suggested and an outline of these will be given in the following pages. The first attempt in this direction was made by the present writer in $1900 .{ }^{1}$ A few years later a more complete explanation was given by Stark, ${ }^{2}$ and a slightly different one by Sir. J. J. Thomson. ${ }^{3}$

All of these explanations assume that the current is carried by ions and a discussion of them will require us to understand, first, what is meant by ions; second, how they are produced; and third, their effect on the potential gradient between two electrodes, especially in a place where ions of one kind only exist.

Definition of Ions and Electrons. - Certain causes appear to break atoms into parts, one part of each atom being charged positively and one part negatively. These parts have been called ions. They tend to attach themselves to uncharged atoms, and these clusters of atoms are also called ions, so that an ion may be defined as an atom, a part of an atom or a cluster of atoms which has a positive

1 Phys. Rev., I0, I5I; 1900.

2 Ann. d. Phys., 12, 673; 1903.

3 Conduction of Electricity through Gases, Ist. Ed., p. 4I6. 
or negative charge. From the derivation of the word, it signifies moving, and the name is applied to nearly anything that will move in an electric field and is too small to have any other name.

This definition includes ions in liquid solutions, but in this discussion we shall consider only the ones which are found in gases. These have different properties from those of electrolytes and the phenomena of the arc are not the same as those existing when a current of electricity passes through a liquid. These differences can be better understood after the facts relating to the arc have been more fully discussed.

The movement of the ions constitutes a current of electricity. Ions thus render the space where they exist conducting, and by this means their presence is ordinarily detected.

The negative part which is broken off from an atom or molecule is much smaller than the remaining positive part. This ion which is a small fraction of an atom is often called an electron. This very quickly becomes attached to an atom or molecule, and though it is still an ion, it is no longer called an electron. An electron, then, is a negative ion which has a mass very much smaller than that of an atom. In some cases of discharge through gases nearly all of the negative ions are electrons. Cathode rays, for example, are streams of such parts of atoms. In other cases the electrons so quickly combine with molecules that their presence can scarcely be detected, as when discharge in air at atmospheric pressure is produced by Röntgen rays. In discharge through hot flames there are probably present in large numbers both electrons and negative ions of atomic size. 
Causes Producing Ions. - Ions thus formed do not continue indefinitely. They quickly recombine and again form uncharged atoms. Thus, to have a continuous supply of ions, there must be a continuous production. Among the causes producing them may be mentioned radio-active substances, Röntgen rays, ultra-violet light, the impact of ions on atoms, chemical action, especially at high temperatures, and incandescent solids. Of these, two only will be of interest to us at this time, namely, the impact of ions on atoms, and incandescent solids.

Ionization by Impact. - Let us first consider ionization by impact. If ions exist where there is an electric field they tend to move with an accelerated velocity. Under ordinary conditions, they can move but a short distance before colliding with an atom or molecule of gas. If the atom or molecule is hit with sufficient velocity, it is broken into parts which are charged and which are indeed new ions. Thus, cathode rays and the electrons produced by ultraviolet light or incandescent solids ionize the gas through which they pass and render it conducting. It has been shown by Townsend that positive ions will produce the same effect. ${ }^{1}$

The velocity of an ion at any instant depends on the force which has been acting on it and upon the distance covered since its last collision, or in other words upon the potential difference through which it has passed since it was at rest. Consequently, to have sufficient velocity to ionize by impact, an ion must pass through a certain potential difference. If the mean free path is too short, or the electric force too small, no effect will be produced. The measurements made by Townsend indicate that the 1 Phil. Mag., (6), 6, 598; 1903. 
negative ion must pass through 25 volts in order to ionize in air, and the positive ion must pass through 70 volts. ${ }^{1}$ Davis ${ }^{2}$ gives II.7 volts as the value needed to ionize with the negative ions.

The ions can also ionize when they hit the molecules of a solid, as when the negative ions hit the surface of the anode, or the positive ones hit that of the cathode. The potential difference needed in this case is greater than that needed with the molecules of a gas. Thus when the positive ions hit a cold cathode, they need to pass through at least 300 volts in order to produce electrons. The potential difference needed by the negative ions is not so well known, but it is probably about the same as the anode drop of the unstriated discharge in a vacuum tube which is approximately 20 volts. ${ }^{3}$

Ionization by Hot Solids. - It has long been known that substances at a high temperature discharge electrified bodies, and in recent years it has been shown that the rate of such discharge may be very large. This was shown by Richardson in $1903^{4}$ when a hot wire which was charged negatively was placed in a vacuum. A little later Wehnelt ${ }^{5}$ found that the discharge from some of the oxides, as, for example, calcium oxide, was very much greater than that from a metal.

It was believed by Richardson that this discharge was caused by electrons coming from within the metal and passing through its surface because of the great velocity which they have at high temperatures, and he derived the

\footnotetext{
1 Phil. Mag., (6), 5, 395, and (6), 6, 613; 1903 .

2 Phys. Rev., 24, 102; 1907.

3 Wied. Ann., 68, 752; I899.

4 Phil. Trans., 201 A, 497; 1903.

- Ann. d. Phys., 14, 439; I904.
} 
formula $I=A e^{\ominus}$, where $I$ is the current passing from the metal, $\theta$ the absolute temperature, and $A$ and $Q$ are constants, depending on the kind of metal used. There are somewhat serious objections to the assumptions made by Richardson in deriving this formula, ${ }^{1}$ but the formula itself has been found to be approximately true for several of the metals and will give an idea of the rapidity with which the current increases when the temperature is raised.

Fall of Potential through the Arc. - To pass then to the arc, we have found that it may be divided into three

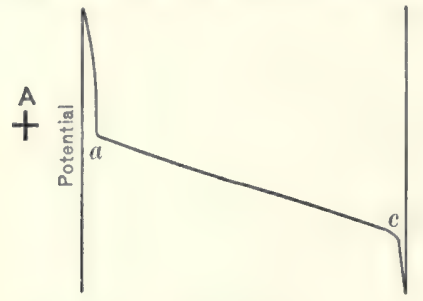

FIG. 57 . parts, the part near the anode, $A$, Fig. 57: at which

$\underline{\mathrm{c}}$ there is a sudden drop in potential, as is shown in the diagram; that through the arc, where the fall in potential is gradual and nearly uniform, as $a c$; and that very near the cathode, $C$, at which the drop is again sudden.

To understand these peculiarities we may begin with the case where ionization is produced in the gas between the two electrodes, but not in their immediate neighborhood; we may next consider the phenomena introduced by the impact of the ions on the electrodes, and finally may pass to the case where the electrodes are incandescent.

An example of the first will be found when two plates are placed on opposite sides of a flame and at some distance from it. For example, let $a c$, in Fig. 58 , be a space where ionization occurs. This space will then contain both posi-

1 Wilson, Phil. Trans., 202 A, 243; I904.

Horton, Phil. Trans., 207 A, I63; I908. 
tive and negative ions. The space $A a$ will contain only negative ions and $C c$ only positive ions. Where there are ions of one sign only, the potential gradient changes rapidly and the drop in potential in the immediate neighborhood of $A$ and $C$ may become very large. The mathematical treatment of this proceeds

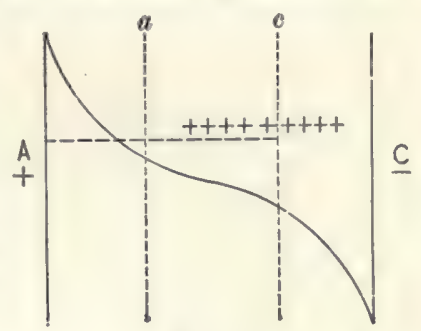

FIG. 58. easily in simple cases from the equation

$$
\frac{d^{2} V}{d x^{2}}+\frac{d^{2} V}{d y^{2}}+\frac{d^{2} V}{d z^{2}}=-4 \pi \rho
$$

For example, when the lines at $A, a, c, C$ represent infinite planes, $V^{2}>\frac{32 \times 10^{11} \pi i b^{3}}{k}$, where $b$ is the distance $A a, V$ the change in potential through $A a, k$ the velocity of the ions per unit electric force and $i$ the current per unit cross section. The units used are volts, amperes, centimeters and seconds. ${ }^{1}$ Thus if $i$ equals $10^{-8}$ amperes, $b$ equals $I \mathrm{~cm}$., and $k$ equals Io, then $V$ is greater than roo volts.

Ionization at Surface of Cathode, with Discharge at Low Pressure. - This discussion applies also to the case where ionization is produced by impact, as with discharge through a vacuum tube. But here a complication is introduced. If no electrons come from $C$, all those between the plates will soon pass to $A$. Let us suppose that the region $C c$ has thus been cleared of negative ions. There will then be a large drop in potential here, since the positive ions will have moved into this region and there is always a large

1 Phys. Rev., 12, 79; I90I. 
drop in potential in a region where there is only one kind of ions. If this drop is sufficiently large, the positive ions will attain high velocities, and by impact on the boundary surface at $C$ they also will ionize. We shall then have a two-sided action. The negative ions coming from $C$ will ionize in the space $a c$ and the positive ions from $a c$ will ionize at $C$.

As the negative ions thus formed begin to fill the space $C c$, the drop in potential will become smaller. If, however, it becomes small enough, ionization at $C$ will cease and the potential difference will again become greater. For a condition of equilibrium the potential difference must be large enough so that positive ions will ionize at $C$.

Ionization at Surface of Anode. - We find that the phenomena at the anode are similar to those at the cathode. The essential difference is that the negative ions ionize by impact much more easily than the positive. The drop in potential at the anode is consequently less than that at the cathode. If no ions came from the anode, there would be a large drop in potential near it, as in the corresponding case with the cathode. This would cause the negative ions to move with great velocity and their bombardment of the surface layer at $A$ would produce new ions. As before, a condition of equilibrium exists when the drop in potential is sufficiently large to cause many of the negative ions to produce new ions at the boundary surface.

Ionization at Cathode of Arc. - The arc is in many ways similar to the discharge in a vacuum tube. There is a drop in potential at the anode, a gradual fall through the gas and another sudden drop at the cathode. The essential differences between the two phenomena are that in the arc the drop in potential at the cathode is much smaller than it is 
in a Crookes' tube and the cathode itself must be very hot. In the arc the cathode drop instead of being 300 volts may be as low as 5 volts (p. 96). That the cathode must be hot was shown by Luggin ${ }^{1}$ who found that a carbon disk kept cool by being rotated could be used as the anode, but not as the cathode of the arc. Stark and Cassuto ${ }^{2}$ showed the same thing for a metal kept cool by being rotated, and for an electrolyte which can never be raised to a high temperature. In some work by the present writer ${ }^{3}$ it was found that the anode end of an arc in a vacuum could easily be made to jump to a carbon electrode placed near the arc, but that the cathode end could not be made to do so, except when the pencil was white hot.

\section{Assumption that the Electrons are from Within the} Cathode. - As a result of this fact all agree in assuming that the production of the ions at the cathode is the basis of any explanation of the arc. They differ, however, as to how these ions are produced. According to one view the cathode is heated by the impact on it of positive ions, until it becomes sufficiently hot to send out ions from within the solid to the surrounding gas, as in experiments performed with hot platinum wires. According to the other view the electrons are produced by the impact of the positive ions on the molecules at the surface of the cathode, and the cause for the small cathode drop is the fact that ionization occurs very much more easily when the surface of the cathode is at a high temperature. Both Stark and Thomson have made the former assumption the basis of their explanations. They suppose the hot cathode to be the origin of the negative ions and that the rise in potential

1 Wien. Sitzungber., 96, 2 A, 767; 1887 .

2 Phys. ZS., 5, 264; 1904.

3 Phys. Rev., 19, II9; I904. 
need be only great enough, so that the positive ions by their bombardment of the cathode should raise it to the necessary temperature.

There are, however, objections to this view. The most important of these is that there are many substances which can be used as the cathode of an arc and yet melt and vaporize before a temperature is reached at which negative ions are given off. The most noteworthy example of this occurs with the arc between mercury terminals. Mercury vaporizes, especially in a vacuum, at temperatures far below that at which negative ions pass from a metal.

In reply to this last objection it was shown by Stark ${ }^{1}$ that even in the case of mercury there is a point at the cathode which is very hot and at this point there is a continuous spectrum. This fact was also pointed out by Arons. ${ }^{2}$ Apparently the rise in temperature occurs so suddenly that the mercury does not become a gas until after the atoms are set in such violent vibration that they give out light.

If we should assume that this difficulty may be thus explained, there is still the fact that in a vacuum the metals which melt the most easily can be made the cathode of the arc, while those, like iron and copper, which do not melt easily can not be so used. We should expect that iron would give off ions at least as readily as mercury, if the phenomenon depended only on raising the metal to a high temperature.

There are also certain minor difficulties met with in this explanation. For example, the measurements made by Richardson on the discharge from hot carbon ${ }^{3}$ would indi-

1 Physik. ZS., 5, 750; 1904.

3 Phil. Trans., 20r A, 497; 1903. 
cate that at $3140^{\circ} \mathrm{C}$, which is the temperature of the cathode according to Reich, ${ }^{1}$ the rate of discharge from the cathode should be $2 \times 10^{8}$ amperes per sq. cm., whereas the measurements which Reich made on the size of the cathode spot indicate that the current is but 318 amperes per sq. $\mathrm{cm}$.

Second Explanation of Ionization at Cathode. - According to a second explanation the ionization at the cathode is produced by the impact of the positive ions on its surface. In the discharge from a cold cathode such ionization is supposed to occur and the only objection to assuming that it occurs at the cathode of the arc is that the cathode drop is here much smaller. However, it has been shown by Hittorf $^{2}$ and Cunningham ${ }^{3}$ that the cathode drop becomes smaller as the temperature of the cathode is raised. The same occurrence has been found when using hot calcium oxide for the cathode. ${ }^{4}$ It would in fact be surprising if it were not much easier to ionize molecules which are at the temperature of the arc, since even without impact they emit some electrons.

Almost conclusive evidence in favor of this view is given by the similarity between the glow discharge in a partial vacuum, the luminous discharge from hot calcium oxide, and the arc, since with the first two of these we certainly have ionization at the cathode produced by impact of positive ions. The similarity between these is shown by the fact that it is quite possible to pass by gradual changes from the ordinary form of discharge in a vacuum, where the cathode drop must be several hundred volts, through the discharge from a heated cathode, where Cunningham

1 Phys. ZS., 7, 73; 1906.

2 Wied. Ann., 21, I33; I884.
3 Phil. Mag., (6), 9, 202; 1905.

4 Phys. Rev., 32, 507; I9II. 
found it to be 250 volts, to that from hot lime, where it varies from 70 to 12 volts, and then to the arc, where it may be as low as 6 volts. The luminous discharge from hot calcium oxide is identical with the arc in a vacuum, except that with the arc no independent source of energy is needed to heat the cathode ${ }^{1}$ and this is a thing which has nothing to do with the manner in which the ions are produced.

That there is ionization by impact at the cathode with the luminous discharge from hot calcium oxide is shown by the following. With hot calcium oxide it is possible to have a very small non-luminous discharge and with the same current heating the cathode and the same potential difference between the electrodes to have a large luminous discharge, providing the discharge has momentarily been raised to an amount sufficiently high to start the larger discharge. The number of electrons emitted from within the calcium oxide can not be greatly different in the two cases, for the temperature of the cathode has not appreciably changed, and a study of the potential between electrodes shows that the increase in current can not be due to a movement of the positive ions toward the cathode. The only way to explain the increase is by assuming that in the second form of discharge a greater number of electrons is produced at the cathode by the impact of a greater number of positive ions. The appearance of the rays streaming from the cathode confirms this view. If then we have ionization by impact with hot calcium oxide, we undoubtedly have the same manner of ionization in the arc.

\section{Causes Determining the Amount of the Cathode Drop. -} The immediate cause of the drop in potential at the cathode ${ }^{1}$ Phys. Rev., 19 , 36r; rgo9. 
is the accumulation in its neighborhood of positive ions which have been drawn from the vapor of the arc but have not yet reached the cathode. This accumulation can not become indefinitely great, for, when it increases, the current flowing from the cathode increases, and this soon causes the drop in potential through the remainder of the circuit to become as great as it is possible to have it with the given E.M.F. On the other hand the cathode drop can not fall below a certain value, for it must be great enough both to cause negative ions to be formed at the cathode and to cause these to produce positive ions by their impact on the molecules of the gas. Whichever of these is the greater will determine how great the cathode drop must be.

One can not at present say with certainty which of these is the greater. The cathode drop in some cases is not only much smaller than that needed to produce electrons by the impact of the positive ions on a cold cathode, but also much smaller than that needed to produce positive ions by the impact of electrons on the molecules of a gas. ${ }^{1}$ Apparently at high temperatures both forms of ionization occur much more easily than at low. Since we do not know the exact relation between the temperature and the potential difference needed for ionization with either the positive or negative ions, we can not state definitely which is the lower at high temperatures.

There are, however, reasons for believing that in all cases the potential difference needed for ionization by the positive ions is the greater. We shall find, for example, when we examine the drop in potential through the gas of the arc, that in some way the ionization of the arc is kept up, though the mean free path of the electrons in some cases

${ }^{1}$ Phys. Rev., 24, 93; 1907. 
is through not more than 0.03 volt. If ionization by the electrons coming from the cathode can be produced by anything like this potential difference, then the drop which we actually find at the cathode must be determined by the potential difference needed for ionization with the positive ions and not by that needed for the negative ones.

Ionization at the Anode. - There are two distinct sets of conditions to be found at the anode, depending on whether the cathode rays hit directly on the anode or not. The first conditions exist only when the arc is in a very high vacuum. The negative ions then go directly from the cathode to the anode. There is then no luminosity between the electrodes which is at all comparable with that ordinarily existing in the arc and there is apparently but very slight ionization of the gas between the electrodes.

In all probability there is then no ionization at the surface of the anode. Apparently the conditions are identical with those in a vacuum tube with currents such as were studied by Skinner ${ }^{1}$ and by myself. ${ }^{2}$ In these investigations it was found that the anode drop varied from one or two volts to over one hundred volts. It apparently depended on the average velocity of the negative ions when they reached the anode, the anode drop decreasing as the velocity of the ions increased. This is what would be expected if the current near the anode is carried by ions of one sign only as is shown on page $\mathrm{I} 6 \mathrm{r}$.

Whether this is the whole explanation of the anode drop with the arc in a vacuum under very low pressure is, no doubt, open to question. The fact that Stark, Retschinsky, and Schaposchnikoff ${ }^{3}$ found that it was nearly independent

1 Phil. Mag., (6), 2, 637; 190r. ${ }^{2}$ Phys. Rev., 29, 351; 1909.

3 Ann. d. Phys., 18, 244; I905. 
of the current under these conditions would indicate that it is not, but it is doubtful whether any more complete explanation can be given until some method is found for determining the velocity of the negative ions when in the immediate neighborhood of the anode.

Ordinarily the ions are stopped before reaching the anode and we then have the second set of conditions; namely, those which occur when there is a large amount of ionization and of luminosity between the electrodes. Under these conditions there is ionization at the anode. If there were not, the drop would be very much larger than it is.

The amount of the anode drop under such conditions appears to be determined by the potential difference necessary to produce ionization at the surface of the anode. That is, the drop increases until it becomes sufficiently large to cause the negative ions by their impact to disrupt the atoms at the surface of the anode. Of these new atoms the positive ones move toward the cathode and by their electrostatic influence keep the potential difference from becoming any larger.

The phenomenon is much the same at the anode as at the cathode, ionization at the anode being produced by the impact of the negative ions, and that at the cathode by the impact of the positive ones. It is not necessary, however, for the temperature of the anode to be high, while it is necessary that the cathode should be very hot. Moreover at the cathode there is a production of negative ions at the surface and of positive ones at a short distance from it, while the positive ions produced at the anode play no part in the production of negative ones.

This view is in harmony with the fact that the anode drop is nearly independent of the amount of the current. 
The same reasoning that was given in connection with the cathode drop would apply here. Whether the number of negative ions going to the anode is large or small, a new ion must be formed for each negative ion coming to the anode, and a definite potential difference is needed for this purpose.

The view was expressed by Sir. J. J. Thomson ${ }^{1}$ that the ionization at the anode is caused by the anode being heated to a high temperature and that the anode drop is determined by the amount of energy necessary to keep the anode at this temperature. However, there are many forms of the arc where the anode is not raised to a temperature which can be considered high, so that the anode drop would not appear to be determined by such a requirement.

Blondel $^{2}$ expresses the view that when the anode is vaporized it gives out positive ions. I have not been able to find anything that confirms this view and the fact that the anode drop in a mercury arc is much the same with a mercury anode which is easily vaporized as it is with an iron anode which is not vaporized would seem to make this view untenable.

Discharge of Ions to Electrodes. - We have considered the manner of production of ions at the electrodes, but it is also true that the ions coming to them must in some way lose their charge. Whether the charge is detached from the ion and given to the electrode or is neutralized by some charge coming from the electrode, we do not know, but in either case it is hardly to be expected that the action will take place without loss or gain of energy. That it does

1 "Conduction of Electricity through Gases," Ist ed., p. 424.

2 Trans. Inter. Elec. Cong., St. Louis, 2, 747, 1904. 
not happen without complicating action when cathode rays strike an anti-cathode is evident, for we there have secondary rays given off and also a large development of heat. As far as the writer is aware there has been no work, either experimental or theoretical, done on this subject in the case of the arc.

Ionization of the Gas between Electrodes. - With very low pressures the current is carried almost entirely by electrons which appear as cathode rays streaming out toward the anode. These, no doubt, ionize the gas through which they pass. The ability to ionize the gas is one of the well-known properties of such rays and certainly ionization of the gas must be produced in some way, since positive ions come from the gas and heat the cathode.

As the pressure of the gas or as the distance between the electrodes is increased, a glow appears at the anode. Under some conditions striations appear as was shown in Fig. 20. Ordinarily with increase of pressure there is a continuous glow between the electrodes. Increasing the pressure further does not change the character of this glow, except to make it more intense.

With this form of the arc it is also certain that there is ionization between the electrodes. In air, for example, the electrons coming from the cathode can not go far before being stopped by collision with the molecules of the air and recombination between the ions must take place in many cases. If so, there must also be fresh ionization to keep up the supply of ions.

Moreover we know that positive ions are produced in the gas, since they are continually moving toward the cathode, as is shown by the potential gradient between the electrodes. This gradient has been found in all cases 
to be practically uniform, which could not be, if there were not an equal number of positive and negative ions through the gas.

This ionization is no doubt produced by the impact of electrons on the atoms or molecules. It would seem at first as if there were other possible explanations for the ionization. It has been shown, for example, that ultraviolet light ionizes gases. ${ }^{1}$ Light of the short wave length needed to produce such ionization is not known to exist in any of the forms of the arc, but since it is completely absorbed by a thin layer of air, it is quite possible that it has been overlooked and does indeed exist in all forms of the arc.

Such light may indeed be an aid in producing ionization, but it can not be the only source, for it comes only from vibrating ions, and its energy comes from the energy given to a system of ions when the molecule is broken into two parts. The energy of vibration of one pair of ions will not be sufficient to set another pair into similar vibration of equal intensity, for a large amount of this energy is being continually radiated away from the arc and another large part is changed into heat and conducted away, and it would require all of the energy of one vibration to produce another like it. The number of vibrating atoms will, therefore, diminish, or some other source of energy must be changed into that of vibration. In other words, if the number of vibrating ions is to be kept constant the electrical energy must in some way be changed into that of ultra-violet light, and the only known way for this to be done in a gas is by impact of the ions on the molecules.

The same kind of reasoning will show that it is not 1 Phys. Rev., 32, I; I9II. 
possible for any other kind of radiation, such as Röntgen rays, or "entladungstrahlen" to be the sole source of the ionization.

On the other hand there are serious objections to believing that impact of ions is the sole cause of ionization. The first of these is that the mean free path of the electrons is through too small a difference of potential to produce ionization. The work of Davis ${ }^{1}$ indicates that at ordinary temperatures the ions must move through II.7 volts in order to ionize. We may get some idea of the potential difference through which the electrons in the arc pass by assuming that the mean free path of the ions varies inversely as the density of the gas, that the electric force of the arc is 27 volts per centimeter, ${ }^{2}$ that the mean free path of the electrons in air at a pressure of I $\mathrm{mm}$. and a temperature of $20^{\circ} \mathrm{C}$. is $0.0475 \mathrm{~cm}$. as found by Townsend, ${ }^{3}$ and that the temperature of the gas of the arc is $3700^{\circ} \mathrm{C}$., the temperature of the anode as given by Waidner and Burgess. ${ }^{4}$ The mean free path of the electrons computed from these assumptions is through a potential difference of 0.025 volt, which is quite different from II.7 volts, the required amount.

In the second place the electric force through the arc does not increase in inverse proportion to the length of the mean free path of the ions, as it should, if the ions are caused by the impact. Thus, in experiments performed by myself, it was found that the electric force in the arc between two solid carbons was 23.4 volts per centimeter when the pressure was $730 \mathrm{~mm}$., and $\mathrm{I} 3.2$ volts with a pressure of $2 \mathrm{~mm}$. The temperature had apparently

1 Phys. Rev., 24, 93; 1907.

2 Mrs. Ayrton's "Electric Arc," p. 23r.
3 Phil. Mag., (6), I, 2 I5; I9or.

4 Phys. Rev., 19, 250 ; I904. 
changed but little, so that the mean free path in this last case was approximately three hundred times greater than that in the first, while the electric force was only one-half as great. Again, in the data given by Knipp, the change in the electric force was much smaller than that in the pressure.

It has been suggested by the author that these difficulties can be easily explained if we assume that the molecules of a gas are much more easily ionized when the temperature of the gas is high than when low. ${ }^{1}$ We have already seen that the molecules on the surface of a solid are more easily broken apart if the solid is very hot and in all probability the same thing occurs with gases. This explanation seems the more reasonable since the gas in the arc is always at a high temperature. Even when the pressure of the gas was only $0.5 \mathrm{~mm}$., the temperature of the arc between carbons was sufficiently high to meit platinum. As has been shown there is a difference of opinion regarding the mercury arc, but it seems safe to assume that its temperature is at least high.

Such an assumption will very easily explain the difficulties which have been mentioned. It would, for example, explain the fact that the larger the current flowing through the arc the less the electric force required to produce it, for when the current increases there is an increase in the heat developed in the arc, and if ionization occurs more easily at high temperatures, then the electric force needed to produce the greater amount of ionization may remain nearly constant or even become smaller.

It would explain the phenomena at different pressures. When the mean free path of the ions is sufficiently large, 
ionization will be produced without high temperature and we then have the arc at lower temperatures. As the mean free path becomes less, the average velocity of the ions becomes less, and in order to have ionization, the temperature must be raised. At still higher pressures the mean velocity of the ions becomes very small and the temperature is the determining factor. Then it makes little difference what the mean free path of the ions may be, if only the temperature is sufficiently high.

This view is also in harmony with the facts observed by Pollak. The mercury vapor above the arc was found by him to be but slightly conducting when the current passing through it was small. The conductivity was found to become very large when the current was increased, so that a considerable amount of heat was developed.

But it should be added that the assumption that the ultra-violet light causes the molecules to become more easily ionized by impact would also explain any difficulties. We not only have high temperatures, but also ultra-violet light in all forms of the arc. This is especially true of the mercury arc, where ions are formed with exceptional ease. Thus, when the current increases, the amount of this light increases, which could account for the greater ease of ionization with larger currents. With increase in pressure there is an increase in the voltage, the amount of energy used in the arc increases, and no doubt the amount of ultraviolet light also becomes greater, which would account for the ionization by impact regardless of the small difference of potential through which the electrons move.

Thus, either of these explanations would seem reasonable and no fact is known to the writer which would enable one to decide between them. It is indeed altogether probable 
that both of these causes may aid in making it easier for electrons to ionize by impact.

We may then sum this up by saying that the direct cause of ionization in the arc is the impact of electrons on the molecules of the gas and that this ionization is aided either by the high temperature of the arc, or by the presence of some radiation, such as ultra-violet light, or by both of these.

An explanation has been given by Sir J. J. Thomson ${ }^{1}$ for the large discharge passing from hot calcium oxide when in a vacuum, in which he assumes that repeated bombardment of molecules by electrons will break the molecules apart, even when one impact would be quite unable to do so. Evidently the same explanation could be given for the ionization in the arc. There are, however, serious objections to this explanation in the case of discharge from hot calcium oxide ${ }^{2}$ and until further evidence is found in favor of that view it would seem to be a less probable explanation for the ionization of the arc than that which has been suggested.

Velocity of the Ions. - The current through the arc is carried very largely by electrons. These move much more rapidly than positive ions, since their masses are much smaller, while their charges are the same. We do not, however, have any data as to the exact values of the velocities of either the positive or negative ions in the arc. As far as the carbon arc is concerned we do not have any experiment showing definitely whether the positive or negative ions move the more rapidly. There have been several different methods used for the purpose of determining this question, but none of them is entirely satis-
1 Nature, 73, 495; 1906.
2 Phys. Rev., 32, 492; IgII. 
factory. The first of these was one used by myself where the positive ions were drawn from an arc by a charged cylinder placed about the arc. In this experiment the positive ions were found to have a greater velocity than the negative ones. ${ }^{1}$ However, the positive ions probably came from the hot positive electrodes and showed nothing concerning the ions in the arc itself. ${ }^{2}$

That this is the correct interpretation appears from the work of Merritt and Stewart who showed that when ions are blown from an arc by a draft of air and their velocities tested a few centimeters from the arc the negative ions have the greater velocities. ${ }^{3}$ The same thing has since been shown by myself ${ }^{4}$ and also by McClelland, ${ }^{5}$ who believed that it proved that the negative ions had the greater velocity not only outside of the arc, but also within it.

A third method of attacking this problem was given by Swinton. ${ }^{6}$ In his experiment one of the carbons was hollow and an insulated Faraday cylinder of brass was placed within this. It was found that the cylinder became charged positively as compared with the electrode in which it was placed when this was the cathode, and negatively when it was the anode, and that the negative charge was acquired more rapidly than the positive. From this Swinton concluded that the negative ions moved the more rapidly. However, this effect might easily be due to the fact that the difference of potential between the vapor and the anode is greater than that between the vapor and the cathode. $^{7}$

1 Phys. Rev., I2, I37; 190r.

2 Phys. Rev., 15, 345; 1902.

${ }^{5}$ Proc. Camb. Phil. Soc., 10, 24I; 1899.

3 Phys. Rev., 7, 129; 1898.

${ }^{6}$ Proc. Roy. Soc., 76 A, 553; 1905.

- Phys. Rev., 12, 147; 1901.

7 Phys. Rev., 24, 506; 1907. 
Still another method was given by Stark, Retschinsky, and Schaposchnikoff. ${ }^{1}$ They measured the drop in potential near secondary electrodes placed in the arc, but such measurements in the carbon arc are complicated by the high temperatures of the exploring electrodes and by the difference of potential which exists between such electrodes and the surrounding gas, so that even these measurements can not be considered as giving a definite proof of the relative velocities of the negative and positive ions in the carbon arc.

When we turn to the mercury arc we have more definite data. The measurements of Stark, Retschinsky, and Schaposchnikoff were here made with cold exploring electrodes and there would seem to be no question but that they showed that the negative ions had the greater velocity. The same thing was shown by Schenkel by means of the Hall effect (p. Ior), although the values given by him to the velocity of the negative ions depended on certain appearances which are not sufficiently definite to give exact data.

In addition to these measurements on the mercury arc there is indirect evidence furnished by the very great velocity of the negative ions in the discharge from hot platinum and from hot lime in a vacuum and by the fact that in the flame where the temperature is still much lower than in the arc the velocity of the negative ions is greater than the positive, so that in all probability in all forms of the arc the velocity of the negative ions is much greater than that of the positive. ${ }^{2}$

1 Ann. d. Phys., 18, 230; 1905.

${ }^{2}$ Certain theoretical considerations which will be published shortly in the Physical Review lead to the same conclusion. 
While suggestions have thus been made as to the cause of the ionization between the electrodes, not even a beginning has been made toward a quantitative analysis of the amount of the electric force. Not even in the mercury arc do we know the absolute velocity of the ions, the potential difference for ionization by impact, the rate of recombination, or the mean free path of the ions, and yet all of these quantities must be known in order to give an explanation of the magnitude of the electric force.

Action in Arc not the Same as in an Electrolyte. - It should be clearly understood that the explanations here offered are not in any way based on the idea that the current in the arc is carried by particles driven off from the electrodes. If the current were carried by particles the spectrum of the vapor of the arc would be a continuous spectrum instead of the line spectrum which is actually found.

Neither is it possible that the current is carried by atoms or molecules driven off from the electrodes as in electrolytes. It has been shown, for example, by Matthies ${ }^{1}$ that the current in the mercury arc is carried by electrons and not by charged atoms. Both the pressure on the anode and the behavior of the current flowing to an exploring electrode placed in the vapor indicate this.

The same thing is shown by the experiments performed by Weedon. ${ }^{2}$ He found that a copper cathode kept cool by water does not lose I/I 500 of what it should lose, if Faraday's law applied, and that the gain on the anode was very slight.

It has been thought by some that disintegration, such as is produced by boiling or by oxidation, must occur at

1 Ann. d. Phys., 37, 738; 1912.

${ }_{2}$ Paper presented at Amer. Electrochem. Soc., in 1904. 
the electrodes. There is no question but that such action at the cathode makes it easier for the current to flow, as has already been shown, but the experiments of Weedon show that this is not essential.

There has been some discussion as to whether the current is carried by the vapors which come from the anode or by those coming from the cathode. Steinmetz $z^{1}$ says that it is carried by a blast from the cathode. Blondel ${ }^{2}$ believes that it is carried by material coming from the anode. It would seem that neither of these views is entirely correct. The current is carried by that part of the vapor between the electrodes which is the most conducting. In some cases this vapor comes largely from the anode, in others from the cathode. In the carbon arc where the anode is the hotter the anode sends more conducting vapor into the arc than the cathode does. In Steinmetz' arc between a copper anode and a magnetite cathode the copper is comparatively cold and sends no vapor into the arc, while the cathode sends into it vapor that is highly conducting.

Variations of the Cathode Drop. - There has thus been outlined a theory of the arc as applied to the simplest form; namely, the arc between two electrodes which are alike, and where the gas of the arc comes from the electrodes. An example of this is the mercury arc in which both electrodes are mercury and the gas is mercury vapor. But this form is exceptional in its simplicity. With the majority of arcs there is a combination of gases to be considered and a chance for chemical action which may modify to a large extent the electrical conditions. An example of this is the open carbon arc where oxidation continually occurs.

1 Trans. Intern. Elec. Cong., St. Louis, 2, 711; 1904.

2 Trans. Intern. Elec. Cong., St. Louis, 2, 750; I904. 
An illustration of the effect produced by changing some of the conditions is shown when different metals are used for the cathode of an arc in a vacuum. As has been stated, it is difficult to maintain such an arc when the metal used for the cathode has a high melting point, and in general the higher the melting point of the cathode metal, the greater the cathode drop which is necessary. The difficulty here is not altogether due to the heat being conducted away from the hot point of the cathode, for the thermal conductivity of platinum and lead are nearly the same, yet lead can easily be made the cathode, while platinum can not be so used except with high voltages. The thermal conductivity of zinc is greater than that of iron, yet zinc may be the cathode of an arc in a vacuum, while iron can not.

Whether the difficulty is due to the hot point on the cathode being too cool to give off electrons in abundance, or to a loading of these ions with a condensing vapor we can not at present say. That the vapor does in some cases condense on the negative ions is known to be a fact and it may well be the cause of the difficulty here.

Whatever its cause, it is apparently obviated when there is a chance for chemical action at the cathode. Thus, it is easy to maintain an arc with the oxide of a metal for the cathode, and also with metals in air, and with the greater number of them in nitrogen. There is no great difficulty in maintaining one between graphite or carbon terminals in hydrogen, while it is very difficult to do so between copper or iron terminals in that gas.

Apparently it is easier to maintain an arc when there is either chemical action or ebullition at the cathode, as if ionization could occur more easily when physical or chemical change is occurring. Even with carbon in hydrogen 
the carbon usually loses weight as if some chemical or physical change were taking place.

Again, there is a change in the cathode drop when the current changes. For example with the carbon arc in air the greater the current flowing the less the cathode drop. This is probably due to the increase in the amount of heat developed near the cathode. Heat at this point will do one of two things. It will either raise the temperature of the cathode, or if the cathode is at the sublimation point, it will increase the amount of vapor sent into the arc. If the cathode becomes hotter, ionization at its surface will occur with less vigorous impact and a smaller cathode drop will be sufficient to give this impact. If on the other hand the point of sublimation has been reached, and more vapor is produced, we should again expect a smaller cathode drop for we have already seen that the cathode drop is least when the cathode is sending vapor into the arc, as it does, for example, in the mercury arc.

It is indeed more difficult to explain the fact that the cathode drop of the mercury arc does not appear to change when the current is changed (p. 96). It may be, however, that here we have so much vaporization at all times, that a limited amount more or less does not produce any appreciable effect. It would, however, be surprising if one should find that the cathode drop did not increase somewhat when very small currents were used.

The pressure of the surrounding gas also affects somewhat the value of the cathode drop, the drop becoming larger as the pressure increases. This is no doubt due to the more rapid dissipation of the heat caused by the greater number of molecules and the necessity of supplying a correspondingly greater amount of heat to the cathode. It may 
also be that the increase in the number of molecules impedes the movement of the ions and thus makes necessary a greater drop in potential.

Variations in the Anode Drop. - So long as the anode is kept cool, it may be changed without producing any appreciable effect in the anode drop. When it is heated two effects may occur. The first of these was pointed out by Stark, Retschinsky and Schaposchnikoff ${ }^{1}$ who called attention to the fact that when a metal becomes very hot it tends to send out negative ions, and if the metal is the anode, these ions must be driven back again into the metal. It would, therefore, be necessary to have a larger anode drop when the metal is hot than when it is cool (p. 97).

In the second place the conditions become quite different when the anode becomes sufficiently hot to vaporize. This is especially true, if the vapor which the anode sends into the arc is different from the gas existing there when the anode is cool. The anode drop may then change by a very appreciable amount. Such a change no doubt occurs in the iron arc when it passes from the first to the second stage (p. 65).

The anode drop is also very greatly affected by any other change in the kind of vapor in the arc. As we have seen (p. 6o), the introduction of a salt, such as sodium carbonate, causes the anode drop to be very much lower. Again, if the cathode is changed so that a different vapor comes from it into the arc, the anode drop is changed. Thus I found that with cored carbon for both anode and cathode the anode drop was 38 volts. With the same current and length of arc and the same anode, but with iron for the cathode the anode drop was I4 volts. Apparently the 1 Ann. d. Phys., 18, 219; 1905. 
negative ions in the iron vapor do not require so large a potential difference in order to ionize the atoms at the surface of the anode.

The anode drop in the carbon arc decreases when the current increases. As was the case with the cathode drop this may be due to an increase in the temperature of the anode or to more rapid vaporization of the carbon. Those who have made determinations of the temperature of the anode differ as to whether this temperature depends on the amount of current flowing or not (p. 45), so that we can not tell which of these two phenomena should be expected to follow when there is an increase in the current. Either of them would be followed by a lowering of the anode drop.

The cause of the increase of the anode drop when the pressure of the gas is increased is no doubt the same as the corresponding increase in the cathode drop. The greater the pressure the faster the heat is taken away by molecules of the gas and the more heat must be produced by the current. Again, the greater the number of molecules in the neighborhood of the anode the more the ions passing to and from it will be retarded.

Variations in the Electric Force through the Arc. The electric force through the arc is changed very much when the kind of gas is changed, as when a salt is introduced into the arc or when the anode becomes sufficiently hot to send vapor into the arc. This force also depends on the kind of gas in which the arc is placed. Thus Stark, Retschinsky and Schaposchnikoff found that the electric force between either copper or carbon electrodes was greater when the carbons were in hydrogen than when they were in carbon dioxide.

The cause of the change in some cases is the greater ease 
with which some substances are ionized than other substances. Thus sodium carbonate, when at a high temperature, appears to be much more easily ionized than air. In other cases the change may be due to the greater rapidity with which heat is conducted away by the gas as with an arc placed in hydrogen. It is probable that a large part of the decrease in the potential difference between the terminals of an iron arc when it passes from the first to the second stage is due to the greater ease with which the gas from the vaporizing metal anode can be ionized.

Effect Produced by Heating the Cathode. - To produce the high temperature of the cathode there must be an expenditure of electrical energy in its neighborhood. The rate at which this is done equals the cathode drop times the current. It is natural to expect that if the cathode is cooled artificially, it will require a greater expenditure of energy to maintain the needed temperature, and to do this with a given current the cathode drop must be greater. Similarly, with any method of heating we should expect the cathode drop to be less.

But it was found (p. 48) that cooling the cathode produced but a slight effect on the cathode drop, while it had a very appreciable effect on the anode drop and on the fall of potential through the arc. A possible explanation for this is that it is a mistake to suppose that the temperature of the cathode must be kept at a certain definite value. It is probable that there may be conditions where this temperature is lower than that which is commonly found. However, when the temperature of the cathode is lowered, it sends out fewer electrons and as a result the remaining parts of the arc have a smaller conductivity, so that we get the effects which have been observed. 



\section{INDEX}

(The numbers refer to pages.)

A

Abey and Festing, 43 .

Alternating-current arc:

with carbon electrodes, ro5.

characteristic curves for, 106.

current and potential difference for different phases of, ro7. oscillographs for, rog.

dynamical characteristic curves for, Iro.

anode and cathode drops in, II2.

phase difference in, II3.

between metals, II4.

in a vacuum, $1 \mathbf{r} 7$.

between unlike terminals, I 18 .

in other gases than air, 1 I9.

candle power of, $\mathbf{I} 35$.

photometry of, $¥ 35$.

Amalgams, arc between, Io2.

Andrews, 48.

Anode:

definition of, 4 .

temperature of, 40,165 .

ionization at, 162, I68.

effect produced by heating, $\mathbf{1 8 5}$.

See also Electrodes and Crater.

Anode drop:

definition of, 22.

in carbon arc in air, 24 .

in flaming arc, 60 .

with copper electrodes, 65 .

with graphite electrodes, 66 .

with metal electrodes, 66 .

with unlike electrodes, 72 .
Anode drop:

in a vacuum, 78 .

in mercury arc, 97.

in alternating-current arc, $\mathbf{I} 2$.

theory of, $x 68$.

variations in, 183 .

Appearance of arc:

carbon arc in air, 3 .

flaming arc, 54 .

magnetite arc, 67 .

arc in a vacuum, 75 .

mercury arc, 88.

alternating-current arc, 105.

Arnold, Cady and, 64, I45.

Arons, 30, 37, 71, 82, 87, 92, 96, ro3, I 14, I 6 , I64.

Austin, I53.

Ayrion, 3I, I3r.

Ayrion, Mrs., 10, 21, 24, 48, I30.

B

Back E.M.F., see Counter E.M.F.

Baeyer, Gehrke and, ro3.

Baldwin, Miss, 143 .

Banderet, 66.

Becknell, 38.

Becquerel, 4r.

Bedell, I24.

Blondel, I6, 37, 50, II9, 130, т32, I39, I70, I80.

Blondel lamp, candle power of, $\mathbf{1} 39$. Boston flaming arc, candle power of, I 40.

Braun, 147. 
Bremen, 56.

Bremen lamp, candle power of, 139 . Brilliancy of crater, 132 .

Bron, Guye and, II5.

Burgess, Waidner and, 43, 173 .

C

Cady and Arnold, 64, I45.

Cady and Vinal, 65.

Calcium fluoride in flaming arc, 56 .

Calcium oxide at cathode, 82 .

Candle power:

of direct-current carbon arc, 128.

and area of crater visible, I29.

as affected by length of arc, current, etc., I30.

of flaming arcs, 133 .

of mercury arc, 134 .

of alternating-current arcs, I35.

comparison of, $\mathrm{r} 39$.

Carbon arc in air:

characteristic curves for, 9 .

E.M.F. required, $\mathbf{I 6 .}$

potential in different parts of, 22.

cathode drop in, 22.

anode drop in, 24.

electric force in, 24 .

counter E.M.F. of, 25.

temperature of, 40.

with alternating currents, 105.

candle power of, $\mathrm{I} 28$.

Carbon arc in vacuum:

appearance of, 75 .

voltage with different pressures, 78 . anode and cathode drops in, 78 . voltage with different lengths, 79 . temperature of, $8 \mathrm{r}$. with alternating currents, II 7 .

Carbon electrodes: manufacture of, 8 .
Carbon electrodes:

solid, 8.

cored, 9 .

diameter of, and candle power, I37.

Carbon dioxide, arc in, 85 .

Carbon monoxide, arc in, 85 .

Carbone lamp, I33.

Casselmann, 55 .

Cassuto, Stark and, I63.

Cathode:

definition of, 4 .

area of, 49.

ionization at, 161,165 .

must be hot, 162 .

temperature of, 165 .

effect produced by heating, 185 .

Cathode drop:

definition of, 22.

in carbon arc in air, 22.

in flaming arc, 60 .

with copper electrodes, 65 .

with graphite electrodes, 66 .

with unlike electrodes, 72 .

in a vacuum, 78 .

in mercury arc, 96 .

in alternating arc, $\mathbf{1} 2$.

theory of, $\mathbf{x} 66$.

variations in, I8o.

Characteristic curves:

definition of, I2.

for arcs between solid carbons, I3.

for arcs between cored carbons, I4. statical, I6.

dynamical, I6, Iro, I5I.

for enclosed arc, 50.

for flaming arc, 58 .

for arc between metals, 62 .

for magnetite arc, 67 .

for iron-titanium arc, 69 .

for mercury arc, 93 . 
Characteristic curves:

for alternating-current arc, I06, IIO.

Cheneveau, 5 r.

Color of:

flaming arc, 54 .

mercury arc, 88.

carbon arc, 126, 142.

Conductivity of arc, see Resistance.

Consumption of electrodes, 49, I39.

Cooling of electrodes, 46,185 .

Cooper Hewitt, 87, 95, I03, I 20.

Cooper Hewitt mercury vapor lamp, 89.

Copper electrodes, arc between, 62 .

Corbino and Liga, 37.

Cored carbons:

manufacture of, 9 .

characteristic curves for, I4.

anode drop with, 24 .

cathode drop with, 24 .

Cores, composition of, 9 .

Counter E.M.F. of arc, Io, 25.

Crater:

definition of, 4 .

temperature of, 40.

area of, and current, 48.

area of, and candle power, $\mathbf{2} 29$.

intrinsic brightness of, 132 .

Cunningham, 165.

Current:

and potential difference, see Characteristic curves.

oscillation of, with hissing arc, 21 . with different phases of alternating-current arc, I07.

rectifier, 120.

and candle power, $\mathbf{3}$.

\section{D}

Davis, 159, 173.

Davy, 6, 50, 62, 74 .
Definition of:

arc, I.

anode, 4.

cathode, 4.

characteristic curves, I 2.

ions, 156 .

electrons, ${ }_{5} 5$.

De la Rive, 46, 74.

De la Rue and Miller, 74.

Dewar, 4I, $5^{\mathrm{I}}$.

Discharge in vacuum compared with arc, 2.

Discovery of:

carbon arc, 5 .

mercury arc, 88.

Distribution of light with:

direct-current carbon arc, I 28.

alternating-current carbon arc, I36.

Dubs, 38 .

Duddell, I5, 28, 32, II6, I36, I48, I52.

Duddell and Marchant, 22, II 2.

Duncan, Rowland and Todd, 73.

Dynamical characteristic curves, 16 , IIO, 15 I.

Dyott, 68 .

\section{$\mathrm{E}$}

Edlund, 9, 25, 35, 62.

Efficiency of arc, see Candle power.

Electric force in arc:

in air, 24.

in vacuum, 78 .

mercury arc, 97.

theory of, I73.

variations of, $\mathrm{I} 84$.

Electrodes:

arc between carbon, 8 .

manufacture of, 8,56 .

consumption of, $49, \mathbf{r} 39$. 
Electrodes:

light from, 129.

discharge of ions to, I70.

See also Metal electrodes, Oxides,

Magnetite, Electrolyte, and Un-

like.

Electrolytes:

arc between, 69 .

arc not like, 179 .

Electrons:

definition of, I56.

coming from within metal, $\mathrm{I}_{3}$.

E.M.F.:

counter, IO, 25.

required for arc, $\mathbf{1} 6$

"forward," 33.

residùal, 35 .

Enclosed arc:

description of, 49 .

characteristic curves for, 50 .

candle power of, I37, I39.

Exploring electrodes, 22.

\section{F}

Feige, Urbain, Scal and, ro3.

Fery, 43, 93.

Festing, Abey and, 43 .

Firth, 3I.

Firth and Rogers, 22, 32.

Fitzgerald, Wilson and, 45, 73.

Flaming arc:

appearance of, 54 .

history of, 55 .

composition of electrodes with, 56 .

advantages of, 57 .

characteristic curves for, 58 .

anode and cathode drops in, 60 .

with alternating currents, Iog.

photometry of, I32.

candle power of, 133 .

Fleming, 23.
Fleming and Petavel, I27, 137.

Foley, 143.

"Forward," E.M.F., 33.

Freedman, 63.

\section{G}

Gas between electrodes: temperature of, 40. ionization of, $\mathbf{r} 7 \mathrm{r}$.

Gases, arc in various, 83, IIg.

Gehrke and Baeyer, I03.

General Electric Company: mercury vapor lamp, 90 . mercury arc rectifier, I22.

Georges, 136 .

Glow discharge, 2.

Gould, I18.

Granqvist, 37, 49, II4.

Grau and Russ, 63, 85 .

Gray, Wilson and, 4I.

Grove, 69 .

Guye and Bron, Ir5.

Guye and Monash, II5.

Guye and Zebrikoff, 63.

$\mathrm{H}$

Hagenbach and Veillon, 64 .

Hall effect in mercury arc, $\mathrm{IO}_{2}, \mathrm{r} 78$.

Heinke, 34 .

Herizfeld, I37.

Heubach, ro6, II3.

Hewitt, see Cooper Hewitt.

Hissing arc:

voltage of, 2 I.

cause for, 21 .

with metals, 65 .

History of arc, 5 .

Hittorf, 165 .

Hoerburger, 79.

Horton, I60.

Hotchkiss, 38. 
Hull, I44.

Humphreys, I45.

Hydrogen, arc in, 83 .

\section{I}

Intrinsic brightness of crater, 132 .

Ionization:

by impact, 158 .

by hot solids, 159 .

at surface of cathode, I6r, I62, 165 .

at surface of anode, I62, I68.

of gas between electrodes, I7I.

by ultra-violet light, 172 .

Ions:

velocity of, Ior, 176 .

definition of, 156 .

causes producing, 158 .

discharge of, to electrodes, 170.

Iron electrodes, 64.

Iron-titanium arc:

characteristic curves for, 69 .

candle power of, I39.

\section{J}

Jamin and Manewvier, II4, II8.

Janet, 152.

Joubert, 107.

K

Kayser, 144.

Knipp, 92, 96, 97.

Kuch and Retschinski, 100, 135 .

Kuch, Stark and, $8 \mathbf{I}$.

L

Lamp, mercury vapor, 89 .

Lange, Gerirude, II 2.

Le Chatelier, $4 \mathrm{I}$.

Lecher, 24, 37, 46, 63, 66.

Lenard, $\mathbf{1 4 4 .}$
Length of arc:

and candle power, $\mathrm{I}_{3}$.

and potential difference, see Characteristic curves.

Le Roux, 37, 106.

Liga, Corbino and, 37.

Light from arc:

quality of, see Color.

quantity of, see Candle power.

Luggin, 24, 28, 31, 37, 60, 163.

Luminous vapor from mercury arc, 99.

Lummer and Pringsheim, 43.

\section{M}

McClelland, $\mathrm{I} 77$.

Magnetic field:

carbon arc in, 6, 50 .

whistling arc in, $15 \mathrm{I}$.

Magnetite arc: characteristic curves for, 67 . candle power of, I39, I40. ionization in, 180 .

Mahlke, 9, 56.

Malcolm and Simon, 15, 48, 64, 83. Manewier, Jamin and, II4, II8.

Manufacture of carbons, 8 .

Marchant, r33.

Marchant, Duddell and, 22, II2.

Marks, 49.

Malthies, 179.

Matthews, 137 .

Mean free path of ions in arc, 173 .

Mean spherical candle power, I28.

Mercury arc:

discovery of, 88 .

commercial form of, 89 .

temperature of, 9 r.

characteristic curves for, 93 .

pressure in, 94.

cathode drop in, 96 . 
Mercury arc:

anode drop in, 97.

electric force in, $9 \%$.

in quartz tubes, 100 .

velocity of ions in, ror.

modification of, to produce white

light, 102.

rectifier, $\mathbf{I} 20$.

with alternating currents, 124 .

photometry of, 134 .

candle power of, $\mathrm{I}_{34}, \mathrm{I}_{39}, \mathrm{I}_{4}$.

Merritt and Stewart, I77.

Metal electrodes:

arc with, 6r.

characteristic curves with, 62 .

hissing arcs with, 65 .

anode and cathode drops with, 65 . different metals used for, 66, 8I, II 5 .

in a vacuum, $8 \mathrm{x}$.

with alternating currents, $\mathrm{II}_{4}$. photometry of, 134 .

Melleucci, 62.

Microphone and wireless telephony,

154 .

Milkiewicz, 38 .

Moissan, 40.

Monash, Guye and, II5.

Morris, 51, x20.

Müller, de la Rue and, 74.

\section{$\mathrm{N}$}

Negative electrode, see Cathode.

Negative ions, 157.

Negative resistance, $3 \mathrm{r}$.

Nitrogen arc in, 84.

Non-arcing metals, II4.

Norden, I24.

Olivette, 38.

Oscillograph, 38 .
Oscillographs of:

hissing arc, 21 .

arc in magnetic field, 5 r

alternating-current arc, Iog.

Oxides, arc between, 67 .

Oxygen, arc in, 85 .

$P$

Petavel, 4r; $\mathrm{I}_{32}$.

Petavel, Fleming and, I27, 137 .

Phase difference between current and voltage with alternatingcurrent arcs, II3.

Photometry:

difficulties in, 126 .

of direct-current carbon arc, I28.

of flaming arc, $\mathrm{r}_{32}$.

of metal arcs, I34.

of mercury arc, 134 .

of alternating-current arc, I35.

See also Candle power.

Planck's law, 43.

Pollak, 96, 98, I75.

Pollock, 34 .

Pollock and Ranchaud, 52.

Positive electrode, see Anode.

Positive ions, 157 .

Potassium alloy, 82 .

Potential difference between terminals of:

carbon arc, 9.

arc in vacuum, 78,79 .

arc in hydrogen, 86.

See also Characteristic curves.

Potential difference with different phases of alternating-current arc, 107.

Potential gradient, see Electric force.

Potential within arc, 22, I60.

Poulsen, I5I.

Power used in arc, 14 . 
Pressure in mercury arc, 94.

Pressures greater than one atmosphere, 73 .

Pringsheim, Lummer and, 43.

Puccianti, 144 .

Pyrometer, 44.

\section{Q}

Quartz tubes for mercury arc, 100.

\section{$\mathbf{R}$}

Ranchaud, Pollock and, 52.

Rasch, 67.

Rectifier, mercury arc:

three-phase, $\mathbf{r} 2$.

single-phase, 122 .

Reich, 45, 49, I65.

Residual E.M.F., 35 .

Resistance:

of arc, 9, 28 .

in series with arc, 22.

negative, 3 I.

after removal of E.M.F., 38.

Retschinski, Kuch and, 100, I35.

Retschinski, Stark and Schaposchnikoff, $83,96,97,168,178,183$.

Rey, 132.

Richardson, O. W., I59, I64.

Rogers, Firth and, 22, 32.

Rosetti, 4I.

Rossler and Wedding, I37.

Rowland, Duncan and Todd, 73.

Ruhmer, ${ }_{4} 8$.

Russ, Grau and, 63, 85 .

Ryan, r40.

\section{S}

Sahulka, 69, II8.

Salts, effect of, in arc, 55 .

Scal, Urbain, and Feige, ro3.
Schaposchnikoff, Stark, Retschinski and, $83,96,97,169,178,183$.

Schenkel, Іог, 178.

Schulize, 48, 67 .

Sharpe, 133 .

Silent arc, $2 \mathrm{I}$.

Simon, 16, 109, 146, I51.

Simon, Malcolm and, ${ }_{5}, 48,64,83$.

Skinner, 168.

Sodium alloy for electrodes, 82 .

Solid carbons, 9 . characteristic curves with, I3.

Spark compared with arc, 2.

Spectrum of carbon arc, $\mathbf{I}_{42}$.

Stability of arc, conditions for, 18.

Stark, 82, 99, 156, 164 .

Stark and Cassuto, 163.

Stark and Kuch, 81.

Stark, Retschinski and Schaposchnikoff, $83,96,97,168,178,183$.

Static converter, I 20.

Statical characteristic curves, 16.

Stefan-Boltzmann law, 42.

Steinmetz, II4, I45, I80.

Stenger, 37, 74 .

Stewart, Merritt and, 177.

Sulphur dioxide, arc in, 85.

Swendler, 29.

Swinton, 177 .

\section{$\mathrm{T}$}

Telephony, wireless, and arc, I46.

Temperature of:

gas in carbon arc, 40.

anode of carbon arc, 40.

variation of, 44 .

arc in vacuum, $8 \mathbf{r}$.

mercury arc, $9 I$.

Terminals, see Electrodes.

Theory of arc, ${ }_{5} 6$. 
Thomson, E., $x 48$.

Thomson, Sir J. J., I56, I70, I76.

Titanium arc, 68.

Tobey and Walbridge, 107.

Todd, Duncan, Rowland and, 73.

Tommasi, 48 .

Townsend, 158, 173 .

Trotter, 5I, 129.

\section{U}

Ultra-violet light:

produced by arc, roo.

ionization by, $x 72$.

Unlike electrodes, 69, II8.

Uppenborn, 24.

Upson, 63.

Urbain, Scal and Feige, ro3.

\section{V}

Vacuum, arc in, 74, 8r, II 7 .

Van Breda, 74.

Variation in temperature of arc, 44.

Veillon, Hagenbach and, 64 .

Velocity of ions in: mercury arc, IOI. carbon arc, I77.

Very, 43.

Vinal, Cady and, 65.

Violle, 42.
Voltage of arcs, see Potential difference between terminals.

Von Lang, 29, 63.

\section{W}

Waidner and Burgess, 43, I73.

Walbridge, Tobey and, 107.

Walker, 50.

Wanner, 43.

Wave form and candle power, $\mathbf{1} 37$.

Wedding, 133 .

Wedding, Rossler and, 137.

Weedon, 69, 85, 179 .

Wehnelt, 159.

Weintraub, 82, I22.

Wey, 88.

Whispering arc, 146 .

Whistling arc, I48.

Wien's law, 42.

Wild, 36 .

Wilde, $5 \mathrm{r}$.

Wills, 92.

Wilson and Gray, 4r.

Wilson and Filzgerald, 45, 73.

Wilson, H. A., I60.

Wilson, W. E., 45, I32.

Wireless telephony, use of arc in, 146 .

Wood's metal, arc with, 82.

Wurts, II4.

$\mathrm{Z}$

Zebrikoff, Guye and, 63. 


\section{LIST OF WORKS \\ ON}

\section{ELECTRICAL SCIENCE}

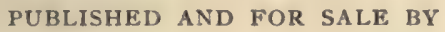

\section{VAN NOSTRAND COMPANY, 23 Park Place, New York.}

ABBOTT, A. V. The Electrical Transmission of Energy. A Manual for the Design of Electrical Circuits. Fifth Edition, enlarged and rewritten. . With many Diagrams, Engravings and Folding Plates. 8vo., cloth, 675 pp. . . . . . . . . . . . . . . . . . . Net, $\$ 5.00$

ALEXANDER, J. H. Elementary Electrical Engineering in Theory and Practice. A class-book for junior and senior students and working electricians. Illustrated. 12mo., cloth, $208 \mathrm{pp} \ldots \ldots \ldots \ldots \ldots 2.00$

ANDERSON, GEO. L. Handbook for the Use of Electricians in the operation and care of Electrical Machinery and Apparatus of the United States Seacoast Defenses. Prepared under the direction of Lieut.-General Commanding the Army. Illustrated 8vo,, eloth, 161 pp.............................. \$3.00

ARNOLD, E. Armature Windings of Direct-Current Dynamos. Extension and Application of a general Winding Rule. Translated from the original German by Francis B. DeGress. Illustrated. 8vo. cloth, 124 pp............................ \$2.00

ASHE, S. W. Electricity Experimentally and Practically Applied. Second Edition. 422 illustrations. $12 \mathrm{mo}$., cloth, $375 \mathrm{pp}$..Net, $\$ 2.00$ 
ASHE, S. W., and KEILEY, J. D. Electric Railways Theoretically and Practically Treated. Illustrated. 12mo., cloth.

Vol. I. Rolling Stock. Second Edition. $285 \mathrm{pp} . \ldots \ldots \ldots$.... Net, $\$ 2.50$

Vol. II. Substations and Distributing Systems. $296 \mathrm{pp} \ldots$... Net, $\$ 2.50$

ATKINSON, A. A. Electrical and Magnetic Calculations. For the use of Electrical Engineers and others interested in the Theory and Application of Electricity and Magnetisn. Fvurth Edition, revised Illustrated. $12 \mathrm{mo}$, cloth, $310 \mathrm{pp} . \ldots \ldots \ldots \ldots \ldots \ldots$. . . . . $\$ 1.50$

ATKINSON, PHILIP. The Flements of Dynamic Electricity and Magnetism. Fourth Edition. Illustrated. 12mo., cloth, 405 pp. .\$2.00

-Elements of Flectric Lighting, including Electric Generation, Measurement, Storage, and Distribution. Tenth Edition, fully revised and new matter added. Illustrated. $12 \mathrm{mo}$, cloth, $280 \mathrm{pp} . \ldots \ldots \ldots . \$ 1.50$

- Power Transmitted by Electricity and Applied by the Electric Motor, including Electric Railway Construction. Illustrated. Fourth Edition, fully revised and new matter added. $12 \mathrm{mo}$., cloth, $241 \mathrm{pp} . \quad \ldots \$ 2.00$

AYRTON, HERTHA.. The Electric Arc. Illustrated. 8vo., cloth, 479 pp................................. $\$ 5.00$

AYRTON, W. E. Practical Electricity. A Laboratory and Lecture Course. 300 illustrations. $6 \times 8 \%$, cloth, $562 \mathrm{pp} . . . .$. Net, $\$ 3.00$

BAKER, J. T. The Telegraphic Transmission of Photographs. 63 illustrations. $12 \mathrm{mo}$. , cloth, $155 \mathrm{pp} \ldots \ldots \ldots \ldots \ldots \ldots$............... $\$ 1.25$

BEDELL, FREDERICK. Direct and Alternating Current Manual. With directions for testing and a discussion of the theory of electrical apparatus. Second Edition, greatly enlarged. Assisted by C. A. Pierce. Illustrated. 6x9, cloth, $373 \mathrm{pp} \ldots \ldots \ldots \ldots \ldots$. Net, $\$ 2.00$

BEDELL, F. \& CREHORE, ALBERT C. Alternating Currents. An analytical and graphical treatment for students and engineers. Fifth Edition. 112 illustrations. 8vo., cloth, 325 pp... Net, $\$ 2.50$

BLAINE, ROBERT G. The calculus and Its Applications. A practical treatise for beginners especially engineering students. 79 illustrations. $12 \mathrm{mo}$., cloth, 330 pp...................... $\$ 1.50$

BONNEY, G. E. The Electro-Plater's Hand Book. A Manual for Amateurs and Young Students of Electro-Metallurgy. Fourth Edition, enlarged. 61 Illustrations. $12 \mathrm{mo}$., cloth, $208 \mathrm{pp} . . \ldots \ldots \ldots . \$ 1.20$ 
BOTTONE, S. R. Magnetos For Automobilists; How Made and How Used. A handbook of practical instruction on the manufacture and adaptation of the magneto to the needs of the motorist. Second Edition, enlarged. 52 illustrations. $12 \mathrm{mo}$., cloth, $118 \mathrm{pp} \ldots \ldots$... Net, $\$ 1.00$

BOWKER, WM. R. Dynamo, Motor, and Switchboard Circuits for Electrical Engineers: a practical book dealing with the subject of Direct, Alternating, and Polyphase Currents. Second Edition, greatly enlarged, 130 illustrations. 8vo., cloth, $180 \mathrm{pp} \ldots \ldots \ldots$.... Net, $\$ 2.50$ BREW, W. Three-Phase Transmission. A practical treatise on the economic conditions governing the transmission of electric energy. 83 illustrations. $6 \times 9$, eloth, $186 \mathrm{pp} \ldots \ldots \ldots \ldots \ldots \ldots$ Net, $\$ 2.00$

BROADFOOT, S. K. Motors, Secondary Batteries and Accessory Apparatus. 16 illustrations. $16 \mathrm{mo}$,, cloth. $100 \mathrm{pp}$. '(Installation Manuals Series)..................................... 0.75

BROUGHTON, H. H. Electric Canes. Their design, eonstruction and application. 600 illustrations and plates. 120 tables, $6 \times 9$, cloth,

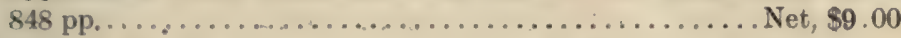

CARTER, E. T. Motive Power and Gearing for Electrical Machinery; a treatise on the theory and practice of the mechanical equipment of power stations for electric supply and for electric traction. Second Edition, revised. Illustrated. \$vo., cloth, $700 \mathrm{pp} \ldots$.... Net, $\$ 5.00$

CHILD, C. D. Electric Arcs. Experiments upon ares between different electrodes in various environments and their explanation. 58 illustrations. $5 \times 7$, cloth, 203 pp..................... $\$ 2.00$

CHILD, CHAS. T. The How and Why of Electricity: a book of information for non-technical readers, treating of the properties of Electricity, and how it is generated, handled, controlled, measured, and set to work. Also explaining the operation of Electrical Apparatus. Illustrated. 8vo., cloth, $140 \mathrm{pp} \ldots \ldots \ldots \ldots \ldots \ldots \ldots \ldots \ldots \ldots$

COOPER, W. R. Primary Batteries: their Theory, Construction, and Use 131 Itlustrations. 8vo., eloth. 32.4 pp.............. Net, $\$ 4.00$ CRAMP, W. Continuous Current Machine Design. 137 illustration. 8 vo., cloth, 240 pp............................. $\$ 2.50$

CROCKER, F. B. Electric Lighting. A Practical Exposition of the Art for the use of Flectricians, Students, and others interested in the Installation or Operation of Electric-Lighting Plants.

Vol. I. - The Generating Plant. Seventh Edition, entirely revised. Illus trated. 8vo., eloth, 482 pp...................\$3.00

Vol. II.-Distributing System and Lamps. Sixth Edition. Illustrated $8 v o .$, eloth, $505 \mathrm{pp}$. 
CROCKER, F. B., and ARENDT, M. Electric Motors: Their Action, Control, and Application. 160 illustrations. 8vo., cloth, $296 \mathrm{pp}$.

Net, $\$ 2.50$

CROCKER, F. B., and WHEELER, S. S. The Management of Elestrical Machinery. Being a thoroughly revised and rewritten edition of the authors' "Practical Management of Dynamos and Motors." Eighth Edition. Illustrated. 16mo., cloth, $232 \mathrm{pp} . . .$. Net, $\$ 1.00$

CUSHING, H. C., Jr. Standard Wiring for Flectric Light and Power Illustrated. $16 \mathrm{mo}$, leather, $156 \mathrm{pp} \ldots \ldots \ldots \ldots \ldots \ldots \ldots \ldots 1.00$

DAVIES, F. H. Electric Power and Traction. Illustrated. 8vo, eloth 293 pp. (Van Nostrand's Westminster Series.) ......... Net, \$2.00

---Foundations and Machinery Fixing. 52 illustrations. $16 \mathrm{mo}$., cloth, 146 pp. (Installations Manuals Series)............. Net, $\$ 0.75$

DEL MAR, W. A. Electric Power Conductors. 69 illustrations. 8vo., cloth, 330 pp................................... $\$ 2.00$

DEVEY, R. G. Mill and Factory Wiring. 126 illustrations. $16 \mathrm{mo}$., cloth, 209 pp. (Installation Manuals Series)........ Net, $\$ 1.00$

DINGER, Lieut. H. C. Handbook for the Care and Operation of Naval Machinery. Second Edition. 124 Illustrations. $16 \mathrm{mo}$., cloth, 302 pp..................................... $\$ 2.00$

DWIGHT, H. B. Transmission Line Formulas for Electrieal Engineers and engineering Students. 27 illustrations. 2 folding plates, $12 \mathrm{mo}$,

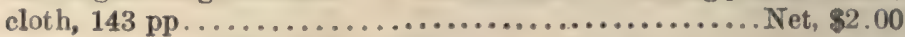

DYNAMIC ELECTRICITY: Its Modern Use and Measurement, chiefly in its application to Electric Lighting and Telegraphy, including: 1. Some Points in Electric Jighting, by Dr. John Hopkinson. 2, On the Treatment of Electricity for Commercial Purposes, by J. N. Shoolbrer. 3. Electric-Light Arithmetic, by R. E. Day, M.E. Fourth Edition. Illustrated. 16mo., boards, 166 pp. (No. 71 Van Nostrand's Science Series.)...................50 cents

EDGCUMBE, K. Industrial Electrical Measuring Instruments. Illustrated. 8vo., cloth, 227 pp..................... Net, $\$ 2.50$

ERSKINE-MURRAY, J. A Handbook of Wireless Telegraphy: Its Theory and Practice. For the use of electrical engineers, students, and operators. Fourth Edition, revised and enlarged. 200 Illustrations. $61 / 4 \times 9$, eloth, 442 pp......................... $\$ 3.50$

-Wireless Telephones and How they Work. Illustrated. $16 \mathrm{mo}$, cloth, 75 pp...............................\$1.00 
EWING, J. A. Magnetic Induction in Iron and other Metals. Third Edition, revised. Illustrated. 8 vo., eloth, $393 \mathrm{pp} \ldots \ldots$ Net, $\$ 4.00$

FISHER, H. K. C., and DARBY, W. C. Students' Guide to Submarine Cable Testing. Third Edition, new, enlarged. Illustrated. 8vo., cloth, 326 pp................................... $\$ 3.50$

FLEMING, J. A., Prof. The Alternate-Current Transformer in Theory and Practice.

Vol. I.: The Induction of Electric Currents. Fifth Issue. Illustrated. 8 vo., eloth, 641 pp.............................. $\$ 5.00$

Vol. II.: The Utilization of Induced Currents. Third Issue. Illustrated. 8vo., eloth, $587 \mathrm{pp} . . . \ldots \ldots \ldots \ldots \ldots \ldots$............. $\$ 5.00$

- Propagation of Electric Currents in Telephone and Telegraph Conductors. Illustrated. $6 \frac{1 / 2 \times 2}{11 / 2}$, eloth, $323 \mathrm{pp} . \ldots \ldots \ldots$. Net, $\$ 3.00$

- - Handbook for the Electrical Laboratory and Testing Room. Two Volumes. Illustrated. 8vo. cloth, 1160 pp. Each vol ..... Net, $\$ 5.00$

FOSTER, H. A. With the Collaboration of Eminent Specialists. Electrical Engineers' Pocket Book. A handbook of useful data for Electricians and Electrical Engineers. With innumerable Tables, Diagrams, and Figures. The most complete book of its kind ever publishe:1, treating of the latest and best Practice in Electrical Fngineering. Seventh Edition, completely revised and enlarged. Fully Illustrated. Pucket Size. Leather. Thumb Indexed. 1636 pp .......\$5.00

- Engineering Valuation of Public Utilities and Fastories. 50 blank forms. $6 \times 9$, cloth, $361 \mathrm{pp} \ldots \ldots \ldots \ldots \ldots \ldots \ldots$................. $\$ 3.00$

FOWLE, F. F. The Protection of Railroads from Overhead Transmission Line Crossings. 35 illustrations. 12mo.. cloth, $76 \mathrm{pp}$. Net, $\$ 150$.

FRITH, J. Alternating Current Design. 27 illustrations. $6 \times 9$, cloth,

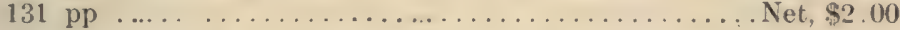

FREUDEMACHER, P. W. Electrical Mining Installations. 36 illustrations. $16 \mathrm{mo}$., cloth, $192 \mathrm{pp}$. (Installation Manuals Series) . Net, $\$ 1.00$

GANT, L. W. Elements of Electric Traction for Motormen and Others. Illustrated with Diagrams. $8 v 0$. , eloth, $217 \mathrm{pp} \ldots \ldots \ldots$ Net, $\$ 2.50$

GEAR, H. B. and WILLIAMS, P. F. Electric Central Station Distribution Systems. Their Design and Construction. 139 illustrations. 8\%o.,

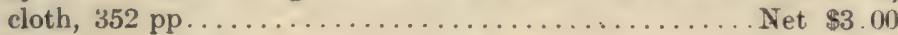

GERHARDI, C. H. W. Electricity Meters; their Construction and Management. A practical manual for engineers and students. [llustrated. 8vo., cloth, 337 pp. ..................... Net. \$4.00. 
GORE, GEORGE. The Art of Electrolytic Separation of Metals (Theoretical and Practical). Illustrated. 8vo., cloth, $295 \mathrm{pp}$.... Net $\$ 3.50$

GROTH, L. A. Welding and Cutting Metals by Aid of Gases or Electricity. 124 illustrations. 8 vo., eloth, $280 \mathrm{pp} \ldots . .$. Net, $\$ 3.00$

HALLER, G. F. and CUNNINGHAM, E. T. The Tesla High Frequency Coil; its construction and uses. $12 \mathrm{mo}$., cloth, 56 illustrations, 130 pp......................................... $\$ 1.25$

HASKINS, C. H. The Galvanometer and its Uses. A Manual for Electricians and Students. Fifth Edition, revised. Illustrated. 16mo.,

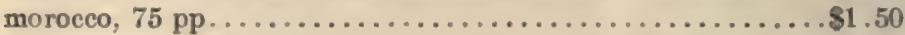

HAY, ALFRED. Principles of Alternate-Current Working. Second Edition. Illustrated. 12mo., cloth, $390 \mathrm{pp} \ldots \ldots \ldots \ldots \ldots \$ 2.00$

- Alternating Currents; their theory, generation, and transformation. Second Edition. 191 Illustrations. 8vo., cloth, 319 pp...Net, \$2.50

- An Introductory Course of Continuous-Current Engineering. Illustrated. 8vo., cloth, 327 pp...................... $\$ 2.50$

HEATHER, H. J. Electrical Engineering for Mechanical and Mining Engineers. 183 illustrations. $5 \frac{8}{4} \times 88$, cloth, $344 \mathrm{pp} . . .$. Net, \$3.50)

HEAVISIDE, O. Electromagnetic Theory. Two Volumes with Many Diagrams. 8vo., eloth, 1006 pp. Each Vol.......... Net, \$5.00

Vol. III. -529 pp......................... Net, $\$ 7,50$

HEDGES, K. Modern Lightning Conductors. An illustrated Supplement to the Report of the Research Committee of 1905 , with notes as to methods of protection and specifications. Illustrated. 8vo., cloth, 119 pp.................................. $\$ 3.00$

HOBART, H. M. Heavy Electrical Engineering. Illustrated. \&vo., eloth, 338 pp................................ $\$ 4.50$

- Design of Static Transformers. 101 illustrations. 6x9, cloth, 189 pp..................................... $\$ 2.00$

- Electricity. A text-book designed in particular for engineering students. 115 illustrations. 43 tables. 8vo., cloth, 266 pp., Net, $\$ 2.00$

-Electric Trains. 88 illustrations. 8vo., eloth, 220 pp... Net, \$2.50 --Electric Propulsion of Ships. 44 illustrations. 8qo., cloth, 167 pp. 
HOBBS, W. R. P. The Arithmetic of Electrical Measurements. With numerous examples, fully worked. Twelfth Edition. 12mo., cloth,

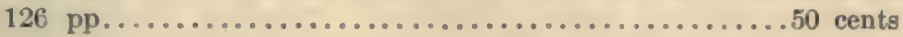

BOMANS, J. E. A B C of the Telephone. With 269 Illustrations.

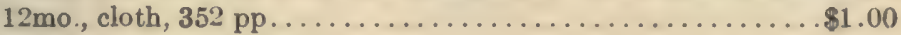

HOPKINS, N. M. Experimental Electrochemistry, Theoretically and Practically Treated. Profusely illustrated with 130 new drawings, diagrams, and photographs, accompanied by a Bibliography. Illustrated. 8vo, cloth, 299 pp............................. $\$ 3.00$

HOUSTON, EDWIN J. A Dictionary of Electrical Words, Terms, and Phrases. Fourth Edition, rewritten and greatly enlarged. 582 Illus. trations. 4to., cloth........................... $\$ 7.00$

-A Pocket Dictionary of Electrical Words, Terms, and Phrases, $12 \mathrm{mo.}$, cloth, 950 pp................................... $\$ 2.50$

HOUSTOUN, R. A. Studies in Light Production. 22 illustrations.

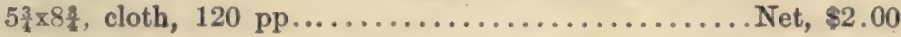

HUTCHINSON, R. W., Jr. Long-Distance Electric Power Transmission: Being a Treatise on the Hydro-Electric Generation of Energy; Its Transformation, Transmission, and Distribution. Second Edition. Illustrated. $12 \mathrm{mo}$., eloth, $350 \mathrm{pp} . \ldots \ldots \ldots \ldots \ldots \ldots$. Net, $\$ 3.00$ and IHLSENG, M. C. Electricity in Mining. Being a theoretical and practical treatise on the construction, operation and maintenance of electrical mining machinery. 12mo.; cloth...... In Press

INCANDESCENT ELECTRIC LIGHTING. A Practical Description of the Edison System, by H. Latimer. To which is added: The Design and Operation of Incandescent Stations, by C. J. Field; A Description of the Edison Electrolyte Meter, by A. E. Kennelly; and a Paper on the Maximum Efficiency of Incandescent Lamps, by T. W. Howell. Fifth Edition. Illustrated. 16mo., cloth, 140 pp. (No. 57 Van Nostrand's Science Series.).............50 cents

INDUCTION COILS: How Made and How Used. Eleventh Edition. Illustrated. 16mo., cloth, 123 pp. (No. 53 Van Nostrand's Seience

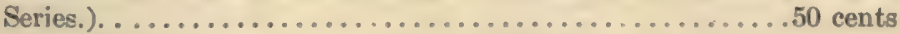

JEHL, FRANCIS. The Manufacture of Carbons for Electric Lighting and other purposes. Illustrated with numerous Diagrams, Tables, and Folding Plates. 8vo., cloth, 232 pp.................. $\$ 4.00$

JOHNSON, J. H. Arc Lamps and Accessory Apparatus. 20 illustrations. $16 \mathrm{mo}$., cloth, $135 \mathrm{pp}$. (Installation Manuals Series). Net, 75 cents. 
JOHNSON, T. M. Ship Wiring and Fitting. 47 illustrations. 16imo, cloth, $92 \mathrm{pp}$. (Installation Manuals Series)......... Net, 75 cents.

JONES, HARRY C. The Electrical Nature of Matter and Radioactivity. Second Edition, revised and enlarged. 12mo., cloth, $218 \mathrm{pp} . \$ 2.00$

KAPP, GISBERT. Electrical Transmission of Energy and its Transformation, Subdivision, and Distribution. A Practical Handbook. Fourth Edition, thoroughly revised. Illustrated. $12 \mathrm{mo}$., cloth, $445 \mathrm{pp} . . \$ 3.50$

Alternate-Current Machinery. Illustrated. $16 \mathrm{mo}$, cloth, $190 \mathrm{pp}$. (No. 96 Van Nostrand's Science Series.). . . . . . . . . . . 50 cents

KEMPE, H. R. A Handbook of Electrical Testing. Seventh Edition, revised and enlarged. Illustrated. 8vo., cloth, 706 pp... Net, \$6.00

KENNEDY, R. Electrical Installations of Electric Light, Power, and Traction Machinery. Illustrated. 8vo, cloth, 5 vols. The set $\$ 15.00$. Each .................................... $\$ 30$

KENNELLY, A. E. Theoretical Elements of Electro-Dynamic Machinery. Vol. I. Illustrated. 8vo., cloth, $90 \mathrm{pp} \ldots \ldots \ldots \ldots \ldots \ldots \ldots 1.50$

KERSHAW, J. B. C. The Electric Furnace in Iron and Steel Production. Illustrated. 8vo., cloth, 74 pp..................... $\$ 1.50$ Electrometallurgy. Illustrated. 8vo., cloth, 303 pp. (Van Nostrand's Westminster Series.)..................... $\$ 2.00$

KINZBRUNNER, C. Continuous-Current Armatures; their Winding and Construction. 79 Illustrations. 8 vo., cloth, $80 \mathrm{pp} \ldots .$. Net, $\$ 1.50$ Alternate-Current Windings; their Theory and Construction. 89 Illustrations. 8vo.; cloth, $80 \mathrm{pp} \ldots \ldots \ldots \ldots \ldots \ldots \ldots$........... $\$ 1.50$

KOESTER, F. Hydroelectric Developments and Engineering. A practical and theoretical treatise on the development, design, construction, equipment and operation of hydroelectric transmission plants. Second Edition. 500 illustrations. 4to., cloth, $475 \mathrm{pp}$... Net, $\$ 5.00$

- Steam-Electric Power Plants. A practical treatise on the design of central light and power stations and their economical construction and operation. Second Edition. Fully Illustrated. 4to., eloth, 455 pp..................................... $\$ 5.00$

LARNER, E.T. The Principles of Alternating Currents for Students of Electrical Engineering. Illustrated with Diagrams. 12mo., cloth, 144 pp......................................... $\$ 1.25$ 
LEMSTROM, S. Electricity in Agriculture and Horticulture. Illustrated. 8 vo., eloth................................. $\$ 1.50$

LIVERMORE, V. P., and WILliAMS, J. How to Become a Competent Motorman: Being a practical treatise on the proper method of operating a street-railway motor-car; also giving details how to overcome certain defects. Second Edition. Illustrater. 16mo., eloth,

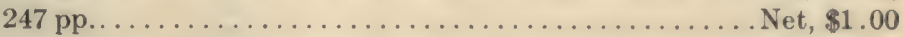

LOCKWOOD, T. D. Electricity, Magnetism, and Electro-Telegraphy. A Practical Guide and Handbook of General Information for Electrical Students, Operators, and Inspectors. Fourth Edition. Illustrated. 8vo., cloth, 374 po..................\$2.50

LODGE, OLIVER J. Signalling Across Space Without Wires: Being a description of the work of Hertz and his successors. Third Edition. Illustrated. 8 vo., eloth............................. $\$ 2.00$

LORING, A. E. A Handbook of the Electro-Magnetic Telegraph Fourth Edition, revised. Illustrated. 16mo., cloth, $116 \mathrm{pp}$. (No. 39 Van Nostrand's Science Series.). . . . . . . . . . . 50 cents

LUPTON, A., PARR, G. D. A., and PERKIN, H. Electricity Applied to Mining. Second Edition. With Tahles, Diagrams, and Folding Plates. 8vo., cloth, $320 \mathrm{pp} \ldots \ldots \ldots \ldots \ldots \ldots \ldots$.............. $\$ 4.50$

MANSFIELD, A. N. Electromagnets : Their Design and Construction. Second Edition. Illustrated. 16mo., cloth, 155 pp. (No.64 Van Nostrand's Science Series.)...................50 cents

MASSIE, W. W., and UNDERHILL, C. R. Wireless Telegraphy and Telephony Popularly Explained. With a chapter by Nikola Tesla. Illustrated. 12mo., cloth, $82 \mathrm{pp} . \ldots \ldots \ldots \ldots \ldots$............ $\$ 1.00$

MAURICE, W. Electrical Blasting Apparatus and Explosives, with special reference to colliery practice. Illustrated. 8vo, clith.

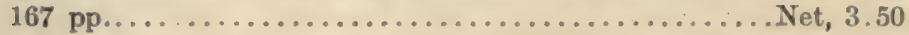

- The Shot Firer's Guide. A practical manual on blasting and the prevention of blasting accidents. 78 illustrations. 8vo., cloth.

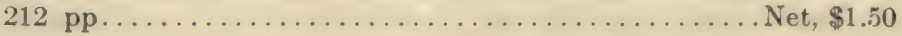

MONCKTON, C. C. F. Radio Telegraphy. 173 Illustrations. 8vo. cloth, 272 pp. (Van Nostrand's Westminster Series.).... Net, \$2.0n

MORECROFT, J. H. and HEHRE, F. W. A Short Course in Testing of Electrical Machinery. Second Edition. Illustrated. 8vo., cloth,

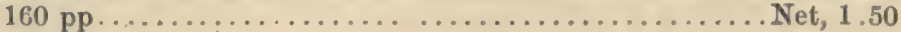


MORGAN, ALFRED P. Wireless Telegraph Construction for Amateurs. 153 illustrations. $12 \mathrm{mo}$, eloth, $220 \mathrm{pp} . \ldots \ldots \ldots \ldots \ldots$. Net, $\$ 1.50$

NIPHER, FRANCIS E. Theory of Magnetic Measurements. With an Appendix on the Method of Least Squares. Illustrated. 12mo.,

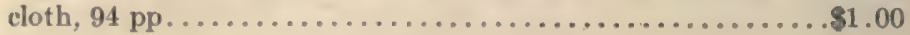

OHM, G. S. The Galvanic Circuit Investigated Mathematically. Berlin, 1827. Translated by William Francis. With Preface and Notes by Thos. D. Lockwood. Second Edition. Illustrated. $16 \mathrm{mo}$., cloth, 269 pp. (No. 102 Van Nostrand's Ścience Series.)........50 cents

OLSSON, ANDREW. Motor Control as used in Connection with Turret Turning and Gun Elevating. (The Ward Leonard System.) 13 illustrations. 12mo., paper, 27 pp. (U. S. Navy Electrical Series No. 1.)..................................... . 50

OUDIN, MAURICE A. Standard Polyphase Apparatus and Systems. Fifth Edition, revised. Illustrated with many Photo-reproductions, Diagrams, and Tables. 8 vo.. oloth, 369 pp........... Net, \$3.00

PALAZ, A. Treatise on Industrial Photometry. Specially applied to Electric Lighting. Translated from the French by G. W. Patterson, Jr., and M. R. Patterson. Second Edition. Fully Illustrated. 8 vo., clnth, 324 pp.........................\$4.00

PARR, G. D. A. Electrical Engineering Measuring Instruments for Commercial and Laboratory Purposes. With 370 Diagrams and Engravings. 8vo., cloth, $328 \mathrm{pp} . . \ldots \ldots \ldots \ldots \ldots \ldots \ldots$. Net, $\$ 3.50$

PARSHALL, H. F., and HOBART, H. M. Armature Windings of Electric Machines. Third Edition. With 140 full-page Plates, 65 Tables, and 16.5 pages of descriptive letter-press. 4 to., cloth, $300 \mathrm{pp} . \$ 7.50$ Electric Railway Engineering. With 437 Figures and Diagrams and many Tables. 4to., cloth, $475 \mathrm{pp} \ldots \ldots \ldots \ldots$ Net, $\$ 10.00$

Electric Machine Design. Being a revised and enlarged edition of "Electric Generators." 648 Illustrations. 4to., half morocco, 601 pp.................................... \$12.50

PERRINE, F. A. C. Conductors for Electrical Distribution: Their Manufacture and Materials, the Calculation of Circuits, Pole-Line Construction, Underground Working, and other Uses. Second Edition. Illustrated. 8vo., cloth, 287 pp.............. Net, $\$ 3.50$ 
POPE, F. L. Modern Practice of the Electric Telegraph. A Handbook for Electricians and Operators. Seventeenth Edition. Illustrated. 8 vo., cloth, 234 pp ..........................51.50

RAPHAEL, F. C. Localization of Faults in Electric Light Mains. Second Edition, revised. Illustrated. Svo., cloth, $205 \mathrm{pr} \ldots . .$. Net, $\$ 3.00$

RAS $2 H$, E. Electric Arc Phenomena. Illustrated by K. Tirnberg. 汭 illustratons. 12mo., eloth, $200 \mathrm{pp} . . \ldots \ldots \ldots \ldots \ldots$. In Press

RAYMOND, E. B. Alternating-Current Engineering, Practically Treated. Third Edition, revised. With many Figures and Diagrams. 8vo., cloth, 244 pp.............................. \$2.50

RICHARDSON, S.S. Magnetism and Electricity and the Principles of Elec trical Measurement. Illustrated. 12mo., cloth, $596 \mathrm{pp}$. . Net, $\$ 2.00$

ROBERTS, J. Laboratory Work in Electrical Engineering-Preliminary Grade. A series of laboratory experiments for first and secondyear students in electrical engineering. Illustrated with many Diagrams. 8vo., cloth, 218 pp................... $\$ 2.00$

ROLLINS, W. Notes on X-Light. Printed on deckle edge Japan paper $4(1) \mathrm{pp}$. of text, 152 full-page plates. $8 \mathrm{vo}$., cloth. ....... Net, $\$ 5.00$

RUHMER, ERNST. Wireless Telephony in Theory and Practice. Translated from the German by James Erskine-Murray. Illustrated. $8 v o$. , cloth, 224 pp.............................. $\$ 3.50$

RUSSELL, A. The Theory of Electric Cables and Networks. 71 Illustrations. 8vo., eloth, $275 \mathrm{pp} . \ldots \ldots \ldots \ldots \ldots \ldots$............... $\$ 3.00$

SALOMONS, DAVID. Electric-Light Installations. A Practical Handbook. Illustrated. $12 \mathrm{mo}$., eloth.

Vol. I.: Management of Accumulators. Vinth Edition. $178 \mathrm{pp} . \$ 2.50$ Vol. II.: Apparatus. Seventh Edition. 318 pp..........\$2.25 Vol III.: Application. Seventh Edition. $234 \mathrm{pp} \ldots \ldots \ldots \ldots \ldots 1.50$

SEVER, G. F. Electrical Engineering Experiments and Tests on DirectCurrent Machinery. Second Edition, enlarged. With Diagrams and Figures. 8vo., pamphlet, $75 \mathrm{pp} . \ldots \ldots \ldots \ldots \ldots \ldots$.......... $\$ 1.00$

SEVER, G. F., and TOWNSEND, F. Laboratory and Factory Tests in Electrical Engineering. Second Edition, revised and enlarged. Illustrated. 8 vo., eloth, $269 \mathrm{pp} . . \ldots \ldots \ldots \ldots \ldots$............... $\$ 2.50$ 
SEWALL, C. H. Wireless Telegraphy. With Diagrams and Figures. Second Edition, corrected. Illustrated. 8vo., cloth, 229 pp... Net. \$2.00

_Lessons in Telegraphy. Illustrated. $12 \mathrm{mo}$., cloth, $104 \mathrm{pp}$. Net, $\$ 1.00$

SEWELL, T. Elements of Electrical Engineering. Third Edition, revised. Illustrated. 8vo., cloth, $444 \mathrm{pp} \ldots \ldots \ldots \ldots \ldots \ldots 3.00$

- The Construction of Dynamos (Alternating and Direct Current). A Text-book for students, engineering contractors, and electricians-incharge. Illustrate!. 8vi., cloth, 316 pp............ \$3.00

SHAW, P. E. A First-Year Course of Practical Magnetism and Electricity. Specially arlapted to the wants of technical students. Illustrated. 8vo., cloth, $66 \mathrm{pp}$. interleaved for note taking. . . . . . . Net, $\$ 1.00$

SHELDON, S., and HAUSMANN, E. Dynamo-Electric Machinery: Its Construction, Design, and Operation.

Vol. I.: Direct-Current Machines. Eighth Edition, completely re-written. Illustrated. $12 \mathrm{mo}$., cloth, $281 \mathrm{pp} \ldots \ldots \ldots \ldots \ldots \ldots$. Net, $\$ 2.50$

Vol. II.: Alternating-Current Machines: Eighth Edition, rewritten. cloth, 353 pp............................... $\$ 2.50$

- Electric Traction and Transmission Engineering 127 illustration. $12 \mathrm{mo}$., cloth, 317 pp........................... $\$ 2.50$

SLOANE, T. O'CONOR. Standard Electrical Dictionary. 300 Illustrations. $12 \mathrm{mo}$., eloth, 682 pp...................\$3.00

- Elementary Electrical Calculations. A Manual of Simple Engineering Mathematics, covering the whole field of Direct Current Calculations, the basis of Alternating Current Mathematics, Networks, and typical cases of Circuits, with Appendices on special subjects. 8vo., cloth. Illustrated. $304 \mathrm{pp} \ldots \ldots \ldots$. Net, $\$ 2.00$

SNELL, ALBION T. Electric Motive Power. The Transmission and Distribution of Flectric Power by Continuous and Alternating Currents. With a Section on the Applications of Electricity to Mining Work. Second Edition. Illustrated. 8vo., cloth, $411 \mathrm{pp} \ldots \ldots .$. Net, $\$ 4.00$

SODDY, F. Radio-Activity; an Elementary Treatise from the Standpoint of the Disintegration Theory. Fully Illustrated. 8vo., cloth,

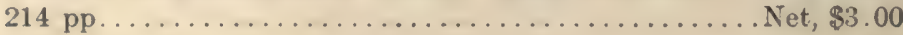

SOLOMON, MAURICE. Electric Lamps. Illustrated. 8vo., cloth. (Van Nostrand's Westminster Series.)................ Net, $\$ 2.00$ 
STEWART, A. Modern Polyphase Machinery. Illustrated. $12 \mathrm{mo.}$ cloth, 296 pp............................. $\$ 2.00$

SWINBURNE, JAS., and WORDINGHAM, C. H. The Measurement of Flectric Currents. Electrical Measuring Instruments. Meters for Electrical Energy. Eslited, with Preface, by T. Commerford Martin, Folding Plate and Numerous Illustrations. 16mo, cloth, $241 \mathrm{pp}$. (No. 109 Van Nostrand's Science Series.)...........50 cents

SWOOPE, C. WALTON. Lessons in Practical Electricity: Principle Experiments, and Arithmetical Problems. An Elementary Texts book. With numerous Tables, Formulæ, and two large Instruction Plates. Twelfth Edition, revised. Illustrated. 8vo., cloth, $462 \mathrm{pp}$.

Net, $\$ 2.00$

THIESS, J. B. and JOY, G. A. Toll Telephone Practice. 268 illustrations 8 vo., eloth, 429 pp........................... $\$ 3.50$

THOM, C., and JONES, W. H. Telegraphic Connections, embracing recent methods in Quadruplex Telegraphy. 20 Colored Plates. 8vo.,

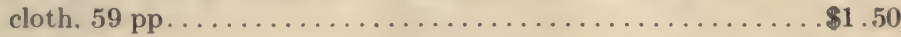

THOMPSON, S. P. Dynamo-Electric Machinery. With an Introduction and Notes by Frank I. Pope and H. R. Butler. Fully Illustrated. 16mo., eloth, 214 pp. (No. 66 Van Nostrand's Seience Series.)

50 cents

- Recent Progress in Dynamo-Electric Machines. Being a Supplement to "Dynamo-Electric Machinery." Illustrated. 16mo., cloth, $113 \mathrm{pp}$. (No. 75 Van Nostrand's Science Series.)...........50 cent.

TOWNSEND, FITZHUGH. Alternating Current Engineering. Illustrated. 8 vo., paper, $32 \mathrm{pp} . . . \ldots \ldots \ldots \ldots \ldots \ldots$. Net, 75 cents

UNDERHILL, C. R. Solenoids, Electromagnets and Electromagnetic Windings. 218 Illustrations. $12 \mathrm{mo}$., cloth, $345 \mathrm{pp} . .$. . Net, $\$ 2.00$

URQUHART, J. W. Electroplating. A Practical Handbook. Fifth Edition. Illustrated. 12mo., cloth, $230 \mathrm{pp} \ldots \ldots \ldots \ldots \ldots \ldots 2.00$

—-Electrotyping. Illustrated. 12mo., cloth, 228 pp.......\$2.00

WADE, E. J. Secondary Batteries: Their Theory, Construction, and Use. Second Edition, corrected. 265 Illustrations, 8vo., cloth, 501 pp. Net, \$4.00 
WADSWORTH, C. Primary Bcttery Ignition. A simple practical pocket guide on the construction, operation, maintenance, and testing of primary batteries for automobile, motorboat, and stationary. engine ignition service. 26 illustrations. $5 \times 7$, cloth, $79 \mathrm{pp}$. Net.0.50

WALKER, FREDERICK. Practical Dynamo-Building for Amateurs. How to Wind for any Output. Third Edition. Illustrated. 1 (imo., cloth, 104 pn. (No. 98 Van Nostrand's Science Series.)....50 cents

Electricity in Mining. Illustrated, 8vo., eloth, $385 \mathrm{pp} \ldots \ldots . \$ 3.50$

WALLING, B. 'T., and MARTIN, JULIUS. Electrical Installations of the United States Navy. With many Diagrams and Engravings. Svo., cloth, 648 pp........................... \$6.00

WATT, ALEXANDER. Electroplating and Refining of Metals. lew Edition, rewritten by Arnold Philip. Illustrated. 8vo., cloth, 677 pp......................................... $\$ 4.50$

—Electro-metallurgy. Fifteenth Edition. Illustrated. 12mo, cloth, 225

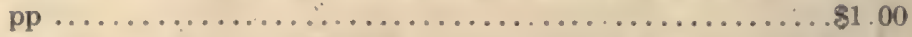

WEBB, H. L. A Practical Guide to the Testing of Insulated Wires and Cables. Fifth Edition. Illustrated. 12mo., cloth, 118 pp...\$1.00

WEEKS, R. W. The Design of Alternate-Current Transformer.

New.Edition in Press

WEYMOUTH, F. MARTEN. Drum Armatures and Commutators. (Theory and Practice.) A complete treatise on the theory and construction of drum-winding, and of commutators for closed-coil armatures, together with a full résumé of some of the principal points involved in their design, and an exposition of armature reactions and sparking. Illustrated. 8vo., cloth, $295 \mathrm{pp} \ldots \ldots \ldots$.... Net, $\$ 3.00$

WILKINSON, H. D. Submarine Cable Laying, Repairing and Testing. Second Edition, completely revised. 313 Illustrations. Svo., eloth,

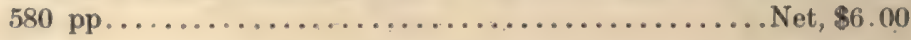

WRIGHT, J. Testing, Fault Localization and General Hints for Linemen. 19 illustrations. $16 \mathrm{mo}$., cloth, $88 \mathrm{pp}$. (Installation Series Manuals.) Net, 50 cents. 
YOUNG, J. ELTON. Electrical Testing for Telegraph Engineers. Illustrated. $8 v o$., cloth, 264 pp........................ $\$ 4.00$

ZEIDLER, J., and LUSTGARTEN, J. Electric Arc Lamps: Their Principles, Construction and Working. 160 Illustrations. 8vo., cloth, 188 p..................................... $\$ 2.00$

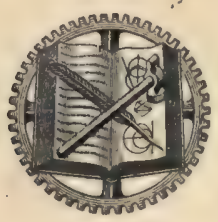

A 96-page Catalog of Books on Electricity, classified by subjects, will be furnished gratis, postage prepaid, on application. 


\section{VAn Nostrand Company}

are prepared to supply, either from their complete stock or at short notice,

Any Technical or

\section{Scientific Book}

In addition to publishing a very large and varied number of SCIENTIFIC AND Engineering Books, D. Van Nostrand Company have on hand the largest assortment in the United States of such books issued by American and foreign publishers.

All inquiries are cheerfully and carefully answered and complete catalogs sent free on request.

25 Park Place. - . . . . New York 



\section{PLEASE DO NOT REMOVE \\ CARDS OR SLIPS FROM THIS POCKET}

\section{UNIVERSITY OF TORONTO LIBRARY}

S\&M

A

174 
